ANALYSIS \& PDE

Volume $4 \quad$ No. $5 \quad 2011$

\title{
STANDING RING BLOWUP SOLUTIONS FOR CUBIC NONLINEAR SCHRÖDINGER EQUATIONS
}

$$
\text { IAN ZWIERS }
$$




\title{
STANDING RING BLOWUP SOLUTIONS FOR CUBIC NONLINEAR SCHRÖDINGER EQUATIONS
}

\author{
IAN ZWIERS
}

For all dimensions $N \geq 3$ we prove there exist solutions to the focusing cubic nonlinear Schrödinger equations that blow up on a set of codimension two. The blowup set is identified both as the site of $L^{2}$ concentration and by a bounded supercritical norm outside any neighborhood of the set. In all cases, the global $H^{1}$ norm grows at the log-log rate.

\section{Introduction}

Consider the cubic focusing nonlinear Schrödinger equation in dimension $N \geq 3$ :

$$
\left\{\begin{array}{l}
i u_{t}+\Delta u+u|u|^{2}=0 \\
u(0, x)=u_{0}: \mathbb{R}^{N} \rightarrow \mathbb{C}
\end{array}\right.
$$

This is a canonical model equation arising in physics and engineering [Sulem and Sulem 1999]. This equation, and other closely related equations, have been the subject of many recent mathematical studies.

Equation (1-1) is locally wellposed for data

$$
u_{0} \in H^{s}\left(\mathbb{R}^{N}\right)
$$

for any $s \in\left(\frac{N}{2}-1, \frac{N}{2}\right.$ ] or integer $s>\frac{N}{2}$; see [Cazenave 2003]. In these cases we have the classic blowup alternative: either $T_{\max }=+\infty$ or $\|u(t)\|_{H^{s}} \rightarrow \infty$ as $t \rightarrow T_{\max }$. Higher regularity persists under local-in-time dynamics and the maximal time $T_{\max }>0$ for which $u$ belongs to $C\left(\left[0, T_{\max }\right), H^{s}\right)$ is the same for all $s>\frac{N}{2}-1$. Evolution under (1-1) preserves

$$
\begin{aligned}
& \int_{\mathbb{R}^{3}}|u(t, x)|^{2} d x=\int\left|u_{0}\right|^{2} d x=M\left[u_{0}\right] \\
& \int\left|\nabla_{x} u(t, x)\right|^{2} d x-\frac{1}{2} \int|u(t, x)|^{4} d x=E[u(t, x)]=E\left[u_{0}\right] \\
& \operatorname{Im}\left(\int \bar{u}(t, x) \nabla u(t, x) d x\right)=\operatorname{Im}\left(\int \overline{u_{0}} \nabla u_{0} d x\right)
\end{aligned}
$$

MSC2000: primary 35Q55; secondary 35B40.

Keywords: focusing, nonlinear Schrödinger equation, supercritical, collapse, blowup rate, blowup set, codimension, regularity, $\log -\log$ rate. 
There are corresponding symmetries. If $u(t, x)$ satisfies (1-1), so do the following:

$$
\begin{array}{rll}
u\left(t, x+x_{0}\right) & \forall x_{0} \in \mathbb{R}^{N} & \text { (spatial translation invariance) } \\
u\left(t+t_{0}, x\right) & \forall t_{0} \in \mathbb{R} & \text { (time translation invariance) } \\
u(t, x) e^{i \gamma_{0}} & \forall \gamma_{0} \in \mathbb{R} & \text { (phase invariance) } \\
u\left(t, x-\beta_{0} t\right) e^{i \frac{\beta_{0}}{2} \cdot\left(x-\frac{\beta_{0}}{2} t\right)} & \forall \beta_{0} \in \mathbb{R}^{N} & \text { (Galilean invariance) } \\
\lambda_{0} u\left(\lambda_{0}^{2} t, \lambda_{0} x\right) & \forall \lambda_{0}>0 & \text { (scaling invariance) }
\end{array}
$$

Scaling invariance leaves the $\dot{H}^{\frac{N}{2}-1}\left(\mathbb{R}^{N}\right)$ norm of data unchanged and for this reason Equation (1-1) is deemed $H^{\frac{N}{2}-1}$-critical. Local wellposedness for $s>\frac{N}{2}-1$ and the scaling symmetry prove that all solutions that blow up in finite time $T_{\max }<+\infty$ must obey the scaling lower bounds

$$
\|u(t)\|_{H^{s}} \gtrsim \frac{1}{\left(T_{\max }-t\right)^{\frac{s}{2}-\frac{N-2}{4}}} .
$$

Equation (1-1) has standing wave solutions. The ansatz $u(t, x)=e^{i t} W(x)$ leads to the elliptic PDE

$$
\left\{\begin{array}{l}
\Delta W-W+W|W|^{2}=0 \\
W(|x|)>0 \text { for } x \in \mathbb{R}^{N}
\end{array}\right.
$$

The unique positive radial solution ${ }^{1}$ to (1-5) is the ground-state solution of (1-1). We reserve the notation $Q$ for the ground-state solution of the two-dimensional problem,

$$
\left\{\begin{array}{l}
\Delta_{\mathbb{R}^{2}} Q-Q+Q|Q|^{2}=0 \\
Q(|y|)>0 \text { for } y \in \mathbb{R}^{2}
\end{array}\right.
$$

Classification of dynamics. In the case $N=2$, if $M\left[u_{0}\right]<M[Q]$ solutions to (1-1) exist for all time [Weinstein 1983] and scatter [Killip et al. 2009]. Negative-energy data $u_{0} \in H^{1}$ lead to blowup in finite time if it is radially symmetric or has finite variance, $u_{0} \in \Sigma=H^{1} \cap\left\{f:|x| f(x) \in L^{2}\right\}$; see [Ogawa and Tsutsumi 1991]. By adjusting the quadratic phase of negative-energy data, one can produce examples of blowup solutions with arbitrary energy [Cazenave 2003, Remark 6.5.9]. ${ }^{2}$ At the threshold $M\left[u_{0}\right]=M[Q]$ there is, up to symmetries, a unique explicit blowup solution [Merle 1993].

In the cases $N=3$ and $N=4$ the situation is more complicated. Assume that

$$
M\left[u_{0}\right]^{4-N} E\left[u_{0}\right]^{N-2}<M[W]^{4-N} E[W]^{N-2} .
$$

The following classification is independent of time:

$$
\begin{array}{ll}
\|u(t)\|_{L^{2}}^{4-N}\|\nabla u(t)\|_{L^{2}}^{N-2}<\|W\|_{L^{2}}^{4-N}\|\nabla W\|_{L^{2}}^{N-2}, & \text { with global existence and scattering, or } \\
\|u(t)\|_{L^{2}}^{4-N}\|\nabla u(t)\|_{L^{2}}^{N-2}>\|W\|_{L^{2}}^{4-N}\|\nabla W\|_{L^{2}}^{N-2}, & \text { with unbounded } H^{1} \text { norm growth. }
\end{array}
$$

\footnotetext{
${ }^{1}$ The classic proof in the case $N=3$ is in [Coffman 1972]. For other dimensions, see [Weinstein 1983; Berestycki and Lions 1983; Kwong 1989]. For a concise overview of these results, see [Tao 2006, Appendix B].

${ }^{2}$ These arguments also apply to the other energy-subcritical case, $N=3$. Further sufficient conditions for blowup based on the virial identity in $N=3$ are known; see [Holmer et al. 2010].
} 
This was shown in [Kenig and Merle 2006] in the case $N=4$ with radial symmetry and in [Duyckaerts et al. 2008; Holmer and Roudenko 2008; 2010] in the case $N=3$. Again, the data at the threshold of (1-7) may be classified in terms of three special solutions, up to symmetries; see [Duyckaerts and Merle 2009; Duyckaerts and Roudenko 2010]. One of these solutions blows up in finite time, but the exact dynamic is unknown.

In all cases, the description of behavior above the threshold is an ongoing challenge.

In the case of $N=2$, Merle and Raphäl have completely described the dynamic of an open class of data with $M[Q]<M\left[u_{0}\right]<M[Q]+\delta$. This open class includes all data with negative energy and is thought to describe the generic behavior. See Theorem 1.1, below, for references. Their work follows the earlier simulation [Landman et al. 1988] and construction [Perelman 2001] of solutions in $H^{1}$ that blow up at the rate of the scaling lower bound with a log-log correction.

Recently, in the case of $N=3$, data with $M[W] E[W]<M\left[u_{0}\right] E\left[u_{0}\right]<M[W] E[W]+\delta$ has been shown in [Nakanishi and Schlag 2010] to satisfy one of nine scenarios involving scattering, finite-time blowup, or trapping in the neighborhood of a manifold of solitons.

Known blowup regimes. There remain, in all cases, very few examples of explicit blowup regimes. We have already alluded to Merle and Raphaël's results in the case $N=2$ :

Theorem 1.1 (log-log blowup of $L^{2}$-critical NLS [Merle and Raphaël 2003; 2004; 2005a; 2006; Raphaël 2005]). Consider the focusing $L^{2}$-critical NLS in dimension $1 \leq d \leq 5$,

$$
i u_{t}+\Delta u+u|u|^{\frac{4}{d}}=0 .
$$

There exists an open set of data in $H^{1}\left(\mathbb{R}^{d}\right)$, with mass a little larger than the groundstate, that blow up at the log-log rate:

$$
\|u(t)\|_{H^{1}} \approx\left(\frac{\log \left|\log \left(T_{\max }-t\right)\right|}{T_{\max }-t}\right)^{\frac{1}{2}} .
$$

These solutions concentrate exactly the groundstate profile in $L^{2}$ at a point. That is, the remainder of the solution has a strong limit in $L^{2}$ as $t \rightarrow T_{\max }$. Moreover, data in the same range of mass that do not belong to the log-log class give solutions that either exist for all time, or blow up at the rate $\|u(t)\|_{H^{1}} \gtrsim\left(T_{\max }-t\right)^{-1}$.

Theorem 1.1 gives a precise understanding of the stable blowup regime of the one-dimensional quintic NLS. To prove the next theorem, Raphaël demonstrated a reduction of the two-dimensional quintic problem (which is $H^{\frac{1}{2}}$-critical) to this one-dimensional log-log regime.

Theorem 1.2 (standing ring blowups for quintic NLS in 2D [Raphaël 2006]). There exists an open set of radially symmetric data in $H^{1}\left(\mathbb{R}^{2}\right)$ for which the corresponding solution to i $u_{t}+\Delta u+u|u|^{4}=0$ exhibits blowup at the log-log rate, (1-8), and concentration in $L^{2}$ at a ring of fixed radius.

The argument was extended to reduce the energy critical and supercritical quintic equations to the same one-dimensional log-log regime: 
Theorem 1.3 (codimension-one blowups for quintic NLS [Raphaël and Szeftel 2009]). For all $N \geq 3$, there exists an open set of radially symmetric data in $H^{N}\left(\mathbb{R}^{N}\right)$ for which the corresponding solution to $i u_{t}+\Delta u+u|u|^{4}=0$ exhibits blowup at the log-log rate and concentration in $L^{2}$ at a fixed radius.

Our aim is to implement this approach for the cubic problem, and, under cylindrical symmetry, reduce the $N$-dimensional problem (1-1) to the two-dimensional problem, which we understand by Theorem 1.1. The following result and our main result, Theorem 1.6, were developed simultaneously. ${ }^{3}$

Theorem 1.4 (standing ring blowups for cubic NLS in 3D [Holmer and Roudenko 2011]). There exists an open set of cylindrically symmetric data in $H^{1}\left(\mathbb{R}^{3}\right)$ for which the corresponding solution to (1-1) exhibits blowup at the log-log rate and concentration in $L^{2}$ at a ring of fixed radius.

The stability proven in Theorem 1.4 is at the level of $H^{1}$ regularity, which allows for a different approach and a larger class of data than our main result in the case $N=3$. The new techniques of Theorem 1.4 are not directly applicable for the energy critical and supercritical cases, $N>3$.

It is anticipated that the $L^{2}$-supercritical NLS will demonstrate further unique blowup behavior not described by Theorems 1.2, 1.3, 1.4 or our Theorem 1.6. In particular, due to the asymptotic analysis of Fibich, Gavish, and Wang [Fibich et al. 2007], all supercritical problems with subquintic nonlinearities are expected to admit radially symmetric blowup solutions that focus onto a shell whose radius collapses to zero. In the case of cubic nonlinearity and dimension $N$, the shell is expected to have radius $\approx$ $\left(T_{\max }-t\right)^{N /(N-1)^{2}}$. In the case $N=3$, the $H^{1}$ norm is expected to grow at the scaling lower bound. See also [Holmer and Roudenko 2007]. The existence and uniqueness of these radial blowup solutions remain important problems.

Notation 1.5. We use $f \lesssim g, f \gtrsim g$ and $f \approx g$ to denote that there exist constants $C_{1}, C_{2}>0$ such that $f \leq C_{1} g, f \geq C_{2} g$, and $C_{2} g \leq f \leq C_{1} g$, respectively. The notation $f \sim g$ is used in more casual discussion to say that $f$ and $g$ are of the same order. We will use $\delta(\alpha)$ to denote any function of $\alpha$ with the property $\delta(\alpha) \rightarrow 0$ as $\alpha \rightarrow 0$. The exact form of $\delta$ will depend on the context. Frequently, we use the operator

$$
\Lambda=1+y \cdot \nabla_{y}, \quad \text { where } y \text { is a two-dimensional variable. }
$$

For $f, g \in L^{2}\left(\mathbb{R}^{2}\right)$ we have $(\Lambda f, g)=-(f, \Lambda g)$.

Statement of result. For all $N \geq 3$ we introduce cylindrical coordinates $x=(r, z, \theta) \in[0, \infty) \times \mathbb{R} \times S^{N-2}$ for $x \in \mathbb{R}^{N}$. We refer to functions that are symmetric with respect to $\theta$ as cylindrically symmetric, and we let $H_{\text {cyl }}^{s}\left(\mathbb{R}^{N}\right)$ denote the cylindrically symmetric subset of $H^{s}$.

Theorem 1.6 (main result). For all $N \geq 3$, there exists a set of cylindrically symmetric data $u_{0} \in \mathscr{P}$, open in $H_{\mathrm{cyl}}^{N}\left(\mathbb{R}^{N}\right)$, for which the corresponding solution $u(t)$ of (1-1) has maximum (forward) lifetime $0<T_{\max }<+\infty$ and exhibits the following properties:

\footnotetext{
${ }^{3}$ After Theorem 1.2, the idea of considering other $H^{\frac{1}{2}}$-critical problems was first suggested to the author's thesis advisor by Justin Holmer and Svetlana Roudenko in private conversation.
} 
Concentration. There exist parameters $\lambda(t)>0, r(t)>0, z(t) \in \mathbb{R}^{N-2}$, and $\gamma(t) \in \mathbb{R}$, with convergence

$$
(r(t), z(t)) \longrightarrow\left(r_{\max }, z_{\max }\right) \quad \text { as } t \rightarrow T_{\max }, \text { with } r_{\max } \sim 1,
$$

such that there is the following strong convergence in $L^{2}\left(\mathbb{R}^{N}\right)$ :

$$
u(t, r, z, \theta)-\frac{1}{\lambda(t)} Q\left(\frac{(r, z)-(r(t), z(t))}{\lambda(t)}\right) e^{-i \gamma(t)} \longrightarrow u^{*}(r, z, \theta) \quad \text { as } t \rightarrow T_{\max } .
$$

Persistent regularity away from singular ring. For any $R>0$,

$$
u^{*} \in H^{\frac{N}{2}-\frac{1}{2}}\left(\left|(r, z)-\left(r_{\max }, z_{\max }\right)\right|>R\right) .
$$

Log-log blowup rate. The solution leaves $H^{1}$ at the log-log rate:

$$
\frac{\left(\frac{\log \left|\log T_{\max }-t\right|}{T_{\max }-t}\right)^{\frac{1}{2}}}{\|u(t)\|_{H^{1}\left(\mathbb{R}^{3}\right)}} \longrightarrow \frac{\sqrt{2 \pi}}{\|Q\|_{L^{2}\left(\mathbb{R}^{2}\right)}} \text { as } t \rightarrow T_{\max } .
$$

Moreover, the higher-order norm behaves appropriately:

$$
\frac{\|u(t)\|_{H^{N}}}{\|u(t)\|_{H^{1}}^{N} \log \|u(t)\|_{H^{1}}} \longrightarrow 0 \quad \text { as } t \rightarrow T_{\max } .
$$

Remark 1.7 (nature of $u^{*}$ ). For the $L^{2}$-critical problem, Theorem 1.1, it is known that the residual profile $u^{*}$ is not in $H^{1}$ [Merle and Raphaël 2005b]. Indeed, (1-11) fails for $R=0$. See also Remark 5.1.

Brief heuristic. In cylindrical coordinates the Laplacian is written as

$$
\Delta_{x}=\partial_{r}^{2}+\partial_{z}^{2}+(N-2) \frac{\partial_{r}}{r} .
$$

Suppose that a solution to (1-1) is cylindrically symmetric and concentrated near the ring $(r, z) \sim\left(r_{0}, z_{0}\right)$. Then for an appropriately small $\lambda_{0}>0$ we may write

$$
u(t, x)=\frac{1}{\lambda_{0}} v\left(\frac{t}{\lambda_{0}^{2}}, \frac{(r, z)-\left(r_{0}, z_{0}\right)}{\lambda_{0}}\right),
$$

where the function $v$ is supported on the half-plane $(r, z) \in\left[-r_{0} / \lambda_{0}, \infty\right) \times \mathbb{R}$. Neglect that our parameters may vary in time. After changing coordinates, $v$ satisfies

$$
i \partial_{s} v+\Delta_{y} v+(N-2) \frac{\lambda_{0}}{r} \partial_{y_{1}} v+v|v|^{2}=0, \quad \text { where } s=\frac{t}{\lambda_{0}^{2}}, y=\frac{(r, z)-\left(r_{0}, z_{0}\right)}{\lambda_{0}} .
$$

For a solution $u(t, x)$ tightly concentrated near $\left(r_{0}, z_{0}\right)$, we might choose $\lambda_{0} \ll 1$ as the width of the window of concentration. Then, $(N-2)\left(\lambda_{0} / r\right) \partial_{y_{1}} v$ can be taken as a lower-order correction, and the evolution of $v$ is essentially that of the two-dimensional cubic NLS. If $v(s, y)$ falls within the robust $\log$-log blowup dynamic, we would expect the concentration near $\left(r_{0}, z_{0}\right)$ to increase, and for the lowerorder correction in (1-16) to become less relevant. 
We can identify our main challenge: to ensure persistence of sufficient decay in the original variables near $r=0$ such that conditions there mimic those at infinity during a log-log blowup of two-dimensional cubic NLS.

\section{Setting of the bootstrap}

In this section we identify data concentrated near the set $(r, z) \sim(1,0)$, according to properties we will later show persist. Our subsequent arguments are based on the two-dimensional $L^{2}$-critical log-log blowup dynamic, which has been comprehensively investigated in [Merle and Raphaël 2003; 2004; 2005a; 2005b; 2006; Raphaël 2005]. This work stems from those detailed studies.

Definition 2.1 (fundamental properties of almost self-similar profiles). For all $b>0$ sufficiently small, there exists a solution $\widetilde{Q}_{b} \in H^{1}\left(\mathbb{R}^{2}\right)$ of

$$
\Delta \widetilde{Q}_{b}-\widetilde{Q}_{b}+i b \Lambda \widetilde{Q}_{b}+\widetilde{Q}_{b}\left|\widetilde{Q}_{b}\right|^{2}=-\Psi_{b}
$$

that is supported on the ball of radius $2 /|b|$ and converges to $Q$ in $C^{3}\left(\mathbb{R}^{2}\right)$ as $b \rightarrow 0$. The profiles $\widetilde{Q}_{b}$ have mass of the order of $b^{2}$ larger than $Q$, and energy of the order $e^{-C / b}$. The truncation error $\Psi_{b}$ acts as the source of the linear radiation,

$$
\Delta \zeta_{b}-\zeta_{b}+i b \Lambda \zeta_{b}=\Psi_{b}
$$

The radiation $\zeta_{b}$ is not in $L^{2}$, with the precise decay rate $\Gamma_{b}=\lim _{|y| \rightarrow+\infty}|y|\left|\zeta_{b}\right|^{2}$. It is known that $\Gamma_{b} \sim e^{-\pi / b}$, and it is this decay property linked to the central profile $\widetilde{Q}_{b}$ that is responsible for the log-log rate of the two-dimensional $L^{2}$-critical problem. For our analysis, we will truncate $\zeta_{b}$ near $|y| \sim e^{+a / b}$ for a small fixed parameter $a$. See page 694 for details.

Lemma 2.2 (smoothness of $\widetilde{Q}_{b}$ ). The almost self-similar profiles $\widetilde{Q}_{b}$ are smooth. For any $s \geq 3$,

$$
\limsup _{b \rightarrow 0}\left\|\widetilde{Q}_{b}\right\|_{C^{s}\left(\mathbb{R}^{2}\right)}<+\infty \quad \text { and } \quad \limsup _{b \rightarrow 0}\left\|\widetilde{Q}_{b}\right\|_{H^{s}\left(\mathbb{R}^{2}\right)}<+\infty .
$$

Geometric decomposition. In place of $(r, z, \theta) \in \mathbb{R}^{N}$ we change coordinates to the rescaled half-plane:

$$
y=\frac{(r, z)-\left(r_{0}, z_{0}\right)}{\lambda_{0}} \in\left[-r_{0} / \lambda_{0},+\infty\right) \times \mathbb{R} .
$$

The fixed parameters $r_{0}, z_{0}, \lambda_{0}$ will later be replaced by $r(t), z(t)$, and $\lambda(t)$. This will be clear from the context. Note the measure due to cylindrical symmetry, $d x=\lambda_{0} \mu_{\lambda_{0}, r_{0}}(y) d y$ is given by

$$
\mu_{\lambda_{0}, r_{0}}(y)=\left|S^{N-2}\right|\left(\lambda_{0} y_{1}+r_{0}\right)^{N-2} \mathbb{1}_{y_{1} \geq-r_{0} / \lambda_{0}} .
$$

We will shortly hypothesize parameters of the decomposition in such a way that the support of both $\widetilde{Q}_{b}$ and $\tilde{\zeta}_{b}$ are well away from the boundary of domain (2-18). For convenience we will omit the constant factor $\left|S^{N-2}\right|$ and approximate $\mu(y) \sim 1$ on this region; see (2-55). Integrals in $y$ can then be seen as taken over all of $\mathbb{R}^{2}$, and regular integration by parts applies. Any integral that cannot be localized in this way will be treated separately, and very carefully.

To begin, we modulate suitable cylindrically symmetric data as if it were two-dimensional: 
Lemma 2.3 (existence of geometric decomposition at a fixed time [Raphaël 2006, Lemma 2]). Suppose that $v \in H_{\text {cyl }}^{1}\left(\mathbb{R}^{N}\right)$ may be written in the form

$$
v(r, z, \theta)=\frac{1}{\lambda_{v}}\left(\widetilde{Q}_{b_{v}}+\epsilon_{v}\right)\left(\frac{(r, z)-\left(r_{v}, z_{v}\right)}{\lambda}\right) e^{-i \gamma_{v}}
$$

for some parameters $\lambda_{v}, b_{v}, r_{v}>0$ and $\gamma_{v}, z_{v} \in \mathbb{R}$ such that

$$
\begin{gathered}
\int\left|\nabla_{y} \epsilon_{v}\right|^{2} \mu_{\lambda_{v}, r_{v}}(y) d y+\int_{|y| \leq 10 / b_{v}}\left|\epsilon_{v}\right|^{2} e^{-|y|} d y<\Gamma_{b_{v}}^{\frac{1}{2}}, \\
\left|\left(r_{v}, z_{v}\right)-(1,0)\right|<\frac{1}{3} \text { and } 10 \lambda_{v}<b_{v}<\alpha^{*} .
\end{gathered}
$$

Then there are nearby parameters $\lambda_{0}, b_{0}, r_{0}>0$ and $\gamma_{0}, z_{0} \in \mathbb{R}$ with

$$
\left|b_{0}-b_{v}\right|+\left|\frac{\lambda_{0}}{\lambda_{v}}-1\right|+\frac{\left|\left(r_{0}, z_{0}\right)-\left(r_{v}, z_{v}\right)\right|}{\lambda_{v}} \leq \Gamma_{b_{0}}^{\frac{1}{5}},
$$

such that the corresponding $\epsilon_{0}$,

$$
\epsilon_{0}(y)=\lambda_{0} v\left(\lambda_{0} y+\left(r_{0}, z_{0}\right)\right) e^{i \gamma_{0}}-\widetilde{Q}_{b_{0}},
$$

satisfies the two-dimensional orthogonality conditions ${ }^{4}$

$$
\operatorname{Re}\left(\epsilon_{0},|y|^{2} \widetilde{Q}_{b_{0}}\right)=\operatorname{Re}\left(\epsilon_{0}, y \widetilde{Q}_{b_{0}}\right)=\operatorname{Im}\left(\epsilon_{0}, \Lambda^{2} \widetilde{Q}_{b_{0}}\right)=\operatorname{Im}\left(\epsilon_{0}, \Lambda \widetilde{Q}_{b_{0}}\right)=0 .
$$

We now identify a neighborhood of the singular set, the complement of which is contiguous and includes both the origin and infinity. In the case of $N=3$, the singular set is a ring and the neighborhood a toroid. Define two smooth cutoff functions,

$$
\chi(r, z, \theta)=\left\{\begin{array}{ll}
1 & \text { for }|(r, z)-(1,0)| \geq \frac{2}{3}, \\
0 & \text { for }|(r, z)-(1,0)| \leq \frac{1}{3},
\end{array} \quad \chi_{0}(r, z, \theta)= \begin{cases}1 & \text { for }|(r, z)-(1,0)| \geq \frac{1}{7}, \\
0 & \text { for }|(r, z)-(1,0)| \leq \frac{1}{8} .\end{cases}\right.
$$

In Section 4 we will define a further series of cutoff functions $\psi$ and $\varphi$, supported on bounded regions where $\chi_{0} \equiv 1$. We now describe the initial data for our bootstrap procedure.

Definition 2.4 (description of initial data $\mathscr{P}$ ). For $\alpha^{*}>0$ a constant to be determined, let $\mathscr{P}\left(\alpha^{*}\right)$ be the set of cylindrically symmetric $u_{0} \in H_{\text {cyl }}^{N}\left(\mathbb{R}^{N}\right)$ that can be written in the form

$$
\begin{aligned}
u_{0}(r, z) & =\frac{1}{\lambda_{0}}\left(\widetilde{Q}_{b_{0}}+\epsilon_{0}\right)\left(\frac{(r, z)-\left(r_{0}, z_{0}\right)}{\lambda_{0}}\right) e^{-i \gamma_{0}} \\
& =\frac{1}{\lambda_{0}}\left(\widetilde{Q}_{b_{0}}\right)\left(\frac{(r, z)-\left(r_{0}, z_{0}\right)}{\lambda_{0}}\right) e^{-i \gamma_{0}}+\tilde{u}_{0}(r, z),
\end{aligned}
$$

in a way that satisfies the following two sets of conditions:

\footnotetext{
${ }^{4}$ The decomposition of [Merle and Raphaël 2003] used slightly different orthogonality conditions. Equation (2-25) is the decomposition introduced [Merle and Raphaël 2004, Lemma 6], which leads to a better estimate on the phase parameter than was achieved in the former paper.
} 


\section{Singularity of a log-log nature:}

C1.1. Radial profile is focused near a singular ring:

$$
\left|\left(r_{0}, z_{0}\right)-(1,0)\right|<\alpha^{*} .
$$

C1.2. Radial profile is close to $Q$ near the singular ring: The profiles $\widetilde{Q}_{b}$ have nearly the mass of $Q$ and account for nearly all mass globally:

$$
0<b_{0}+\left\|\tilde{u}_{0}\right\|_{L^{2}\left(\mathbb{R}^{N}\right)}<\alpha^{*} ;
$$

$\epsilon_{0}(y)$ satisfies both the orthogonality conditions

$$
\operatorname{Re}\left(\epsilon_{0},|y|^{2} \widetilde{Q}_{b_{0}}\right)=\operatorname{Re}\left(\epsilon_{0}, y \widetilde{Q}_{b_{0}}\right)=\operatorname{Im}\left(\epsilon_{0}, \Lambda^{2} \widetilde{Q}_{b_{0}}\right)=\operatorname{Im}\left(\epsilon_{0}, \Lambda \widetilde{Q}_{b_{0}}\right)=0
$$

and the smallness condition

$$
\int\left|\nabla_{y} \epsilon_{0}(y)\right|^{2} \mu_{\lambda_{0}, r_{0}}(y) d y+\int_{|y| \leq \frac{10}{b_{0}}}\left|\epsilon_{0}(y)\right|^{2} e^{-|y|} d y<\Gamma_{b_{0}}^{\frac{6}{7}} .
$$

C1.3. Conformal and scaling parameters are consistent with log-log blowup speed:

$$
e^{-e^{\frac{2 \pi}{b_{0}}}}<\lambda_{0}<e^{-e^{\frac{\pi}{2} \frac{1}{b_{0}}}}
$$

C1.4. Energy and localized momentum are normalized:

$$
\lambda_{0}^{2}\left|E_{0}\right|+\lambda_{0}\left|\operatorname{Im}\left(\int \nabla_{x} \psi^{(x)} \cdot \nabla_{x} u_{0} \bar{u}_{0}\right)\right|<\Gamma_{b_{0}}^{10},
$$

where $\psi^{(x)}$ is a cylindrically symmetric smooth cutoff function given by

$$
\psi^{(x)}(r, z, \theta)= \begin{cases}r+z & \text { for }|(r, z)-(1,0)| \leq \frac{1}{2}, \\ 0 & \text { for }|(r, z)-(1,0)| \geq \frac{3}{4} .\end{cases}
$$

\section{Regularity away from the singularity:}

C2.1. Scaling-consistent $\dot{H}^{N}$ norm:

$$
\left\|u_{0}\right\|_{H^{N}\left(\mathbb{R}^{N}\right)}<\frac{C_{\widetilde{Q}}}{\lambda_{0}^{N}},
$$

where $C_{\widetilde{Q}}$ is a universal constant due to Lemma 2.2.

C2.2. Strong hierarchy of regularity away from the singular ring:

$$
\left\|\chi_{0} u_{0}\right\|_{H^{N-\kappa}\left(\mathbb{R}^{N}\right)}<\frac{1}{\lambda_{0}^{N-2 \kappa}},
$$

for each half-integer $\frac{1}{2} \leq \kappa \leq \frac{N}{2}$.

C2.3. Vanishing lower-order norms away from the singular ring:

$$
\left\|\chi_{0} u_{0}\right\|_{H^{\frac{N}{2}-\frac{1}{2}}} 1_{\left(\mathbb{R}^{N}\right)}<\left(\alpha^{*}\right)^{\frac{1}{2}} .
$$


Lemma 2.3 guarantees that $\mathscr{P}\left(\alpha^{*}\right)$ is open in $H_{\text {cyl }}^{1} \cap H_{\text {cyl }}^{N}$. See the Appendix for a proof that $\mathscr{P}\left(\alpha^{*}\right)$ is nonempty.

For the remainder of this paper, fix an arbitrary $u_{0} \in \mathscr{P}\left(\alpha^{*}\right)$. Let $u(t)$ denote the evolution under (1-1), with maximum (forward) lifetime $T_{\max }>0$, possibly infinite.

Continuous evolution in $H^{N}\left(\mathbb{R}^{N}\right)$ implies the same in $H^{1}\left(\mathbb{R}^{N}\right)$, so by Lemma 2.3 there is some $T_{\text {geo }} \in$ $\left(0, T_{\max }\right]$ - which may be assumed maximal — for which the geometric decomposition of Lemma 2.3 can be applied on $\left[0, T_{\mathrm{geo}}\right)$. There exist unique continuous functions $\lambda(t), b(t), r(t):\left[0, T_{\mathrm{geo}}\right) \rightarrow(0, \infty)$ and $\gamma(t), z(t):\left[0, T_{\text {geo }}\right) \rightarrow \mathbb{R}$, with the expected initial values, where

$$
\begin{aligned}
u(t, r, z, \theta) & =\frac{1}{\lambda(t)}\left(\widetilde{Q}_{b(t)}+\epsilon(t)\right)\left(\frac{(r, z)-(r(t), z(t))}{\lambda}\right) e^{-i \gamma(t)} \\
& =\frac{1}{\lambda(t)}\left(\widetilde{Q}_{b(t)}\right)\left(\frac{(r, z)-(r(t), z(t))}{\lambda}\right) e^{-i \gamma(t)}+\tilde{u}(t, r, z, \theta),
\end{aligned}
$$

such that $\epsilon(t, y)$ satisfies the two-dimensional orthogonality conditions

$$
\begin{aligned}
\operatorname{Re}\left(\epsilon(t),|y|^{2} \widetilde{Q}_{b(t)}\right) & =0, \\
\operatorname{Re}\left(\epsilon(t), y \widetilde{Q}_{b(t)}\right) & =0, \\
\operatorname{Im}\left(\epsilon(t), \Lambda^{2} \widetilde{Q}_{b(t)}\right) & =0, \\
\operatorname{Im}\left(\epsilon(t), \Lambda \widetilde{Q}_{b(t)}\right) & =0 .
\end{aligned}
$$

We may now define the rescaled time,

$$
s(t)=\int_{0}^{t} \frac{1}{\lambda^{2}(\tau)} d \tau+s_{0}, \quad \text { where } s_{0}=e^{\frac{3 \pi}{4 b_{0}}} .
$$

The choice of $s_{0}$ will prove convenient in the proof of Lemma 3.15 (page 699).

Also set $s_{1}=s\left(T_{\text {hyp }}\right)$, for $T_{\text {hyp }}$ as in Definition 2.6 below.

Notation 2.5 (fixed parameters). To aid the reader, we provide a brief summary of the various parameters that will be introduced, in the order one might ultimately determine them:

- $\eta$ and $a$ are parameters that determine the cutoff shape of $\widetilde{Q}_{b}$ and $\tilde{\zeta}_{b}$; see (3-80) and (3-104). The value of $a>0$ is assumed sufficiently small for the proof of Lemma 3.22, relative to some universal constant. Before that, the proof of Lemma 3.19 is conditioned on the choice of $\eta<a / C_{0}$, for another universal constant $C_{0}>0$; see (3-196). These choices affect the class of initial data $\mathscr{P}$, both by setting the profiles $\widetilde{Q}_{b}$ and by forcing an upper bound on the value of $\alpha^{*}$.

- $\sigma_{1}, \sigma_{2}$ and $\sigma_{3}$ : parameters in the statements of Lemma 4.1, Lemma 4.3, and Corollary 4.4. Their value is chosen (repeatedly) according to circumstance.

- $\sigma_{4}$ : an arbitrary universal constant, $0<\sigma_{4} \ll 1$, used in the proof of Lemma 4.8.

- $\sigma_{5}$ : defined for Lemma 4.8. Its value depends on $\sigma_{4}$, and is uniform over all $m>0$ small enough.

- $m^{\prime}$ : existence of $m^{\prime}<m$ with particular properties in a key assertion of Proposition 2.8. Some particular value $m^{\prime} \in\left(m-\sigma_{5} / 2, m\right)$ is chosen for the proof of Lemma 4.10. 
- $\sigma_{6}$ : parameter in the statement of Lemma 4.11. Its value is fixed for the proof of Lemma 4.13.

- $\sigma_{7}$ : defined for Lemma 4.17. Its value is uniform over all $m>0$ small enough.

- $m$ : a fixed constant $m>0$ that features in the bootstrap hypotheses of Definition 2.6. For the purpose of various proofs in Section 4, $m$ will be assumed sufficiently small. The exact value of $m$ may be determined apriori, and will affect the class of initial data $\mathscr{P}$ by forcing an upper bound on the value of $\alpha^{*}$.

- $\alpha^{*}$ : A fixed positive constant to be determined last. For the purpose of various proofs throughout this paper, $\alpha^{*}$ will be assumed sufficiently small.

The following bootstrap hypotheses are possible due to our choice of data in $\mathscr{P}$.

Definition 2.6 (time $T_{\text {hyp }}>0$ and bootstrap hypotheses). Let $0<T_{\text {hyp }} \leq T_{\max }$ be the maximum time such that for all $t \in\left[0, T_{\text {hyp }}\right)$ the following two sets of conditions hold:

\section{Singularity remains of a log-log nature:}

H1.1. Profile remains focused near a singular ring:

$$
|(r(t), z(t))-(1,0)|<\left(\alpha^{*}\right)^{\frac{1}{2}}
$$

H1.2. Profile remains close to $Q$ near the singular ring:

$$
\begin{gathered}
0<b(t)+\|\tilde{u}(t)\|_{L^{2}\left(\mathbb{R}^{N}\right)}<\left(\alpha^{*}\right)^{\frac{1}{10}}, \\
\int\left|\nabla_{y} \epsilon(t)\right|^{2} \mu_{\lambda(t), r(t)}(y) d y+\int_{|y| \leq \frac{10}{b(t)}}|\epsilon(t)|^{2} e^{-|y|} d y \leq \Gamma_{b(t)}^{\frac{3}{4}} .
\end{gathered}
$$

H1.3. Conformal and scaling parameters remain consistent with log-log blowup speed:

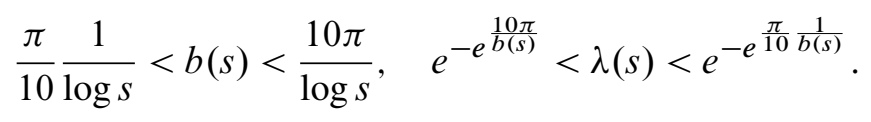

H1.4. Energy and localized momentum remain normalized:

$$
\lambda^{2}(t)\left|E_{0}\right|+\lambda(t)\left|\operatorname{Im}\left(\int \nabla \psi^{(x)} \cdot \nabla u(t) \bar{u}(t)\right)\right|<\Gamma_{b(t)}^{2} .
$$

H1.5. Norm growths are almost monotonic:

$$
\lambda\left(s_{b}\right) \leq 3 \lambda\left(s_{a}\right) \text { for all } s_{a} \leq s_{b} \in\left[s_{0}, s_{1}\right] .
$$

\section{Regularity away from the singularity persists:}

H2.1. Growth of $\dot{H}^{N}$ is near scaling:

$$
\|u(t)\|_{H^{N}\left(\mathbb{R}^{N}\right)}<\frac{e^{+\frac{m}{b(t)}}}{\lambda^{N}(t)}
$$


H2.2. Strong hierarchy of regularity away from the singular ring persists:

$$
\|\chi u(t)\|_{H^{N-\kappa}}<\frac{e^{+(1+\kappa) \frac{m}{b(t)}}}{\lambda^{N-2 \kappa}(t)}
$$

for each half-integer $\frac{1}{2} \leq \kappa<\frac{N}{2}$, and

$$
\|\chi u(t)\|_{H^{\frac{N}{2}}}<e^{+\frac{2 m+\pi}{b(t)}} .
$$

H2.3. Lower-order norms away from the singular ring remain bounded:

$$
\|\chi u(t)\|_{H^{\frac{N}{2}-\frac{1}{2}}}<\left(\alpha^{*}\right)^{\frac{1}{10}} .
$$

An important consequence of H1.2, H1.3, and the forthcoming estimate on $\Gamma_{b}$, (3-103), is that

$$
\lambda(t)<e^{-e^{\frac{\pi}{10 b(t)}}}<\Gamma_{b(t)}^{10} .
$$

Therefore as a consequence of H1.1 and the definition of $A$ in (3-104) below,

$$
\frac{2}{3} \leq \mu(y) \leq \frac{3}{2} \text { for all }|y| \leq 5 A(t) .
$$

The region $|y| \leq 5 A(t)$ is exceptionally wide, encompassing the support of both the central profile $\widetilde{Q}_{b}$ and the associated radiation $\tilde{\zeta}_{b}$.

Remark 2.7 (geometric decomposition is well defined). Hypotheses H1.1-H1.5 easily satisfy the conditions of Lemma 2.3, ensuring that $T_{\text {hyp }} \leq T_{\text {geo }}$ and the unique geometric decomposition (2-38) is available.

Proposition 2.8 (bootstrap conclusion). For $\alpha^{*}>0$ sufficiently small, hypotheses (2-44)-(2-53) are not sharp. There exists $m^{\prime}<m$ such that, for all $t \in\left[0, T_{\text {hyp }}\right)$ :

I1.1.

$$
\begin{gathered}
|(r(t), z(t))-(1,0)|<\left(\alpha^{*}\right)^{\frac{2}{3}} . \\
0<b(t)+\|\tilde{u}(t)\|_{L^{2}\left(\mathbb{R}^{N}\right)}<\left(\alpha^{*}\right)^{\frac{1}{5}},
\end{gathered}
$$$$
\int\left|\nabla_{y} \epsilon(t)\right|^{2} \mu_{\lambda(t), r(t)}(y) d y+\int_{|y| \leq \frac{10}{b(t)}}|\epsilon(t)|^{2} e^{-|y|} d y \leq \Gamma_{b(t)}^{\frac{4}{5}} .
$$$$
\frac{\pi}{5} \frac{1}{\log S}<b(s)<\frac{5 \pi}{\log s}, \quad e^{-e^{\frac{5 \pi}{b(t)}}}<\lambda(t)<e^{-e^{\frac{\pi}{5} \frac{1}{b(t)}}} .
$$

$$
\lambda^{2}(t)\left|E_{0}\right|+\lambda(t)\left|\operatorname{Im}\left(\int \nabla \psi^{(x)} \cdot \nabla u(t) \bar{u}(t)\right)\right|<\Gamma_{b(t)}^{4} .
$$

I1.5.

$$
\lambda\left(s_{b}\right) \leq 2 \lambda\left(s_{a}\right) \quad \text { for all } s_{a} \leq s_{b} \in\left[s_{0}, s_{1}\right] .
$$

I2.1.

$$
\|u(t)\|_{H^{N}\left(\mathbb{R}^{N}\right)}<\frac{e^{+\frac{m^{\prime}}{b(t)}}}{\lambda^{N}(t)} .
$$


I2. 2 .

$$
\|\chi u(t)\|_{H^{N-\kappa}}<\frac{e^{+(1+\kappa) \frac{m^{\prime}}{b(t)}}}{\lambda^{N-2 \kappa}(t)}
$$

for each half-integer $\frac{1}{2} \leq \kappa<\frac{N}{2}$, and

I2.3.

$$
\begin{gathered}
\|\chi u(t)\|_{H^{\frac{N}{2}}}<e^{+\frac{2 m^{\prime}+\pi}{b(t)}} . \\
\|\chi u(t)\|_{H^{\frac{N}{2}-\frac{1}{2}}}<\left(\alpha^{*}\right)^{\frac{1}{5}} .
\end{gathered}
$$

As a consequence, $T_{\text {hyp }}=T_{\text {max }}$.

Strategy of proof: the log-log argument. We will establish statements I1.1-I1.5 in Section 3 using the arguments of [Merle and Raphaël 2003; 2006]. Here we identify the main challenge in maintaining the log-log dynamics. As with all modulation arguments, we seek to reduce the question of blowup to a finite-dimensional ODE dynamic for the parameters. This is only possible due to the algebraic structure associated with $Q$. Recall the operator $\Lambda=1+y \cdot \nabla_{y}$, which one might recognize from either the argument $E(Q)=0$ :

$$
(0, \Lambda(Q))=\left(\Delta Q-Q+Q|Q|^{2}, \Lambda(Q)\right)=-2 E(Q),
$$

or from the Pohozaev identity:

$$
(0, \Lambda(v))=\operatorname{Re}\left(i v_{s}+\Delta_{y} v+v|v|^{2}, \Lambda(v)\right)=-\frac{1}{2} \frac{d}{d s} \operatorname{Im} \int v y \cdot \nabla \bar{v} d y-2 E(v),
$$

which is also a consequence of formally calculating the virial identity's term

$$
\frac{d^{2}}{d^{2} s} \int|y|^{2}|v|^{2} d y
$$

Substitution of (2-38) into (1-1) will produce an equation for $\epsilon$. Ignoring the distinction between $Q$ and $\widetilde{Q}_{b}$, the terms linear in $\epsilon$ are $i \partial_{s} \epsilon+L(\epsilon)$, where $L$ is the linearized propagator near $Q$. As a matrix on real and imaginary parts,

$$
L(\epsilon)=\left[\begin{array}{cc}
0 & L_{-} \\
-L_{+} & 0
\end{array}\right]\left[\begin{array}{c}
\epsilon_{\mathrm{re}} \\
i \epsilon_{\mathrm{im}}
\end{array}\right] \text { with }\left\{\begin{array}{l}
L_{+}=-\Delta+1-3 Q^{2} \\
L_{-}=-\Delta+1-Q^{2}
\end{array}\right.
$$

Weinstein [1985] noted that

$$
L_{-}\left(|y|^{2} Q\right)=-2 \Lambda Q, \quad L_{-}(y Q)=-2 \nabla Q, \quad \text { and } \quad L_{+}(\Lambda Q)=-2 Q
$$

These algebraic properties are the inspiration for the orthogonality conditions, so that, by taking appropriate inner products of the $\epsilon$-equation, linear terms cancel. For example, the imaginary part of the inner product with $|y|^{2} Q$ has no linear terms due to conditions (2-39) and (2-42). The imaginary part of the inner product with $y Q$ is controlled by the momentum.

The most fruitful calculation is when we take the real part of an inner product of the $\epsilon$-equation with $\Lambda Q$. This is of course a localized version of (2-67). We substitute conservation of energy to eliminate 
the linear term, $2 \operatorname{Re}(\epsilon, Q)$, which is due to the third identity of (2-69). The remaining terms quadratic in $\epsilon$ form the following,

$$
H(\epsilon, \epsilon)=\left[\begin{array}{cc}
\mathscr{L}_{\mathrm{re}} & 0 \\
0 & \mathscr{L}_{\mathrm{im}}
\end{array}\right]\left[\begin{array}{c}
\epsilon_{\mathrm{re}} \\
i \epsilon_{\mathrm{im}}
\end{array}\right] \cdot\left[\begin{array}{c}
\epsilon_{\mathrm{re}} \\
-i \epsilon_{\mathrm{im}}
\end{array}\right] \text { with }\left\{\begin{array}{l}
\mathscr{L}_{\mathrm{re}}=-\Delta+3 Q y \cdot \nabla Q \\
\mathscr{L}_{\mathrm{im}}=-\Delta+Q y \cdot \nabla Q .
\end{array}\right.
$$

The operator $H(\epsilon, \epsilon)$ is the derivative with respect to scaling of the conserved energy of the linear flow. It has coercivity properties that mirror the stability of $Q$ :

Proposition 2.9 (spectral property). There exists a universal constant $\delta_{0}>0$ such that, for any $v \in H^{1}$,

$$
\begin{aligned}
& H(v, v) \geq \delta_{0}\left(\int_{y \in \mathbb{R}^{2}}\left|\nabla_{y} v\right|^{2}+\int_{y \in \mathbb{R}^{2}}\left|v^{2}\right| e^{-|y|}\right)-\frac{1}{\delta_{0}} \times \\
& \left((\operatorname{Re}(v, Q))^{2}+(\operatorname{Re}(v, \Lambda Q))^{2}+(\operatorname{Re}(v, y Q))^{2}+(\operatorname{Im}(v, \Lambda Q))^{2}+\left(\operatorname{Im}\left(v, \Lambda^{2} Q\right)\right)^{2}+(\operatorname{Im}(v, \nabla Q))^{2}\right) .
\end{aligned}
$$

The two-dimensional spectral property as stated here has a numerical proof [Fibich et al. 2006]. ${ }^{5}$ Assuming we can ensure $H$ is coercive, the goal is to prove the local virial identity

$$
b_{s} \geq \delta_{1}\|\epsilon\|-\Gamma_{b}^{1-C \eta},
$$

where we have defined

$$
\|\epsilon\|=\int\left|\nabla_{y} \epsilon\right|^{2} \mu d y+\int_{|y| \leq \frac{10}{b}}|\epsilon|^{2} e^{-|y|} d y .
$$

To prove (2-72) using the spectral property requires that we control the contribution from all other terms of the conservation of energy. In particular, we must establish nonlocal control:

$$
\int_{\mathbb{R}^{2}}|\epsilon(y)|^{4} \mu(y) \ll \int_{\mathbb{R}^{2}}\left|\nabla_{y} \epsilon\right|^{2} \mu(y) .
$$

This is our main challenge.

The local virial identity (2-72) is a satisfactory control for $\epsilon$ at times where $b_{s}<0$. However, our argument is based on approximating the central profile of the solution; therefore we cannot expect monotinicity in our modulation parameters. Including the radiation $\tilde{\zeta}$ to better approximate the central profile, repeating the local virial calculation, and taking into account the mass flux leaving the support of the radiation, Merle and Raphaël [2006] discovered a Lyapunov functional. It is remarkable that we can approximate the Lyapunov functional very precisely in terms of a positive multiple of a norm of $\epsilon$. The functional is then used to bridge the control of $\epsilon$ between times where $b_{s}<0$. The approximation here is achieved through the conservation of energy, and involves (2-74) a second time.

Regarding (2-74), change variables to get

$$
\int_{\mathbb{R}^{2}}|\epsilon(y)|^{4} \mu(y)=\lambda^{2} \int_{\mathbb{R}^{N}}|\tilde{u}|^{4}=\lambda^{2} \int_{\mathbb{R}^{N}}|\chi \tilde{u}|^{4}+\lambda^{2} \int_{\mathbb{R}^{N}}\left(1-\chi^{4}\right)|\tilde{u}|^{4} .
$$

${ }^{5}$ The numerical proof is given for the $L^{2}$-critical nonlinearities of Theorem 1.1 in dimensions $d=2,3,4$, and in $d=5$ with a slight change of orthogonality conditions. In dimension $d=1$ the proof is explicit [Merle and Raphaël 2005a, Proposition 2]. 
Since the support of $\chi$ includes the origin, we must apply $N$-dimensional Sobolev to that term:

$$
\|\chi \tilde{u}\|_{L^{4}\left(\mathbb{R}^{N}\right)}^{4} \lesssim\|\chi u\|_{H^{\frac{N}{2}-1}\left(\mathbb{R}^{N}\right)}^{2}\|\chi u\|_{H^{1}\left(\mathbb{R}^{N}\right)}^{2} .
$$

Change variables again and observe that to achieve (2-74) requires at least that $\|\chi u\|_{H^{\frac{N}{2}-1}\left(\mathbb{R}^{N}\right)} \ll 1$.

Strategy of proof: persistence of regularity. Once we have established the log-log nature of our blowup, we expect powers of $1 / \lambda$ to be as integrable in time as powers of

$$
\sqrt{\frac{\log \left|\log \left(T_{\max }-t\right)\right|}{T_{\max }-t}} .
$$

Indeed, as noted in [Raphaël and Szeftel 2009],

$$
\int_{0}^{t} \frac{d \tau}{\lambda^{\mu}(\tau)} \leq C(\delta) \frac{1}{\lambda^{\mu-2+\delta}(t)}
$$

for any $\delta>0$ and $v>2$. Our argument cannot allow any loss of scaling. At all times we must be cautious to account for all factors of $e^{\frac{1}{b}}$. We prove that

$$
\int_{0}^{t} \frac{e^{\frac{\sigma^{*}}{b(\tau)}}}{\lambda^{\mu}(\tau)} d \tau \leq C\left(\sigma^{*}, \sigma, v\right) \frac{e^{\frac{\sigma^{*}+\sigma}{b(t)}}}{\lambda^{\mu-2}(t)},
$$

for any $\sigma^{*}<\sigma^{*}+\sigma$ of either sign and $\mu>2$. The arguments of Section 4 , to establish statements I2.1-I2.3, proceed in three stages.

Control of $\|u\|_{H^{N}}$. We explicitly calculate $\frac{d}{d t}\left\|\nabla^{N} u\right\|_{L^{2}}^{2}$ and seek to estimate the resulting error terms separately in two regions of space. First, away from the singularity, on the truly $N$-dimensional region that includes the origin, the estimates are simpler, due to hypotheses H2.2 and H2.3. Second, on a neighborhood of the singular set, things are more delicate, and we split the solution into the rescaled almost self-similar profile and $\tilde{u}$, defined in (2-38). Since $\widetilde{Q}_{b}$ is smooth, the higher-order norms scale exactly with $1 / \lambda$. In particular,

$$
\left\|\frac{1}{\lambda} \widetilde{Q}_{b}(y)\right\|_{H^{N}\left(\mathbb{R}^{N}\right)} \leq \frac{C\left(\widetilde{Q}_{b}\right)}{\lambda^{N}(t)},
$$

where the constant is uniform for all $b$ sufficiently small; see Lemma 2.2. Note that (2-77) is better than H2.1. For terms in $\tilde{u}$, the $H^{1}$ norm is better than $1 / \lambda$, due to H1.3. By assuming $m>0$ is small enough, we use this superior $H^{1}$ control to offset the logarithmic loss due to our use of H2.1. We prove that

$$
\left|\frac{d}{d t}\|u\|_{H^{N}}^{2}\right| \lesssim \frac{1}{\lambda^{2 N+2}}+\frac{e^{-\frac{\sigma_{5}}{b}}}{\lambda^{2}}\|u\|_{H^{N}}^{2} .
$$

To prove I2.1, we integrate carefully with (2-76).

Remark 2.10. The exact scaling of the smooth central profile was not needed by Raphaël and Szeftel [2009] to control a higher order norm. With the Strauss radial embedding, those authors prove an estimate analogous to $\frac{d}{d t}\|u\|_{H^{N}}^{2} \lesssim\|u\|_{H^{N}}^{2-\delta}\|u\|_{H^{1}}^{N \delta+2}$, which is compatible with (2-75) and a hypothesis of the form $\|u\|_{H^{N}} \lesssim 1 / \lambda^{N+C(\delta)}$. 
Initial regularity improvement. Let $\psi^{A}$ be a smooth cutoff function that covers the support of $\nabla \chi-$ this includes the boundary of a neighborhood of the singular set that acts as an interface between the singular dynamics and the truly $N$-dimensional dynamics. We hope for any control of $\left\|\psi^{A} u\right\|_{H^{v}}$ that is better than an interpolation of H2.1. Calculate $\frac{d}{d t}\left\|\psi^{A} u\right\|_{H^{v}}^{2}$ directly from (1-1) and integrate in time. The result is effectively Kato's smoothing effect and a Strichartz estimate,

$$
\left\|\psi^{A} u\right\|_{L_{t}^{\infty} H^{v}}^{2} \lesssim\left\|\psi^{B} u\right\|_{L_{t}^{2} H^{v+\frac{1}{2}}}^{2}+\int_{0}^{t}\left|\int D^{v}\left(\psi^{A} u|u|^{2}\right) D^{v}\left(\psi^{A} \bar{u}\right)\right|,
$$

where $\psi^{B}$ is some other cutoff function with slightly larger support.

Due to (2-76), we see that the term in $H^{v+\frac{1}{2}}$ is in fact of the order $1 / \lambda^{2\left(v-\frac{1}{2}\right)}$. This is exactly the sort of control we want, but the nonlinear term of (2-78) is uncooperative.

To estimate the nonlinear term of (2-78) we prove a modified Brezis-Gallouët estimate that does not break scaling too badly, the proof of which requires hypothesis H2.1 to be scaling consistent up to a sufficiently small power of $e^{\frac{1}{b}}$. See Remark 4.15. This delicacy is not required in the radial case [Raphaël 2006; Raphaël and Szeftel 2009] as Strauss's radial embedding is already scaling consistent. In place of a Brezis-Gallouët estimate, Holmer and Roudenko [2011] use an elegant microlocal estimate to smooth the nonlocal part of the nonlinearity.

Iterated smoothing. The next stage is to prove I2.2 and I2.3 hold on the support of $\nabla \chi$. We iterate the argument of (2-78), in half-integer steps, beginning with $v=N-\frac{1}{2}$, and introducing a new cutoff with smaller support each time. Due to the initial regularity improvement, it is possible to handle the nonlinear term of (2-78) systematically, and at the same order as the term in $H^{v+\frac{1}{2}}$. Due to integration (2-76), at each stage we may smooth (almost) a half-derivative farther, relative to scaling, than was proved in the previous stage. After $N$ iterates, we find that $\left\|\psi^{C} u\right\|_{H^{\frac{N}{2}}}$ is (almost) order-zero in $\frac{1}{\lambda}$. The final iterate proves $\left\|\psi^{D} u\right\|_{H^{\frac{N}{2}-\frac{1}{2}}}$ is constant.

To complete the proof of I2.2 and I2.3, we repeat the iteration scheme for $\chi u$. The combination of hypotheses $\mathrm{H} 2.2$ and $\mathrm{H} 2.3$ with the results of the first iteration make the second iteration substantially simpler.

\section{Proof of log-log singular behavior}

In this section we will prove that properties I1.1-I1.5 are a consequence of hypotheses H1.1-H1.5 and the bound

$$
\|\chi u(t)\|_{H^{\frac{N}{2}-1}}<\left(\alpha^{*}\right)^{\frac{1}{10}}
$$

which is a particular consequence of $\mathrm{H} 2.3$.

Almost self-similar profiles. The parameter $\eta>0$ about to be used is universal, sufficiently small, and will be determined later on (see after (3-183) and after (3-196)). For $b \neq 0$, let

$$
R_{b}=\frac{2}{b} \sqrt{1-\eta} \text { and } R_{b}^{-}=R_{b} \sqrt{1-\eta},
$$


and let $\phi_{b}$ denote a radially symmetric cutoff function with

$$
\phi_{b}(y)=\left\{\begin{array}{ll}
1 & \text { for }|y| \leq R_{b}^{-}, \\
0 & \text { for }|y| \geq R_{b},
\end{array} \quad \text { and } \quad\left|\nabla \phi_{b}\right|_{L^{\infty}}+\left|\Delta \phi_{b}\right|_{L^{\infty}} \rightarrow 0 \text { as }|b| \rightarrow 0 .\right.
$$

The following result was originally shown in [Merle and Raphaël 2003, Proposition 1]. The refined cutoff, with parameter $\eta$, is introduced in [Merle and Raphaël 2004, Propositions 8 and 9].

Proposition 3.1 (localized self-similar profiles). For all $\eta>0$ sufficiently small there exists positive $b^{*}(\eta)$ and $\delta(\eta)$ such that for all $|b|<b^{*}(\eta)$ there exists a unique radial solution $Q_{b}$ to,

$$
\left\{\begin{array}{l}
\Delta Q_{b}-Q_{b}+i b \Lambda Q_{b}+Q_{b}\left|Q_{b}\right|^{2}=0, \\
P_{b}=Q_{b} e^{i \frac{b|y|^{2}}{4}}>0 \text { for } y \in\left[0, R_{b}\right), \\
\left|Q_{b}(0)-Q(0)\right|<\delta(\eta), \quad Q_{b}\left(R_{b}\right)=0 .
\end{array}\right.
$$

The truncation to $|y|<\frac{2}{b}, \widetilde{Q}_{b}(y)=Q_{b}(y) \phi_{b}(y)$, satisfies

$$
\Delta \widetilde{Q}_{b}-\widetilde{Q}_{b}+i b \Lambda \widetilde{Q}_{b}+\widetilde{Q}_{b}\left|\widetilde{Q}_{b}\right|^{2}=-\Psi_{b}
$$

with the explicit error term

$$
-\Psi_{b}=Q_{b} \Delta \phi_{b}+2 \nabla \phi_{b} \cdot \nabla Q_{b}+i b Q_{b} y \cdot \nabla \phi_{b}+\left(\phi_{b}^{3}-\phi_{b}\right) Q_{b}\left|Q_{b}\right|^{2} .
$$

Moreover, $\widetilde{Q}_{b}$ satisfies the following properties:

- Uniform closeness to the ground state:

$$
\left\|e^{C|y|}\left(\widetilde{Q}_{b}-Q\right)\right\|_{C^{3}} \rightarrow 0 \quad \text { as } b \rightarrow 0 .
$$

- Derivative with respect to $b$ :

$$
\left\|e^{C|y|}\left(\frac{\partial}{\partial b} \widetilde{Q}_{b}+i \frac{|y|^{2}}{4} Q\right)\right\|_{C^{2}} \rightarrow 0 \quad \text { as } b \rightarrow 0 .
$$

- Supercritical mass:

$$
\left.\frac{d}{d\left(b^{2}\right)}\left(\int\left|\widetilde{Q}_{b}\right|^{2}\right)\right|_{b^{2}=0}=d_{0} \quad \text { with } 0<d_{0}<+\infty .
$$

As a consequence of (3-85), for any polynomial $P(y)$ and $k=0,1$,

$$
\left|P(y) \nabla^{k} \Psi_{b}\right|_{L^{\infty}} \leq e^{-\frac{C(P)}{|b|}} .
$$

In particular, energy and momentum are degenerate:

$$
\left|E\left(\widetilde{Q}_{b}\right)\right| \leq e^{-(1-C \eta) \frac{\pi}{|b|}} \quad \text { and } \quad \operatorname{Im}\left(\int \nabla_{y} \widetilde{Q}_{b} \overline{\widetilde{Q}_{b}}\right)=0 .
$$


The linearized Schrödinger operator near $\widetilde{Q}_{b}$ is, $M\left[\begin{array}{c}v \\ i w\end{array}\right]=M_{+}(v, w)+i M_{-}(v, w)$, with,

$$
\begin{aligned}
& M_{+}(v, w)=-\Delta_{y} v+v-\left(\frac{\widetilde{Q}_{b}^{2}}{\left|\widetilde{Q}_{b}\right|^{2}}+2\right)\left|\widetilde{Q}_{b}\right|^{2} v-\operatorname{Im}\left(\widetilde{Q}_{b}^{2}\right) w \\
& M_{-}(v, w)=-\Delta_{y} w+w-\left(2-\frac{\widetilde{Q}_{b}^{2}}{\left|\widetilde{Q}_{b}\right|^{2}}\right)\left|\widetilde{Q}_{b}\right|^{2} w-\operatorname{Im}\left(\widetilde{Q}_{b}^{2}\right) v .
\end{aligned}
$$

As with $L$ from (2-68), there is an associated bilinear operator

$$
H_{b}(\epsilon, \epsilon)=H(\epsilon, \epsilon)+\tilde{H}_{b}(\epsilon, \epsilon)
$$

where $H(\epsilon, \epsilon)$ is the usual form (2-70) associated with $L$. The correction term may be written as

$$
\tilde{H}_{b}(\epsilon, \epsilon)=\int V_{11} \epsilon_{\mathrm{re}}{ }^{2}+\int V_{12} \epsilon_{\mathrm{re}} \epsilon_{\mathrm{im}}+\int V_{22} \epsilon_{\mathrm{im}}{ }^{2},
$$

for well-localized potentials built on $\widetilde{Q}_{b}, Q$ and $y \cdot \nabla$; see [Merle and Raphaël 2004, Appendix C]. Due to proximity with $Q$ - see (3-85) - there is universal constant $C$ with

$$
\left\|e^{C|y|} V_{i j}\right\|_{L^{\infty}} \rightarrow 0 \quad \text { as } b \rightarrow 0 .
$$

The following variation of $H$ is of a different nature. Set

$$
\tilde{H}(\epsilon, \epsilon)=H(\epsilon, \epsilon)-\frac{1}{\|\Lambda Q\|_{L^{2}}^{2}}\left(\epsilon_{\mathrm{re}}, L_{+} \Lambda^{2} Q\right)\left(\epsilon_{\mathrm{re}}, \Lambda Q\right),
$$

which simply alters the definition of $\mathscr{L}_{+}$given in (2-70). The following is a consequence of (2-69) and the spectral property:

Lemma 3.2 (alternative spectral property [Merle and Raphaël 2004, page 616]). There exists a universal positive constant $\tilde{\delta}_{0}<\delta_{0}$ such that, for all $\epsilon \in H^{1}$,

$$
\begin{aligned}
& \tilde{H}(\epsilon, \epsilon) \geq \tilde{\delta}_{0}\left(\int_{y \in \mathbb{R}^{2}}\left|\nabla_{y} \epsilon\right|^{2}+\int_{y \in \mathbb{R}^{2}}\left|\epsilon^{2}\right| e^{-|y|}\right)-\frac{1}{\delta_{0}} \times \\
& \left((\operatorname{Re}(\epsilon, Q))^{2}+\left(\operatorname{Re}\left(\epsilon,|y|^{2} Q\right)\right)^{2}+(\operatorname{Re}(\epsilon, y Q))^{2}+(\operatorname{Im}(\epsilon, \Lambda Q))^{2}+\left(\operatorname{Im}\left(\epsilon, \Lambda^{2} Q\right)\right)^{2}+(\operatorname{Im}(\epsilon, \nabla Q))^{2}\right) .
\end{aligned}
$$

In Lemma 3.17 and Remark 3.18 we will find that the study of linear radiation gives an accurate description of mass ejection from the singular regime. Here is a background result:

Lemma 3.3 (linear radiation [Merle and Raphaël 2004, Lemma 15]). There are universal constants $C>0$ and $\eta^{*}>0$ such that for all $0<\eta<\eta^{*}$ there is $b^{*}(\eta)>0$ such that for all $0<b<b^{*}(\eta)$ there exists a unique radial solution $\zeta_{b}$ to

$$
\left\{\begin{array}{l}
\Delta \zeta_{b}-\zeta_{b}+i b \Lambda \zeta_{b}=\Psi_{b} \\
\int\left|\nabla \zeta_{b}\right|^{2}<+\infty
\end{array}\right.
$$

where $\Psi_{b}$ is the truncation error given by (3-83); moreover, the solution satisfies the following properties, 
where we have set

$$
\Gamma_{b}=\lim _{|y| \rightarrow+\infty}|y|\left|\zeta_{b}(y)\right|^{2}:
$$

- Decay past the support of $\Psi_{b}$ :

$$
\left\||y|\left|\zeta_{b}\right|+|y|^{2}\left|\nabla \zeta_{b}\right|\right\|_{L^{\infty}\left(|y| \geq R_{b}\right)} \leq \Gamma_{b}^{\frac{1}{2}-C \eta}<+\infty
$$

- Smallness in $\dot{H}^{1}$ :

$$
\int\left|\nabla_{y} \zeta_{b}\right|^{2} \leq \Gamma_{b}^{1-C \eta}
$$

- Derivative with respect to $b$ :

$$
\left\|\frac{\partial \zeta_{b}}{\partial b}\right\|_{C^{1}} \leq \Gamma_{b}^{\frac{1}{2}-C \eta}
$$

- Stronger decay for larger $|y|$ :

$$
\begin{gathered}
\left\||y|^{2}\left|\nabla \zeta_{b}\right|\right\|_{L^{\infty}\left(|y| \geq R_{b}^{2}\right)} \leq C \frac{\Gamma_{b}^{\frac{1}{2}}}{|b|}, \\
e^{-(1+C \eta) \frac{\pi}{b}} \leq \frac{4}{5} \Gamma_{b} \leq\left\||y|^{2}\left|\zeta_{b}\right|^{2}\right\|_{L^{\infty}\left(|y| \geq R_{b}^{2}\right)} \leq e^{-(1-C \eta) \frac{\pi}{b}} .
\end{gathered}
$$

(As an estimate on $\Gamma_{b}$, (3-103) will be indispensable.)

The small universal parameter $a>0$ in the next equation will be introduced later, via (3-165), and determined on page 706, in the proof of Lemma 3.22. It influences the choice of $\eta$. We set

$$
A(t)=e^{a \frac{\pi}{b(t)}}, \quad \text { so that } \quad \Gamma_{b}^{-\frac{a}{2}} \leq A \leq \Gamma_{b}^{-\frac{3 a}{2}},
$$

and we let $\phi_{A}$ denote a radially symmetric cutoff function with

$$
\phi_{A}(y)= \begin{cases}1 & \text { for }|y| \leq A \\ 0 & \text { for }|y| \geq 2 A\end{cases}
$$

The truncated radiation $\tilde{\zeta}_{b}(y)=\phi_{A}(y) \zeta_{b}$ satisfies

$$
\Delta \tilde{\zeta}_{b}-\tilde{\zeta}_{b}+i b \Lambda \tilde{\zeta}_{b}=\Psi_{b}+F
$$

where the error term $F$ is explicit:

$$
F=\zeta_{b} \Delta \phi_{A}+2 \nabla \phi_{A} \cdot \nabla \zeta_{b}+i b \zeta_{b} y \cdot \nabla \phi_{A}
$$

In particular, by (3-102) and (3-103),

$$
|F|_{L^{\infty}}+|y \cdot \nabla F|_{L^{\infty}} \leq C \frac{\Gamma_{b}^{\frac{1}{2}}}{A} .
$$

Remark 3.4. For smaller values of $\eta$ the central profiles $\widetilde{Q}_{b}$ approximate the mass of the singular region more closely — see (3-80) — at the cost that estimates (3-85)-(3-89) are only known for ever smaller values of $b$. When $\eta$ is larger, to compensate for the imperfection of our central profile we require more 
of the radiative tail to get an accurate picture of mass transport, requiring a larger choice of $a$. See [Merle and Raphaël 2006, page 53] for similar remarks on the optimality in choice of $A(t)$.

Estimates directly due to geometric decomposition. The next lemma explains our choice of norm for $\epsilon$. Lemma 3.5 (weighted and local $L^{2}$ estimates). For any $\kappa>0$ and for all $v \in H^{1}\left(\mathbb{R}^{2}\right)$,

$$
\begin{aligned}
& \int_{y \in \mathbb{R}^{2}}|v(y)|^{2} e^{-\kappa|y|} \leq C(\kappa)\left(\int|\nabla v(y)|^{2}+\int_{|y| \leq 1}|v(y)|^{2} e^{-|y|}\right), \\
& \int_{|y| \leq \kappa}|v(y)|^{2} \leq C \kappa^{2} \log \kappa\left(\int|\nabla v(y)|^{2}+\int_{|y| \leq 1}|v(y)|^{2} e^{-|y|}\right) .
\end{aligned}
$$

Equation (3-110) is found in [Merle and Raphaël 2006, (4.11)]. While the original proof of (3-109) in [Merle and Raphaël 2004, Lemma 5] has a flaw, the methods of [Merle and Raphaël 2006] give an alternate proof.

Remark 3.6 (nonconcern for $\mu$ ). In practice, we apply these lemmas and the interaction estimates below only on regions within $\{|y| \lesssim A(t)\}$. That is, (2-55) always applies and we may choose to include measure $\mu(y)$ as appropriate.

Lemma 3.7 (estimates on interaction terms [Merle and Raphaël 2003, Section 5.3(C)]). Let $s \in\left[s_{0}, s_{1}\right.$ ), and recall from (2-73) that $\|\epsilon\|$ stands for $\int\left|\nabla_{y} \epsilon\right|^{2} \mu d y+\int_{|y| \leq \frac{10}{b}}|\epsilon|^{2} e^{-|y|} d y$.

- Estimate of first-order terms:

$$
\left|\left(\epsilon(y), P(y) \frac{d^{k}}{d y^{k}} \widetilde{Q}_{b}(y)\right)\right| \leq C(P)\|\epsilon\|^{\frac{1}{2}},
$$

where $P(y)$ is any polynomial and $0 \leq k \leq 3$.

- Estimate of second-order terms:

$$
\left|\left(R(\epsilon), P(y) \frac{d^{k}}{d y^{k}} \widetilde{Q}_{b}(y)\right)\right| \leq C(P)\|\epsilon\|,
$$

where $P(y)$ is any polynomial, $0 \leq k \leq 3$, and $R(\epsilon)$ is the terms of $\left(\epsilon+\widetilde{Q}_{b}\right)\left|\epsilon+\widetilde{Q}_{b}\right|^{2}$ formally quadratic in $\epsilon$ - see Equation (3-131).

- Estimate of localized higher-order terms:

$$
\left.\int|J(\epsilon)-| \epsilon\right|^{4} \mid \mu(y) d y \leq \delta\left(\alpha^{*}\right)\|\epsilon\|,
$$

where $J(\epsilon)-|\epsilon|^{4}=4 \operatorname{Re}\left(\epsilon|\epsilon|^{2}, \widetilde{Q}_{b}\right)$ is the term of $\left|\epsilon+\widetilde{Q}_{b}\right|^{4}$ formally cubic in $\epsilon$ and localized to the support of $\widetilde{Q}_{b}$. Similarly,

$$
\left(\widetilde{R}(\epsilon), \Lambda \widetilde{Q}_{b}\right) \leq \delta\left(\alpha^{*}\right)\|\epsilon\|,
$$

where $\widetilde{R}(\epsilon)=\epsilon|\epsilon|^{2}$ is the term of $\left(\epsilon+\widetilde{Q}_{b}\right)\left|\epsilon+\widetilde{Q}_{b}\right|^{2}$ formally cubic in $\epsilon$. 
The following estimate is our first nontrivial departure from the $L^{2}$-critical argument.

Lemma 3.8 (complete estimate on $J(\epsilon)$ ). For all $s \in\left[s_{0}, s_{1}\right)$,

$$
\int|\epsilon(y)|^{4} \mu(y) d y \leq \delta\left(\alpha^{*}\right)\|\epsilon\| \text {. }
$$

With (3-113), this gives a complete estimate for $J(\epsilon)$.

Proof. Partition the support of $\epsilon$ into two- and three- dimensional regions:

$$
\int|\epsilon(y)|^{4} \mu(y) d y=\int\left(1-\chi^{4}\right)|\epsilon(y)|^{4} \mu(y) d y+\int|\chi(\lambda y+(r, z)(s)) \epsilon(y)|^{4} \mu(y) d y .
$$

The first term on the right is supported away from $r=0$, and due to H1.1 the support of $1-\chi^{4}$ is approximately $\left\{|y|<\frac{2}{3} \frac{1}{\lambda}\right\}$, so that $\frac{1}{3} \lesssim \mu(y) \lesssim \frac{5}{3}$. We estimate this term by two-dimensional Sobolev embedding and the small mass assumption H1.2. Regarding the second term, the support of $\chi^{4}$ excludes the support of $\widetilde{Q}_{b}$ by the same reasons. Changing variables, we obtain

$$
\int|\chi(x(y)) \epsilon(y)|^{4} \mu(y) d y=\lambda^{2} \int_{x \in \mathbb{R}^{N}}|\chi(x) u(x)|^{4} d x .
$$

By the $N$-dimensional Sobolev embedding, $\dot{H}^{\frac{N}{4}} \hookrightarrow L^{4}\left(\mathbb{R}^{N}\right)$, and interpolation,

$$
\begin{aligned}
\lambda^{2} \int_{x \in \mathbb{R}^{N}}|\chi(x) u(x)|^{4} d x & \lesssim\|\chi u\|_{\dot{H}^{\frac{N}{2}-1}}^{2} \lambda^{2}\|\chi u\|_{\dot{H}^{1}\left(\mathbb{R}^{N}\right)}^{2} \\
& \lesssim\|\chi u\|_{H^{\frac{N}{2}-1}}^{2}\left(\int\left|\nabla_{y} \epsilon\right|^{2} \mu d y\right)
\end{aligned}
$$

To complete the proof, we use the assumed control H2.3 for the first and only time.

Lemma 3.9 (estimates due to conservation laws). For all $s \in\left[s_{0}, s_{1}\right)$ the following are true:

(a) Due to conservation of mass:

$$
b^{2}+\int|\tilde{u}|^{2} \lesssim\left(\alpha^{*}\right)^{\frac{1}{2}}
$$

(b) Due to conservation of energy:

$$
\begin{aligned}
2 \operatorname{Re}\left(\epsilon, \widetilde{Q}_{b}\right)-\int|\nabla \epsilon|^{2} \mu(y) d y+3 \int_{|y| \leq \frac{10}{b}} Q^{2} \epsilon_{r e}{ }^{2}+\int_{|y| \leq \frac{10}{b}} Q^{2} \epsilon_{\mathrm{im}}^{2} \\
\leq \Gamma_{b}^{1-C \eta}+\delta\left(\alpha^{*}\right)\|\epsilon\| .
\end{aligned}
$$

(c) Due to localized momentum (2-48):

$$
|\operatorname{Im}(\epsilon, \nabla \widetilde{Q})| \leq \Gamma_{b}^{2}+\delta\left(\alpha^{*}\right)\|\epsilon\|^{\frac{1}{2}} .
$$

In particular, (3-121) also holds for $\left|\left(\epsilon_{\mathrm{im}}, \operatorname{Re}\left(\nabla \widetilde{Q}_{b}\right)\right)\right|$, by Hölder and (3-85). 
Proof. (a) Conservation of mass gives $\int_{\mathbb{R}^{N}}|u(t)|^{2} d x=\int\left|u_{0}\right|^{2}$. From the geometric decomposition, expand and change some variables, obtaining

$$
\int\left|\widetilde{Q}_{b}(y)\right|^{2} \mu(y) d y+2 \operatorname{Re}\left(\int \epsilon \overline{\widetilde{Q}}_{b} \mu(y) d y\right)+\int|\tilde{u}(t)|^{2}=\int\left|u_{0}\right|^{2} .
$$

Expand the measure $\mu$. Due to the bound on $\lambda$ in (2-54), together with hypotheses H1.1 and H1.2 and the supercritical mass of $\widetilde{Q}_{b}$,

$$
\begin{aligned}
\int\left|\widetilde{Q}_{b}\right|^{2} \mu(y) d y-\int Q^{2} & =\left(\int\left|\widetilde{Q}_{b}\right|^{2}-\int Q^{2}\right)+\left(r^{N-2}(t)-1\right) \int\left|\widetilde{Q}_{b}\right|^{2}+\int \mathcal{O}\left(\lambda y_{1}\right)\left|\widetilde{Q}_{b}\right|^{2} d y \\
& \gtrsim b^{2}-\sqrt{\alpha^{*}} .
\end{aligned}
$$

Due to the smallness of $b_{0}$ and the small mass of $\epsilon_{0}$ (see C1.2), we have $\left.\left|\int_{\mathbb{R}^{N}}\right| u_{0}\right|^{2}-\int_{\mathbb{R}^{2}} Q^{2} \mid \lesssim C \alpha^{*}$. Due to local support and hypothesis H1.2, $\left|\int \epsilon \overline{\widetilde{Q}_{b}} \mu\right| \lesssim \alpha^{*}$.

(b) Conservation of energy gives $\int_{\mathbb{R}^{N}}|\nabla u(t)|^{2} d x-\frac{1}{2} \int|u|^{4}=2 E_{0}$. From the geometric decomposition,

$$
2 \lambda^{2} E_{0}=\int\left|\nabla_{y}\left(\widetilde{Q}_{b}+\epsilon\right)\right|^{2} \mu(y) d y-\frac{1}{2} \int\left|\widetilde{Q}_{b}+\epsilon\right|^{4} \mu(y) d y
$$

Partially expand the measure $\mu$ :

$$
\begin{aligned}
\int\left|\nabla_{y}\left(\widetilde{Q}_{b}+\epsilon\right)\right|^{2} \mu(y) d y=r^{N-2}(t) & \int\left|\nabla_{y} \widetilde{Q}_{b}\right|^{2}+\int \mathcal{O}\left(\lambda y_{1}\right)\left(\left|\nabla_{y} \widetilde{Q}_{b}\right|^{2}+2 \operatorname{Re}\left(\epsilon{\widetilde{\widetilde{Q}_{b}}}_{b}\right)\right) d y \\
& +2^{N-2} r(t) \operatorname{Re}\left(\int \nabla_{y} \epsilon \cdot \nabla_{y}{\widetilde{\widetilde{Q}_{b}}}_{b}\right)+\int\left|\nabla_{y} \epsilon\right|^{2} \mu(y) d y .
\end{aligned}
$$

Due to the support of $\widetilde{Q}_{b}$, the second line is of order $\lambda$, and thus inconsequential. Via a similar approach,

$$
\begin{aligned}
- & \frac{1}{2} \int\left|\widetilde{Q}_{b}+\epsilon\right|^{4} \mu(y) d y \\
=-r^{N-2}(t)\left(\frac{1}{2} \int\left|\widetilde{Q}_{b}\right|^{4}+2 \operatorname{Re}\left(\int \epsilon \widetilde{Q}_{b}\left|\widetilde{Q}_{b}\right|^{2}\right)+\right. & \left.\int|\epsilon|^{2}\left|\widetilde{Q}_{b}\right|^{2}+\operatorname{Re}\left(\int \epsilon^{2}{\widetilde{\widetilde{Q}_{b}}}^{2}\right)\right) \\
& +\lambda \odot\left(\left|\widetilde{Q}_{b}\right|^{2}\right)-\frac{1}{2} \int J(\epsilon) \mu(y) d y .
\end{aligned}
$$

Now proceed as in the $L^{2}$-critical argument. Integrate $\int \nabla_{y} \epsilon \cdot \nabla_{y}{\widetilde{Q_{b}}}_{b}$ by parts and substitute the equation for $\widetilde{Q}_{b}$ (3-83); this cancels the term of (3-126) linear in $\epsilon$. Recall the bound for $\Psi_{b}$ (3-88), the degenerate energy of $\widetilde{Q}_{b}$ (3-89), proximity to $Q$ (3-85), that $r(t) \sim 1$, and the non-trivial estimate on $J$, Equation (3-115).

(c) Our starting point is (2-48). In cylindrical coordinates, $\nabla_{x} f \cdot \nabla_{x} g=\partial_{r} f \partial_{r} g+\partial_{z} f \partial_{z} f$. For this proof we denote $r$ by $x_{1}$ and $z$ by $x_{2}$. Fix either $j=1$ or $j=2$. From the geometric decomposition,

$$
\lambda \operatorname{Im}\left(\int_{\mathbb{R}^{N}} \partial_{x_{j}} \psi^{(x)} \partial_{x_{j}} u \bar{u} d x\right)=\operatorname{Im}\left(\int \partial_{x_{j}} \psi^{(x)} \partial_{y_{j}}\left(\tilde{Q}_{b}+\epsilon\right) \overline{\left(\tilde{Q}_{b}+\epsilon\right)} \mu(y) d y\right) .
$$


Directly from definition (2-34), we have $\partial_{x_{j}} \psi^{(x)}=1$ on the support of $\widetilde{Q}_{b}$. Expand the measure $\mu$ as $r^{N-2}(t)+\mathcal{O}\left(\lambda y_{1}\right)$. Integrate by parts the interaction term in $r^{N-2}(t) \partial_{y_{j}} \epsilon \widetilde{Q}_{b}$. With the degenerate momentum of $\widetilde{Q}_{b}$ - see (3-89) - we have

$$
\begin{aligned}
2 r^{N-2}(t) \operatorname{Im}\left(\epsilon, \partial_{y_{j}} \widetilde{Q}_{b}\right) & =\operatorname{Im}\left(\int \mathcal{O}\left(\lambda y_{1}\right)\left(\partial_{y_{j}} \epsilon \overline{\widetilde{Q}_{b}}+\partial_{y_{j}} \widetilde{Q}_{b} \bar{\epsilon}+\partial_{y_{j}} \widetilde{Q}_{b} \overline{\widetilde{Q}_{b}}\right) d y\right) \\
+ & \operatorname{Im}\left(\int \partial_{x_{j}} \psi^{(x)} \partial_{y_{j}} \epsilon \bar{\epsilon} \mu(y) d y\right)-\lambda(t) \operatorname{Im}\left(\int_{\mathbb{R}^{N}} \partial_{x_{j}} \psi^{(x)} \partial_{x_{j}} u \bar{u} d x\right) .
\end{aligned}
$$

The first term on the right is of order $\lambda$, and thus negligible. For the next term we apply Hölder and the small mass assumption H1.2. The final term is controlled by H1.4.

Remark 3.10 (role of momentum conservation). The estimate analogous to (3-121) in the $L^{2}$-critical context is proven with the conservation of momentum in place of H1.4; see [Merle and Raphaël 2003, Appendix A]. As might be expected, the proof of I1.4 will resemble the proof of momentum conservation. See (3-149).

Definition 3.11 (NLS reformulated for $\epsilon$ ). For $s \in\left[s_{0}, s_{1}\right), y \in[-r(t) / \lambda(t),+\infty) \times \mathbb{R}$, and a suitable boundary condition at $y_{1}=-r(t) / \lambda(t)$, the function $\epsilon$ satisfies

$$
\begin{aligned}
i b_{s} \frac{\partial \widetilde{Q}_{b}}{\partial b}+i \epsilon_{s}-M(\epsilon)+\frac{N-2}{r\left(y_{1}\right)} \lambda \partial_{y_{1}} \epsilon & +i b \Lambda \epsilon=i\left(\frac{\lambda_{s}}{\lambda}+b\right) \Lambda \widetilde{Q}_{b}+\tilde{\gamma}_{s} \widetilde{Q}_{b}+i \frac{\left(r_{s}, z_{s}\right)}{\lambda} \cdot \nabla_{y} \widetilde{Q}_{b} \\
& +i\left(\frac{\lambda_{s}}{\lambda}+b\right) \Lambda \epsilon+\tilde{\gamma}_{s} \epsilon+i \frac{\left(r_{s}, z_{s}\right)}{\lambda} \cdot \nabla_{y} \epsilon+\Psi_{b}-R(\epsilon),
\end{aligned}
$$

where we have introduced the new variable

$$
\tilde{\gamma}(s)=-s-\gamma(s) .
$$

Note the single new term due to cylindrical symmetry. As already mentioned, the term $R(\epsilon)$ corresponds to those terms formally quadratic in $\epsilon$ :

$$
R(\epsilon)=\left(\epsilon+\widetilde{Q}_{b}\right)\left|\epsilon+\widetilde{Q}_{b}\right|^{2}-\widetilde{Q}_{b}\left|\widetilde{Q}_{b}\right|^{2}-2\left|\widetilde{Q}_{b}\right|^{2} \epsilon-\left(2 \widetilde{Q}_{b}^{2}-\operatorname{Re}\left(\widetilde{Q}_{b}^{2}\right)\right) \bar{\epsilon}
$$

Lemma 3.12 (estimates due to orthogonality conditions). For all $s \in\left[s_{0}, s_{1}\right)$,

$$
\left|\frac{\lambda_{s}}{\lambda}+b\right|+\left|b_{s}\right| \lesssim \Gamma_{b}^{1-C \eta}+\|\epsilon\|
$$

and

$$
\begin{aligned}
\left|\tilde{\gamma}_{s}-\frac{1}{|\Lambda Q|_{L^{2}}^{2}}\left(\epsilon_{r e}, L_{+}\left(\Lambda^{2} Q\right)\right)\right|+\left|\frac{r_{s}}{\lambda}\right|+\left|\frac{z_{s}}{\lambda}\right| & \leq \Gamma_{b}^{1-C \eta}+\delta\left(\alpha^{*}\right)\|\epsilon\|^{\frac{1}{2}} .
\end{aligned}
$$

Estimates (3-132) and (3-133) are a direct result of orthogonality conditions (2-39), (2-40), (2-41) and (2-42) by taking the respective inner products with $\epsilon$; see (3-129). Due to (2-54), terms resulting 
from $\frac{N-2}{r\left(y_{1}\right)} \lambda \partial_{y_{1}} \epsilon$ are inconsequential. The estimates due to energy and momentum, (3-120) and (3-121), are involved in the estimates of $\left|b_{s}\right|$ and $\left|r_{s} / \lambda\right|+\left|z_{s} / \lambda\right|$ respectively. Otherwise, all calculations are localized to the support of $\widetilde{Q}_{b}$ and are identical to the $L^{2}$-critical argument. See [Merle and Raphaël 2004, Appendix C] or [Raphaël 2005, Appendix A] for the complete calculations.

Lemma 3.13 (local virial identity). For all $s \in\left[s_{0}, s_{1}\right)$,

$$
b_{s} \geq \delta_{1}\|\epsilon\|-\Gamma_{b}^{1-C \eta},
$$

where $\delta_{1}>0$ is a universal constant and $\|\epsilon\|=\int\left|\nabla_{y} \epsilon\right|^{2} \mu d y+\int_{|y| \leq \frac{10}{b}}|\epsilon|^{2} e^{-|y|} d y$ as in (2-73).

Brief proof. Begin with the method used to prove preliminary estimate (3-132). Take the real part of the inner product of $\epsilon$ in (3-129) with $\Lambda \widetilde{Q}_{b}$. Recognize that $\partial_{s} \operatorname{Im}\left(\epsilon, \Lambda \widetilde{Q}_{b}\right)=0$ due to orthogonality condition (2-42). An adapted version of the algebraic property $L_{+}(\Lambda Q)=-2 Q$ is applied [Merle and Raphaël 2004, equation (101)]. After recognizing the equation of $\widetilde{Q}_{b}$, injecting the conservation of energy cancels the remaining terms linear in $\epsilon$. The resulting terms quadratic in $\epsilon$ are the bilinear operator $H_{b}(\epsilon, \epsilon)$ of (3-92). The remaining terms cubic in $\epsilon$ (due to the original inner product) were estimated as part of Lemma 3.7. See [Merle and Raphaël 2004, Appendix C] for the complete calculation. Controlling the auxiliary terms of the conservation of energy with (3-120) we have

$$
\begin{aligned}
-b_{s} \operatorname{Im}\left(\frac{\partial}{\partial b} \widetilde{Q}_{b}, \Lambda \widetilde{Q}_{b}\right) \gtrsim & H_{b}(\epsilon, \epsilon)+b_{s} \operatorname{Im}\left(\epsilon, \Lambda \frac{\partial}{\partial b} \widetilde{Q}_{b}\right)-\left(\frac{\lambda_{s}}{\lambda}+b\right) \operatorname{Im}\left(\epsilon, \Lambda^{2} \widetilde{Q}_{b}\right) \\
& -\tilde{\gamma}_{s} \operatorname{Re}\left(\epsilon, \Lambda \widetilde{Q}_{b}\right)-\frac{\left(r_{s}, z_{s}\right)}{\lambda} \cdot \operatorname{Im}\left(\epsilon, \nabla \widetilde{Q}_{b}\right)-\Gamma_{b}^{1-C \eta}-\delta\left(\alpha^{*}\right)\|\epsilon\| .
\end{aligned}
$$

Recall that $\partial_{b} \widetilde{Q}_{b} \approx-\frac{1}{4} i|y|^{2} Q$, make the correction (3-94) for $\widetilde{H}_{b}$, and apply the preliminary estimates (3-132) and (3-133). With the proximity to $Q$ we can write

$$
b_{s} \frac{1}{4}\|y Q\|_{L^{2}}^{2} \gtrsim H(\epsilon, \epsilon)-\tilde{\gamma}_{s}\left(\epsilon_{\mathrm{re}}, \Lambda Q\right)-\Gamma_{b}^{1-C \eta}-\delta\left(\alpha^{*}\right)\|\epsilon\| .
$$

Identify the alternate form Equation (3-95) of $\widetilde{H}$, apply the preliminary estimate for $\tilde{\gamma}_{s}$, Equation (3-133), and apply the adapted version of the spectral property, Lemma 3.2.

Remark 3.14 (progress in proving Proposition 2.8). We have already proven the first half of I1.2 as the preliminary estimate (3-119). The local virial identity with preliminary estimate (3-132) produce a closed expression for $\lambda$ and $b$, which we treat with simple arguments to prove the following lemma. In particular, (3-138) implies the first lower bound of I1.3. Following similar methods, we will then prove the second upper bound of I1.3, I1.4, I1.5, and I1.1.

Lemma 3.15 (upper bound on blowup rate). For all $s \in\left[s_{0}, s_{1}\right.$ ),

and

$$
b(s) \geq \frac{3 \pi}{4 \log s}
$$

$$
\lambda(s) \leq \sqrt{\lambda_{0}} e^{-\frac{\pi}{3} \frac{s}{\log s}} .
$$


Proof. Inject hypothesis H1.2 into the local virial identity (3-134) and carefully integrate in time. From $b>0$ and the bound on $\Gamma_{b}$ (3-103), we have

$$
\partial_{s} e^{+\frac{3 \pi}{4 b}}=-\frac{b_{s}}{b^{2}} \frac{3 \pi}{4} e^{+\frac{3 \pi}{4 b}} \leq 1, \quad \text { which implies } \quad e^{+\frac{3 \pi}{4 b}} \leq s-s_{0}+e^{+\frac{3 \pi}{4 b_{0}}} .
$$

Now (3-137) follows from our clever choice of $s_{0}$ in (2-43).

Next we view the preliminary estimate (3-132) and hypothesis H1.2 as the approximate dynamics of $\lambda$ :

$$
\left|\frac{\lambda_{s}}{\lambda}+b\right|+\left|b_{s}\right|<\Gamma_{b}^{\frac{1}{2}} \text {. }
$$

In particular, as $b>0$ is small, $-\frac{\lambda_{s}}{\lambda} \geq \frac{2 b}{3}$, which we integrate with (3-137) to get

$$
-\log \lambda \geq-\log \lambda_{0}+\int_{s_{0}}^{s} \frac{\pi}{2 \log \sigma} d \sigma .
$$

Assume $s_{0}$ is sufficiently large through the choice of data (2-29) with $\alpha^{*}$ sufficiently small, then,

$$
\int_{s_{0}}^{s} \frac{\pi}{2 \log \sigma} d \sigma \geq \frac{\pi}{3}\left(\frac{s}{\log s}-\frac{s_{0}}{\log s_{0}}\right) .
$$

From the choice of data C1.3, and (2-43), $-\log \lambda_{0} \geq e^{\frac{\pi}{2 b_{0}}}=s_{0}^{\frac{3}{2}}$. Thus

$$
-\log \lambda \geq-\frac{1}{2} \log \lambda_{0}+\frac{\pi}{3} \frac{s}{\log s},
$$

and we have proved (3-138).

A simple change of variables in (3-138) and the choice of data (2-29) and (2-32) yield a corollary:

$$
T_{\text {hyp }}=\int_{s_{0}}^{s_{1}} \lambda^{2}(\sigma) d \sigma \leq \lambda_{0} \int_{2}^{+\infty} e^{-\frac{2 \pi}{3} \frac{s}{\log s}} d s<\alpha^{*} .
$$

Proof of second upper bound in I1.3. As a direct consequence of (3-138), again assuming $s_{0}>0$ sufficiently large,

$$
-\log (s \lambda(s)) \geq \frac{\pi}{3} \frac{s}{\log s}-\log s \geq \frac{s}{\log s} .
$$

Taking the logarithm and applying (3-137),

$$
\log |-\log (s \lambda(s))| \geq \log \frac{s}{\log s} \geq \frac{4}{15} \log s \geq \frac{\pi}{5 b(s)},
$$

which leads successively to $s \lambda(s) \leq e^{-e^{\frac{\pi}{5 b}}}$ and $\lambda \leq e^{-e^{\frac{\pi}{5 b}}}$, the second upper bound of I1.3.

Proof of I1.4. Recall the approximate dynamic (3-140), which was due to the preliminary estimate (3-132) and the hypothesized control on $\epsilon$. As a consequence, for $s \in\left[s_{0}, s_{1}\right)$,

$$
\frac{d}{d s}\left(\lambda^{2} e^{\frac{5 \pi}{b}}\right)=2 \lambda^{2} e^{\frac{5 \pi}{b}}\left(\frac{\lambda_{s}}{\lambda}+b-b-\frac{5 \pi b_{s}}{2 b^{2}}\right) \leq-\lambda^{2} b e^{5 \pi} b<0,
$$


which implies

$$
\lambda^{2}(t) e^{\frac{5 \pi}{b(t)}} \leq \lambda_{0}^{2} e^{\frac{5 \pi}{b_{0}}}
$$

Then, with the estimate (3-103) on $\Gamma_{b}$, the choice of data (2-33), and the estimate on $\Gamma_{b}$ again, we obtain the energy-normalization part of I1.4:

$$
\lambda^{2}(t)\left|E_{0}\right|<\Gamma_{b(t)}^{4} e^{\frac{5 \pi}{b_{0}}} \lambda_{0}^{2}\left|E_{0}\right|<\Gamma_{b(t)}^{4} e^{\frac{5 \pi}{b_{0}}} \Gamma_{b_{0}}^{10} \ll \Gamma_{b(t)}^{4}
$$

Regarding the localized momentum, calculate directly from (1-1) that,

$$
\begin{aligned}
\frac{d}{d t} \operatorname{Im}\left(\int \nabla \psi^{(x)} \cdot \nabla u \bar{u}\right) & \\
& =\operatorname{Re}\left(\int \partial_{x_{j}} \partial_{x_{k}} \psi^{(x)} \partial_{x_{k}} u \partial_{x_{j}} \bar{u}\right)-\frac{1}{2} \int \Delta \psi^{(x)}|u|^{4}-\frac{1}{4} \int \Delta^{2} \psi^{(x)}|u|^{2} .
\end{aligned}
$$

This is a special case of the general Morawetz calculation; see, for instance, [Tao 2006, equation (3.36)]. Recall from definition (2-34) that the support of $\psi^{(x)}$ is well away from $r=0$. Apply the two-dimensional Sobolev embedding $H^{\frac{1}{2}} \hookrightarrow L^{4}$ to estimate

$$
\left|\frac{d}{d t} \operatorname{Im}\left(\int \nabla \psi^{(x)} \cdot \nabla u \bar{u}\right)\right| \leq C\left(\psi^{(x)}\right)\|u(t)\|_{H^{1}}^{2} \lesssim \frac{1}{\lambda^{2}},
$$

where the final inequality is due to hypothesized control on $\epsilon$ and the small excess mass H1.2. Note that $\int_{0}^{t} \frac{d \tau}{\lambda^{2}(\tau)}=\int_{s_{0}}^{s} d \sigma \leq s$, so we have proven

$$
\lambda(t)\left|\operatorname{Im}\left(\nabla \psi^{(x)} \cdot \nabla u(t) \bar{u}(t)\right)\right| \leq \lambda(t)\left|\operatorname{Im}\left(\nabla \psi^{(x)} \cdot \nabla u_{0} \bar{u}_{0}\right)\right|+C \lambda(t) s(t) .
$$

From the estimate (3-103) on $\Gamma_{b}$ and (3-145) from the previous proof, we have $C \lambda(t) s(t) \leq C \Gamma_{b(t)}^{10} \ll \Gamma_{b}^{4}$. Using virtually the same calculation that gave us (3-146)-(3-148) we obtain, for $s \in\left[s_{0}, s_{1}\right)$,

$$
\frac{d}{d s}\left(\lambda e^{\frac{6 \pi}{b}}\right) \leq-\frac{1}{2} \lambda b e^{\frac{6 \pi}{b}}<0
$$

and hence

$$
\lambda(t) e^{\frac{6 \pi}{b(t)}} \leq \lambda_{0} e^{\frac{6 \pi}{b_{0}}}
$$

By the estimate on $\Gamma_{b}$ and choice of data (2-33), we obtain the localized-momentum part of I1.4:

$$
\lambda(t)\left|\operatorname{Im}\left(\nabla \psi^{(x)} \cdot \nabla u_{0} \bar{u}_{0}\right)\right| \leq \Gamma_{b(t)}^{5} e^{\frac{6 \pi}{b_{0}}} \Gamma_{b_{0}}^{10} \ll \Gamma_{b(t)}^{4} .
$$

Proof of I1.5. We follow the argument found in the proof of [Raphaël 2005, Lemma 7]. Fix some $s_{2} \leq s_{3} \in\left[s_{0}, s_{1}\right)$. Substitute the local virial identity (3-134) into the preliminary estimate (3-132) to control the norm of $\epsilon$. With a crude bound for $\Gamma_{b}$,

$$
\left|\frac{\lambda_{s}}{\lambda}+b\right| \leq C\left(b_{s}+b^{2}\right)
$$


From hypothesis H1.2, $0<b^{2}<\delta\left(\alpha^{*}\right) b$ where $\delta\left(\alpha^{*}\right) \rightarrow 0$ as $\alpha^{*} \rightarrow 0$. Then,

$$
-\log \frac{\lambda\left(s_{2}\right)}{\lambda\left(s_{3}\right)}=\int_{s_{2}}^{s_{3}}\left(\frac{\lambda_{s}}{\lambda}+b\right)-\int_{s_{2}}^{s_{3}} b \leq \delta\left(\alpha^{*}\right)-\frac{1}{2} \int_{s_{2}}^{s_{3}} b \leq \delta\left(\alpha^{*}\right) .
$$

In particular, we may assume that $\alpha^{*}$ is such that $\delta\left(\alpha^{*}\right)<\log 2$, which proves I1.5.

Proof of H1.1. The preliminary estimate (3-133) can be crudely simplified to

$$
\left|\frac{r_{s}}{\lambda}\right|+\left|\frac{z_{s}}{\lambda}\right| \leq 1
$$

Then we have for all $s \in\left[s_{0}, s_{1}\right)$

$$
\left|r(s)-r_{0}\right|+\left|z(s)-z_{0}\right| \leq \int_{s_{0}}^{s}\left|r_{s}\right|+\left|z_{s}\right| \leq \int_{s_{0}}^{s} \lambda(\sigma) d \sigma \leq \sqrt{\lambda_{0}} \int_{2}^{+\infty} e^{-\frac{\pi}{3} \frac{\sigma}{\log \sigma}} d \sigma<\alpha^{*},
$$

where we applied (3-138), the choice of data (2-32) and the smallness of $b_{0}$ (2-29). With our choice of $r_{0}, z_{0}(2-28)$, this proves I1.1.

3.1. Lyapunov functional. To begin this section, we repeat the calculation of the local virial identity, this time including the linear radiation $\tilde{\zeta}_{b}$ as part of the central profile. That is, we write

$$
\tilde{\epsilon}=\epsilon-\tilde{\zeta}_{b} \Longrightarrow u(t, x)=\frac{1}{\lambda(t)}\left(\tilde{Q}_{b(t)}+\tilde{\zeta}_{b(t)}+\tilde{\epsilon}(t)\right)\left(\frac{(r, z)-(r(t), z(t))}{\lambda}\right) e^{-i \gamma(t)},
$$

where the parameters of the geometric decomposition are unchanged. The equation for $\tilde{\epsilon}$ may then be written analogously to (3-129), with a new linearized evolution operator analogous to $M$, (3-90).

Lemma 3.16 (radiative virial identity [Merle and Raphaël 2006]). For all $s \in\left[s_{0}, s_{1}\right.$ ),

$$
\partial_{s} f_{1} \geq \delta_{2}\|\tilde{\epsilon}\|+\Gamma_{b}-\frac{1}{\delta_{2}} \int_{A \leq|y| \leq 2 A}|\epsilon|^{2} d y,
$$

where $\|\tilde{\epsilon}\|=\int\left|\nabla_{y} \tilde{\epsilon}\right|^{2} \mu(y) d y+\int_{|y| \leq \frac{10}{b}}|\tilde{\epsilon}|^{2} e^{-|y|} d y$ (cf. (2-73)), $\delta_{2}, c>0$ are universal constants, and

$$
f_{1}(s)=\frac{b}{4}\left|y \widetilde{Q}_{b}\right|_{L^{2}}^{2}+\frac{1}{2} \operatorname{Im}\left(\int y \cdot \nabla \tilde{\zeta}_{b} \overline{\tilde{\zeta}}_{b}\right)+\operatorname{Im}\left(\epsilon, \Lambda \tilde{\zeta}_{b}\right)
$$

Compared with the local virial identity, the radiative virial identity is useless to control $\epsilon$ in $\dot{H}^{1}$ due to the presence of mass term $\int_{A \leq|y| \leq 2 A}|\epsilon|^{2}$. See (3-110) for further discouragement. Nevertheless, we will link this term to the ejection of mass from the singularity, through the radiation, into the dispersive regime (Lemma 3.17). Then, we will show this mass ejection is more or less uninterrupted by demonstrating the Lyapunov functional (Lemma 3.19). Finally, through conservation of energy we will prove precise bounds on the Lyapunov functional in terms of the excess mass at the singularity and $|\epsilon|_{\dot{H}^{1}}$ (Lemma 3.20). These bounds will allow us to bridge between times where $b_{s} \leq 0$ (times where the local virial identity is useful) to control $\epsilon$ pointwise in time (Lemma 3.22). 
Let $\phi_{\infty}$ be a smooth radial cutoff function on $\mathbb{R}^{2}$ satisfying

$$
\begin{gathered}
\phi_{\infty}(y)= \begin{cases}0 & \text { for }|y| \leq \frac{1}{2} \\
1 & \text { for }|y| \geq 3,\end{cases} \\
\frac{1}{4} \leq \phi_{\infty}^{\prime} \leq \frac{1}{2} \quad \text { for } 1 \leq|y| \leq 2, \quad 0 \leq \phi_{\infty}^{\prime} \quad \text { for all } y .
\end{gathered}
$$

The following lemma is proved on page 707:

Lemma 3.17 (mass ejection from singular and radiative regimes).

$$
\partial_{s}\left(\frac{1}{r^{N-2}(t)} \int \phi_{\infty}\left(\frac{y}{A}\right)|\epsilon|^{2} \mu(y) d y\right) \geq \frac{b}{400} \int_{A \leq|y| \leq 2 A}|\epsilon|^{2} d y-\Gamma_{b}^{\frac{a}{2}} \int\left|\nabla_{y} \epsilon\right|^{2} \mu(y) d y-\Gamma_{b}^{2} .
$$

Remark 3.18 (interpretation of Lemma 3.17). Assume for the sake of heuristics that $\epsilon \approx \zeta_{b}$ in the region $|y| \sim A$. With the definition of $\Gamma_{b}$ in (3-98) and the control on $\epsilon$ afforded by hypothesis H1.2, the lemma's inequality suggests continuous ejection of mass from the region $|y|<A / 2$, regardless of whether that region is growing or contracting.

Lemma 3.19 (Lyapunov functional). For all $s \in\left[s_{0}, s_{1}\right)$,

$$
\partial_{s} \mathscr{} \leq-C b\left(\Gamma_{b}+\|\tilde{\epsilon}\|+\int_{A \leq|y| \leq 2 A}|\epsilon|^{2}\right),
$$

where $C>0$ is a universal constant and

$$
\begin{aligned}
\mathscr{g}(s)=\int\left|\tilde{Q}_{b}\right|^{2}-\int|Q|^{2}+2 \operatorname{Re}\left(\epsilon, \widetilde{Q}_{b}\right) & +\frac{1}{r^{N-2}(s)} \int\left(1-\phi_{\infty}\left(\frac{y}{A}\right)\right)|\epsilon|^{2} \mu(y) d y \\
& -\frac{\delta_{2}}{800}\left(b \tilde{f}_{1}(b)-\int_{0}^{b} \tilde{f}_{1}(v) d v+b \operatorname{Im}\left(\epsilon, \Lambda \tilde{\zeta}_{b}\right)\right),
\end{aligned}
$$

$\tilde{f}_{1}$ being is the principal part of $f_{1}$ from (3-158):

$$
\tilde{f}_{1}(b)=\frac{b}{4}\left|y \widetilde{Q}_{b}\right|_{L^{2}}^{2}+\frac{1}{2} \operatorname{Im}\left(\int y \cdot \nabla \tilde{\zeta}_{b} \overline{\tilde{\zeta}}_{b}\right)
$$

The proof, which we defer until page 708, involves the radiative virial estimate (3-157), the mass dispersion estimate in Lemma 3.17, and conservation of mass.

Now let us discuss what $\mathscr{g}$ is.

Lemma 3.20 (estimates on Lyapunov functional). For all $s \in\left[s_{0}, s_{1}\right.$ ) we have the crude estimate

$$
\left|\mathscr{}-d_{0} b^{2}\right|<\delta_{3} b^{2}
$$

where $0<\delta_{3} \ll 1$ is a universal constant and $d_{0} b^{2}$ is the approximate excess mass of the profile $\widetilde{Q}_{b}$ (see (3-87)). There also holds a more refined estimate:

$$
-\Gamma_{b}^{1-C a}+\frac{1}{C}\|\epsilon\| \leq \mathscr{g}(s)-f_{2}(b(s)) \leq \Gamma_{b}^{1-C a}+C A^{2}\|\epsilon\|,
$$


where $f_{2}$ is the principal part of $\mathscr{F}$ concerned with the mass of the profile:

$$
f_{2}(b)=\int\left|\widetilde{Q}_{b}\right|^{2}-\int|Q|^{2}-\frac{\delta_{2}}{800}\left(b \tilde{f}_{1}(b)-\int_{0}^{b} \tilde{f}_{1}(v) d v\right) .
$$

Proof. To prove (3-164) we will approximate each term of (3-162). To estimate the term in $|\epsilon|^{2}$, recall from (3-159) the support of $\phi_{\infty}$, and derive the consequence for $\mu(y)$ similar to Equation (2-55). One obtains

$$
\int\left(1-\phi_{\infty}\left(\frac{y}{A}\right)\right)|\epsilon|^{2} \mu(y) d y \lesssim \int_{|y| \leq 3 A}|\epsilon|^{2} \lesssim A^{2} \log A\|\epsilon\| \leq \Gamma_{b}^{\frac{1}{2}},
$$

where the second inequality is due to Lemma 3.5 and the final inequality is from the definition (3-104) of $A$ and the hypothesized control of $\epsilon$. Estimate $\left(\epsilon, \widetilde{Q}_{b}\right.$ ) by the same control, and the terms in $\tilde{\zeta}_{b}$ by (3-100). Equation (3-164) then follows from (3-87) by noting that the constant $\delta_{2}$ due to the radiative virial identity (3-157) can be assumed small with respect to universal constant $d_{0}$, so that $0<\left.\left(\partial f_{2} / \partial b^{2}\right)\right|_{b^{2}=0}<\infty$.

Next we prove the refined estimate. Note that

$$
\mathscr{g}(s)-f_{2}(b(s))=2 \operatorname{Re}\left(\epsilon, \widetilde{Q}_{b}\right)+\frac{1}{r^{N-2}(t)} \int\left(1-\phi_{\infty}\right)|\epsilon|^{2} \mu(y)-\frac{\delta_{2}}{800} b \operatorname{Im}\left(\epsilon, \Lambda \tilde{\zeta}_{b}\right) .
$$

By the bounds for $\tilde{\zeta}_{b}$ in Lemma 3.5 and the choice of $A$, we have

$$
\left|\operatorname{Im}\left(\epsilon, \Lambda \tilde{\zeta}_{b}\right)\right| \leq \Gamma_{b}^{\frac{1}{2}-C \eta}\left(\int_{|y| \leq A}|\epsilon|^{2}\right)^{\frac{1}{2}} \lesssim \Gamma_{b}^{\frac{1}{2}-C \eta} A(\log A)^{\frac{1}{2}}\|\epsilon\|^{\frac{1}{2}} \lesssim \Gamma_{b}^{1-C a}+\|\epsilon\| .
$$

Since $b$ is small, the contribution of (3-169) is a factor of $\alpha^{*}$ smaller than the desired bound. Similar terms will be omitted for the remainder of the proof.

Regarding the two other terms in (3-168), the term linear in $\epsilon$ we recognize from the conservation of energy (3-120). Indeed, the upper bound for (3-168) follows from (3-120) with (3-109) and

$$
\int\left(1-\phi_{\infty}\right)|\epsilon|^{2} \mu(y) d y \lesssim A^{2} \log A\|\epsilon\|,
$$

which is due to (3-110).

To establish a lower bound for (3-168) we will need the following lemma, whose proof is based on a spectral result due to [Martel and Merle 2001], with additional properties proven in [Mariş 2002] and [McLeod 1993]. See [Merle and Raphaël 2006, Lemma 8] for that spectral property, and Appendix D of the same reference for a proof of the lemma.

Lemma 3.21 (elliptic estimate for $L$ ). Recall the linearized Schrödinger operator $L$ from (2-68). There exists a universal constant $\delta_{4}>0$ such that, for all $v \in H^{1}\left(\mathbb{R}^{2}\right)$,

$$
\begin{aligned}
& \operatorname{Re}(L(v), v)-\int \phi_{\infty}|v|^{2} \\
& \quad \geq \delta_{4}\left(\int|\nabla v|^{2}+\int|v|^{2} e^{-|y|}\right)-\frac{1}{\delta_{4}}\left(\operatorname{Re}(v, Q)+\operatorname{Re}\left(v,|y|^{2} Q\right)+\operatorname{Re}(v, y Q)+\operatorname{Im}\left(v, \Lambda^{2} Q\right)\right)^{2} .
\end{aligned}
$$


Introduce a new radially symmetric cutoff function, analogous to $\phi_{A}$ (3-105) but with larger support, such that $\left(1-\phi_{B}(y)\right)\left(1-\phi_{\infty}(y / A)\right)=0$ :

$$
\phi_{B}(y)= \begin{cases}1 & \text { for }|y| \leq 3 A \\ 0 & \text { for }|y| \geq 4 A\end{cases}
$$

By (2-55), we can rewrite the principal part of the conservation of energy estimate (3-120) as

$$
2 \operatorname{Re}\left(\epsilon, \tilde{Q}_{b}\right) \approx \int\left(1-\phi_{B}^{2}\right)|\nabla \epsilon|^{2} \mu(y) d y+\int \phi_{B}^{2}|\nabla \epsilon|^{2} d y-3 \int Q^{2}\left(\phi_{B} \epsilon_{\mathrm{re}}\right)^{2}-\int Q^{2}\left(\phi_{B} \epsilon_{\mathrm{im}}\right)^{2},
$$

where we used the exponential spatial decay of $Q$ and the lower bound for $\Gamma_{b}$ (3-98) to control the excess in $Q^{2} \epsilon^{2}$ on $|y|>\frac{10}{b}$. With integration by parts,

$$
\int \phi_{B}^{2}|\nabla \epsilon|^{2} d y=\int\left|\nabla\left(\phi_{B} \epsilon\right)\right|^{2} d y+\int \Delta \phi_{B} \phi_{B}|\epsilon|^{2} d y .
$$

The principal part of (3-168) is then

$$
\begin{aligned}
& 2 \operatorname{Re}\left(\epsilon, \widetilde{Q}_{b}\right)+\frac{1}{r^{N-2}(t)} \int\left(1-\phi_{\infty}\right)|\epsilon|^{2} \mu(y) d y \approx \int\left(1-\phi_{B}^{2}\right)|\nabla \epsilon|^{2} \mu(y) d y \\
& +\left(\operatorname{Re}\left(L\left(\phi_{B} \epsilon\right), \phi_{B} \epsilon\right)-\int \phi_{\infty}\left|\phi_{B} \epsilon\right|^{2}\right)+\int \Delta \phi_{B} \phi_{B}|\epsilon|^{2}+\int\left(1-\phi_{\infty}\right)\left(\frac{\mu}{r^{N-2}(t)}-\phi_{B}^{2}\right)|\epsilon|^{2} .
\end{aligned}
$$

The final term can be neglected, since $\left(1-\phi_{\infty}\right)\left(\mu / r^{N-2}(t)-\phi_{B}^{2}\right)$ is of order $\lambda y_{1}$, and supported on $|y|<4 A$. The lower bound for (3-168) then follows from Lemma 3.21, an integration by parts, and the straightforward comparison,

$$
\int \phi_{B}^{2}|\nabla \epsilon|^{2}+\int\left|\phi_{B} \epsilon\right|^{2} e^{-|y|} \gtrsim \int \phi_{B}^{2}|\nabla \epsilon|^{2} \mu(y)+\int_{|y| \leq \frac{10}{b}}|\epsilon|^{2} e^{-|y|},
$$

again due to the support of $\phi_{B}$ and the bound on $\lambda$. This completes the proof of (3-165).

Lemma 3.22 (lower bound on blowup rate). For all $s \in\left[s_{0}, s_{1}\right)$,

and

$$
b(s) \leq \frac{4 \pi}{3 \log s}
$$

$$
\int_{s_{0}}^{s}\left(\Gamma_{b(\sigma)}+\|\epsilon\|\right) d \sigma \leq C \alpha^{*},
$$

where $C>0$ is a universal constant and

$$
\|\epsilon\| \leq \Gamma_{b}^{\frac{4}{5}} .
$$

(This is (2-58), the remaining part of I1.2.)

Note that (3-177) is the first upper bound of I1.3. The only estimate still required in order to establish Proposition 2.8 follows as a corollary. 
Proof of the second lower bound I1.3. Recall from (3-140) the approximate dynamics of $\lambda$. Since $b>0$ is small, we have $-\frac{\lambda_{s}}{\lambda} \leq 3 b$, which we integrate with (3-177),

$$
-\log \lambda(s) \leq-\log \lambda_{0}+4 \pi \int_{s_{0}}^{s} \frac{1}{\log \sigma} d \sigma \leq-\log \lambda_{0}+4 \pi\left(s-s_{0}\right) .
$$

Use (3-177) again, and recall the definition of $s_{0}$ (2-43) and choice of data (2-32), to obtain

$$
\lambda(s) \geq \lambda_{0} e^{4 \pi s_{0}} e^{-4 \pi e^{\frac{4 \pi}{3 b(s)}}}>e^{-e^{\frac{5 \pi}{b(s)}}} .
$$

Proof of Lemma 3.22. First, in view of the crude estimate (3-164), we may divide the Lyapunov inequality (3-161) by $\sqrt{\mathscr{g}}$ and integrate in time, leaving

$$
\int_{s_{0}}^{s}\left(\Gamma_{b(\sigma)}+\|\epsilon\|\right) d \sigma \leq C\left(\sqrt{\mathscr{g}\left(s_{0}\right)}-\sqrt{\mathscr{g}(s)}\right) \leq C b_{0} .
$$

The choice of data (2-29) then proves (3-178). Alternately, we may view the crude estimate (3-164) and the Lyapunov inequality (3-161) as giving a differential inequality for $\mathscr{F}$ :

$$
\partial_{s} e^{+\frac{5 \pi}{4} \sqrt{\frac{d_{0}}{g}}} \gtrsim \frac{b}{\mathscr{g}} \Gamma_{b} e^{\frac{5 \pi}{4} \sqrt{\frac{d_{0}}{g}}} \geq 1,
$$

which implies

$$
e^{+\frac{5 \pi}{4} \sqrt{\frac{d_{0}}{\mathscr{\Psi}(s)}}} \geq e^{+\frac{5 \pi}{4} \sqrt{\frac{d_{0}}{\Phi\left(s_{0}\right)}}}+s-s_{0} .
$$

Here we applied the bound (3-103) on $\Gamma_{b}$, for which it is essential that $\frac{5}{4}>1+C \eta$; see Remark 4.15. By the crude estimate (3-164) and the definition of $s_{0}$ in (2-43), we have

$$
e^{+\frac{5 \pi}{4} \sqrt{\frac{d_{0}}{\Phi\left(s_{0}\right)}}}>e^{\frac{\pi}{b_{0}}}>s_{0},
$$

which, again with estimate (3-164), proves (3-177) from (3-183).

It remains to establish the pointwise control of $\epsilon$. Fix $s \in\left[s_{0}, s_{1}\right)$.

1. If $\partial_{s} b(s) \leq 0$, then (3-179) follows from the local virial identity, Lemma 3.13.

2. If $\partial_{s} b(s)>0$, there exists a largest interval $\left(s_{+}, s\right)$, with $s_{0} \leq s_{+}$, on which $\partial_{s} b>0$. This implies $b\left(s_{+}\right)<b(s)$ and either

$$
s_{+}=s_{0} \quad \text { or } \quad \partial_{s} b\left(s_{+}\right)=0 .
$$

In the first case we use the choice of small $\epsilon_{0}$ and in the second the local virial identity, to obtain in either case

$$
\int\left|\nabla_{y} \epsilon\left(s_{+}, y\right)\right|^{2} \mu(y) d y+\int_{|y| \leq \frac{10}{b\left(s_{+}\right)}}\left|\epsilon\left(s_{+}, y\right)\right|^{2} e^{-|y|} d y \leq \Gamma_{b\left(s_{+}\right)}^{\frac{6}{7}} .
$$

From the upper bound of the refined estimate (3-165), and assuming $a>0$ is sufficiently small,

$$
\mathscr{f}\left(s_{+}\right)-f_{2}\left(b\left(s_{+}\right)\right) \leq \Gamma_{b\left(s_{+}\right)}^{\frac{5}{6}}<\Gamma_{b(s)}^{\frac{5}{6}} .
$$


Since $\mathscr{F}$ is non-increasing, and from the lower bound of refined estimate (3-165), we get

$$
\begin{aligned}
\Gamma_{b(s)}^{\frac{5}{6}} & \geq \mathscr{g}(s)-f_{2}\left(b\left(s_{+}\right)\right) \\
& \gtrsim\left(\int\left|\nabla_{y} \epsilon(s, y)\right|^{2} \mu(y) d y+\int_{|y| \leq \frac{10}{b(s)}}|\epsilon(s, y)|^{2} e^{-|y|} d y\right)-\Gamma_{b(s)}^{1-C a}+\left(f_{2}(b(s))-f_{2}\left(b\left(s_{+}\right)\right)\right)
\end{aligned}
$$

As noted in the proof of the crude estimate (3-164), we may assume the constant $\delta_{2}$ of (3-157) is small enough relative to $d_{0}$ so that $0<\partial f_{2} /\left.\partial_{b^{2}}\right|_{b^{2}=0}<\infty$, proving that $\left(f_{2}(b(s))-f_{2}\left(b\left(s_{+}\right)\right)\right)>0$. Assuming $a>0$ is sufficiently small, this proves (3-179).

Proof of Lemma 3.17. Directly from (1-1) we obtain

$$
\begin{array}{r}
\frac{1}{2} \partial_{s}\left(\int \phi_{\infty}\left(\frac{(r, z)-(r(t), z(t))}{\lambda A}\right)|u|^{2} d x\right)=\frac{1}{\lambda A} \operatorname{Im}\left(\int \nabla_{x} \phi_{\infty}\left(\frac{y}{A}\right) \cdot \nabla_{x} u \bar{u} d x\right) \\
-\frac{1}{2 \lambda^{2} A} \int\left(\left(\frac{\lambda_{s}}{\lambda}+\frac{A_{s}}{A}\right) y+\frac{\partial_{s}(r, z)}{\lambda}\right) \cdot \nabla_{x} \phi_{\infty}\left(\frac{y}{A}\right)|u|^{2} d x .
\end{array}
$$

By the choice of $A$ in (3-104) and the properties of $\phi_{\infty}$ in (3-159), the support of $\widetilde{Q}_{b}$ and $\phi_{\infty}(y / A)$ are disjoint. With the geometric decomposition and a change of variables we can rewrite (3-187) in terms of $|\epsilon|^{2}$ :

$$
\begin{aligned}
\frac{1}{2} \frac{d}{d s} \int \phi_{\infty} & \left(\frac{y}{A}\right)|\epsilon|^{2} \mu(y) d y \\
= & \frac{1}{A} \operatorname{Im}\left(\int \nabla_{x} \phi_{\infty}\left(\frac{y}{A}\right) \cdot \nabla_{y} \epsilon \bar{\epsilon} \mu(y) d y\right)+\frac{b}{2} \int \frac{y}{A} \cdot \nabla_{x} \phi_{\infty}\left(\frac{y}{A}\right)|\epsilon|^{2} \mu(y) d y \\
& \quad-\frac{1}{2 A} \int\left(\left(\frac{\lambda_{s}}{\lambda}+b+\frac{A_{s}}{A}\right) y+\frac{\partial_{s}(r, z)}{\lambda}\right) \cdot \nabla_{x} \phi_{\infty}\left(\frac{y}{A}\right)|\epsilon|^{2} \mu(y) d y .
\end{aligned}
$$

By Cauchy-Schwarz, the definition of $A$ in (3-104) and the lower bound on $\Gamma_{b}$ in (3-103),

$$
\begin{aligned}
\mid \frac{1}{A} \operatorname{Im}\left(\int \nabla_{x} \phi_{\infty}\left(\frac{y}{A}\right) \cdot \nabla_{y} \epsilon\right. & \bar{\epsilon} \\
& \mu(y) d y) \mid \\
& \leq \frac{1}{A}\left(\int|\nabla \epsilon|^{2} \mu(y) d y\right)^{\frac{1}{2}}\left(\int\left|\nabla_{x} \phi_{\infty}\left(\frac{y}{A}\right)\right||\epsilon|^{2} \mu(y) d y\right)^{\frac{1}{2}} \\
& \leq \frac{1}{2} \Gamma_{b}^{\frac{a}{2}} \int|\nabla \epsilon|^{2} \mu(y) d y+\frac{b}{40} \int\left|\nabla_{x} \phi_{\infty}\left(\frac{y}{A}\right)\right||\epsilon|^{2} \mu(y) d y .
\end{aligned}
$$

The factor $\frac{b}{40}$ is arbitrary by assuming $b$ is sufficiently small. The following term is the principal part of (3-188). From Equation (3-160) we know the support of $\phi_{\infty}^{\prime}$ and that $\phi_{\infty}^{\prime} \geq 0$, so

$$
\frac{b}{2} \int \frac{y}{A} \cdot \nabla_{x} \phi_{\infty}\left(\frac{y}{A}\right)|\epsilon|^{2} \mu(y) d y \geq \frac{b}{5} \int\left|\nabla_{x} \phi_{\infty}\left(\frac{y}{A}\right)\right||\epsilon|^{2} \mu(y) d y .
$$


Regarding the last line of (3-188), apply preliminary estimates (3-132) and (3-133), the support of $\phi_{\infty}^{\prime}$, and the definition of $A$ to estimate

$$
\frac{1}{2 A}\left|\left(\frac{\lambda_{s}}{\lambda}+b+\frac{A_{s}}{A}\right) y+\frac{\partial_{s}(r, z)}{\lambda}\right| \leq \frac{b}{40} .
$$

Due to the bounds for $\phi_{\infty}^{\prime}(y / A)$ on $A \leq|y| \leq 2 A$, and lower bounds for $\mu$ similar to (2-55), we have

$$
\int\left|\nabla_{x} \phi_{\infty}\left(\frac{y}{A}\right)\right||\epsilon|^{2} \mu(y) d y \geq \frac{1}{6} \int_{A \leq|y| \leq 2 A}|\epsilon|^{2} d y .
$$

From (3-188) we have proven,

$$
\frac{d}{d s} \int \phi_{\infty}\left(\frac{y}{A}\right)|\epsilon|^{2} \mu(y) d y \geq \frac{b}{20} \int_{A \leq|y| \leq 2 A}|\epsilon|^{2} d y-\Gamma_{b}^{\frac{a}{2}} \int|\nabla \epsilon|^{2} \mu(y) d y .
$$

Finally note that by the preliminary estimate (3-133), the fact that $r(t) \sim 1$ from H1.1, a change of variables, and the log-log relationship (2-54), we have the easy estimate

$$
\left.\left.\left|\frac{r_{s}}{r^{N-1}(t)} \int \phi_{\infty}\left(\frac{y}{A}\right)\right| \epsilon\right|^{2} \mu(y) d y\left|\ll \lambda \int\right| \tilde{u}\right|^{2} \ll \Gamma_{b}^{2} .
$$

This completes the proof of Lemma 3.17.

Proof of Lemma 3.19. We multiply the radiative virial identity (3-157) by $\frac{\delta_{2} b}{800}$ and sum with the mass ejection estimate in Lemma 3.17 to cancel the bad sign of $\int_{A \leq|y| \leq 2 A}|\epsilon|^{2}$ :

$$
\begin{aligned}
\partial_{s}\left(\frac{1}{r^{N-2}(t)} \int \phi_{\infty}\right. & \left.\left(\frac{y}{A}\right)|\epsilon|^{2} \mu(y) d y\right)+\frac{\delta_{2} b}{800} \partial_{s} f_{1} \\
& \geq \frac{\delta_{2}^{2} b}{800}\|\tilde{\epsilon}\|+\frac{b}{800} \int_{A \leq|y| \leq 2 A}|\epsilon|^{2} d y+\frac{\delta_{2} b}{1000} \Gamma_{b}-\Gamma_{b}^{\frac{a}{2}} \int\left|\nabla_{y} \epsilon\right|^{2} \mu(y) d y .
\end{aligned}
$$

The final term of (3-195) has the bad sign. Recall from (3-156) that $\epsilon=\tilde{\epsilon}+\tilde{\zeta}_{b}$, and from (3-100) that $\tilde{\zeta}_{b}$ is small in $\dot{H}^{1}$, on the support of which we can estimate $\mu$, so that

$$
\begin{aligned}
\Gamma_{b}^{\frac{a}{2}} \int\left|\nabla_{y} \epsilon\right|^{2} \mu(y) d y & \lesssim \Gamma_{b}^{\frac{a}{2}}\left(\Gamma_{b}^{1-C \eta}+\int|\nabla \tilde{\epsilon}|^{2} \mu(y) d y\right) \\
& \leq \Gamma_{b}^{1+\frac{a}{4}}+\Gamma_{b}^{\frac{a}{2}} \int|\nabla \tilde{\epsilon}|^{2} \mu(y) d y,
\end{aligned}
$$

where for the second inequality we require $a>4 C \eta$; see Remark 3.4. To rewrite $\frac{\delta_{2} b}{800} \partial_{s} f_{1}$, note that

$$
b \partial_{s} f_{1}=\partial_{s}\left(b \tilde{f}_{1}(b)-\int_{0}^{b} \tilde{f}_{1}(v) d v+b \operatorname{Im}\left(\epsilon, \Lambda \tilde{\zeta}_{b}\right)\right)-\partial_{s} b \operatorname{Im}\left(\epsilon, \Lambda \tilde{\zeta}_{b}\right),
$$

where $\tilde{f}_{1}$ is the principal part of $f_{1}$; see (3-163) and (3-158), respectively. Estimate the final term of (3-197) with a combination of the preliminary estimate (3-132), Hölder, Lemma 3.5, and H1.2. 
Equation (3-195) is transformed into

$$
\begin{aligned}
\partial_{s}\left(\frac{1}{r^{N-2}(t)} \int \phi_{\infty}\left(\frac{y}{A}\right)|\epsilon|^{2} \mu(y) d y+\frac{\delta_{2}}{800}\right. & \left.\left(b \tilde{f}_{1}(b)-\int_{0}^{b} \tilde{f}_{1}(v) d v+b \operatorname{Im}\left(\epsilon, \Lambda \tilde{\zeta}_{b}\right)\right)\right) \\
& \geq \frac{\delta_{2}^{2} b}{800}\left(\|\tilde{\epsilon}\|+\int_{A \leq|y| \leq 2 A}|\epsilon|^{2} d y\right)+\frac{\delta_{2} b}{2000} \Gamma_{b} .
\end{aligned}
$$

To identify the left-hand side of (3-198) with $-\partial_{s} \mathscr{E}$, inject the conservation of mass, $\int_{\mathbb{R}^{N}}|u(t)|^{2}=$ $\int\left|u_{0}\right|^{2}$. As we did for Equation (3-122), rewrite $u(t)$ with the geometric decomposition, expand the product, change variables, expand the measure $\mu$, divide by $r^{N-2}(t)$, and take the derivative $\partial_{s}$ :

$$
\begin{aligned}
\partial_{s}\left(\frac{1}{r^{N-2}(t)} \int \phi_{\infty}\left(\frac{y}{A}\right)|\epsilon|^{2} \mu(y) d y\right)=-\partial_{s}\left(\int\left|\widetilde{Q}_{b}\right|^{2}-\int|Q|^{2}+2 \operatorname{Re}\left(\epsilon, \widetilde{Q}_{b}\right)\right) \\
-\partial_{s}\left(\frac{1}{r^{N-2}(t)} \int \mathcal{O}\left(\lambda y_{1}\right)\left(\left|\widetilde{Q}_{b}\right|^{2}+2 \operatorname{Re}\left(\epsilon \overline{\widetilde{Q}_{b}}\right)\right)\right)-\frac{\partial_{s} r}{r^{N-1}(t)} \int\left|u_{0}\right|^{2} .
\end{aligned}
$$

Through a combination of the preliminary estimates (3-132) and (3-133), the $\epsilon$-equation (3-129), and the $\log$-log rate (2-54), we obtain

$$
\left|-\partial_{s}\left(\frac{1}{r^{N-2}(t)} \int \mathcal{O}\left(\lambda y_{1}\right)\left(\left|\widetilde{Q}_{b}\right|^{2}+2 \operatorname{Re}\left(\epsilon \overline{\widetilde{Q}_{b}}\right)\right)\right)\right| \lesssim \lambda<\Gamma_{b}^{2} .
$$

Likewise,

$$
\left|\frac{\partial_{s} r}{r^{2}(t)}\right| \int\left|u_{0}\right|^{2} \lesssim \lambda \int\left|u_{0}\right|^{2}<\Gamma_{b}^{2}
$$

Inserting (3-199) into (3-198) completes the proof of Lemma 3.19.

\section{Proof of global behavior}

In this section we prove that the properties I2.1-I2.3 follow from hypotheses H1.1-H2.3. The following properties of the singular dynamic proven in Section 3 will be used: the specific log-log rate, the geometric decomposition and resulting control on $b_{s}$, and the integrability of $\|\tilde{u}\|_{L_{t}^{2} H_{x}^{1}}$.

Growth of $\|\boldsymbol{u}\|_{\boldsymbol{H}^{\boldsymbol{N}}}$. It is left until Section 5 to show that $1 / \lambda$ follows the log-log rate (1-12). Here, we use the log-log rate in the form H1.3, and the control of $b_{s}$, to prove directly that $\lambda^{-1}(t)$ has the same integrability in time as

$$
\sqrt{\frac{\log |\log (T-t)|}{T-t}}
$$

Lemma 4.1 (Integrability due to log-log rate[Raphaël and Szeftel 2009, (51)]). Let $0 \leq \mu<2$ and $\sigma_{1} \in \mathbb{R}$. Then,

$$
\int_{0}^{t} \frac{e^{\frac{\sigma_{1}}{b(\tau)}}}{\lambda^{\mu}(\tau)} d \tau \lesssim C\left(\mu, \sigma_{1}, \alpha^{*}\right)
$$

where for fixed $\mu$ and $\sigma_{1}, C\left(\mu, \sigma_{1}, \alpha^{*}\right)$ decays much faster than $e^{-\frac{1}{\alpha^{*}}}$ as $\alpha^{*} \rightarrow 0$. 
Proof. The $\log -\log$ rate, H1.3, gives $e^{\frac{\pi}{10 b}}<|\log \lambda|$ and $\frac{\pi}{10 b}>\frac{1}{100} \log s$; hence

$$
\frac{1}{\lambda}>e^{+e^{\frac{\pi}{10 b}}}>e^{\frac{1}{100}}
$$

By a change of variables and the almost-monotony of $\lambda, \mathrm{H} 1.5$,

$$
\int_{0}^{t} \frac{e^{\frac{\sigma_{1}}{b(\tau)}}}{\lambda^{\mu}(\tau)} d \tau<\int_{s_{0}}^{s} \frac{|\log \lambda|^{\frac{10 \sigma_{1}}{\pi}}}{\lambda^{\mu-2}\left(\tau^{\prime}\right)} d \tau^{\prime} \lesssim \frac{|\log \lambda(t)|^{\frac{10 \sigma_{1}}{\pi}}}{\lambda^{\mu-2}(t)}\left(s(t)-s_{0}\right) \lesssim e^{(\mu-2) s \frac{1}{100}(t) .}
$$

Finally, to prove the behavior of $C\left(\mu, \sigma_{1}, \alpha^{*}\right)$, recall that $s(t) \geq s_{0}=e^{\frac{3 \pi}{4 b_{0}}}$ and $b_{0}<\alpha^{*}$.

Remark 4.2 (Lemma 4.1 for $\mu \geq 2$ ). From the $\log \log$ rate, H1.3, $s(t)-s_{0} \lesssim e^{\frac{10}{\pi} \frac{1}{b(t)}}$, so by the same proof,

$$
\int_{0}^{t} \frac{1}{\lambda^{\mu}} \lesssim \frac{e^{\frac{10}{\pi} \frac{1}{b(t)}}}{\lambda^{\mu-2}}
$$

This is the primary integrability tool of [Raphaël and Szeftel 2009]. The following improvement will be crucial.

Lemma 4.3 (refined integrability due to control of $b_{s}$ ). Let $\mu>2$ and $\sigma^{*}$ be arbitrary and assume $\alpha^{*}>0$ is sufficiently small. Then for any $\sigma_{2}>0$ and all $t \in\left[0, T_{\mathrm{hyp}}\right)$,

$$
\int_{0}^{t} \frac{e^{-\frac{\sigma^{*}}{b(\tau)}}}{\lambda^{\mu}(\tau)} d \tau \leq C\left(\mu, \sigma_{2}, \alpha^{*}\right) \frac{e^{-\frac{\sigma^{*}}{b(t)}} e^{+\frac{\sigma_{2}}{b(t)}}}{\lambda^{\mu-2}(t)}
$$

where, for fixed $\mu$ and $\sigma_{2}, C\left(\mu, \sigma_{2}, \alpha^{*}\right) \rightarrow 0$ as $\alpha^{*} \rightarrow 0$.

Proof. To begin, we prove the case $\sigma^{*}=0$. By direct calculation,

$$
\frac{d}{d s}\left(\frac{1}{b} \frac{1}{\lambda^{\mu-2}}\right)=\frac{1}{\lambda^{\mu-2}}\left((\mu-2)-\frac{b_{s}}{b^{2}}-(\mu-2) \frac{\frac{\lambda_{s}}{\lambda}+b}{b}\right) .
$$

For $\alpha^{*}$ sufficiently small relative to $\mu$, from $\mathrm{H} 1.2$ and the control of $b_{s}$, (3-132),

$$
\frac{1}{\lambda^{\mu}} \leq C(\mu) \frac{1}{\lambda^{2}} \frac{d}{d s}\left(\frac{1}{b} \frac{1}{\lambda^{\mu-2}}\right)=C(\mu) \frac{d}{d t}\left(\frac{1}{b} \frac{1}{\lambda^{\mu-2}}\right) .
$$

After integration, we estimate

$$
C(\mu) \frac{1}{b} \frac{1}{\lambda^{\mu-2}} \leq C\left(\mu, \sigma_{2}, \alpha^{*}\right) \frac{e^{+\frac{\sigma_{2}}{b}}}{\lambda^{\mu-2}} .
$$

For those cases where $\sigma^{*} \neq 0$, integrate by parts:

$$
\int_{0}^{t} \frac{e^{-\frac{\sigma^{*}}{b(\tau)}}}{\lambda^{\mu}(\tau)} d \tau=\left.e^{-\frac{\sigma^{*}}{b(\tau)}} \int_{0}^{\tau} \frac{1}{\lambda^{\mu}\left(\tau^{\prime}\right)} d \tau^{\prime}\right|_{0} ^{t}-\int_{0}^{t} \sigma^{*}\left(\frac{b_{\tau}}{b^{2}} e^{-\frac{\sigma^{*}}{b(\tau)}} \int_{0}^{\tau} \frac{1}{\lambda^{\mu}\left(\tau^{\prime}\right)} d \tau^{\prime}\right) d \tau .
$$

Apply the previous case to the first term on the right. For the second term, make the change of variable $b_{\tau}=b_{s(\tau)} / \lambda^{2}(\tau)$ and apply the previous case for some $\sigma_{2} \ll \frac{1}{2}$. Use (3-132) to approximate $b_{s}$, and we have bounded the second term by a small multiple of the left-hand side. 
Lemma 4.3 is not true for $\mu=2$. As a substitute, we prove a corollary of the integrated Lyapunov inequality, (3-178).

Corollary 4.4. Let $\sigma_{3} \geq 0$. For all $t \in\left[0, T_{\text {hyp }}\right)$,

$$
\int_{0}^{t} e^{\frac{\sigma_{3}}{b(\tau)}}\left(\|\tilde{u}(\tau)\|_{H^{1}}^{2}+\frac{\Gamma_{b(\tau)}}{\lambda^{2}(\tau)}\right) d \tau \lesssim C\left(\alpha^{*}\right) e^{\frac{\sigma_{3}}{b(t)}}
$$

Proof. By change of variables and integration by parts,

$$
\begin{aligned}
& \int_{0}^{t} e^{\frac{\sigma_{3}}{b(\tau)}}\left(\|\tilde{u}(\tau)\|_{H^{1}}^{2}+\frac{\Gamma_{b(\tau)}}{\lambda^{2}(\tau)}\right) d \tau \\
& \quad=e^{\frac{\sigma_{3}}{b(\sigma)}} \int_{0}^{\sigma} \lambda^{2}\left(\sigma^{\prime}\right)\left\|\tilde{u}\left(\sigma^{\prime}\right)\right\|_{H_{x}^{1}}^{2}+\left.\Gamma_{b\left(\sigma^{\prime}\right)} d \sigma^{\prime}\right|_{s_{0}} ^{s(t)}+\sigma_{3} \int_{s_{0}}^{s(t)} \frac{b_{s}}{b^{2}} e^{\frac{\sigma_{3}}{b(\sigma)}}\left(\int_{0}^{\sigma} \lambda^{2}\|\tilde{u}\|_{H_{x}^{1}}^{2}+\Gamma_{b}\right) d \sigma .
\end{aligned}
$$

Then observe the control on $b_{s}$ in (3-132) and the estimate (3-178).

Remark 4.5 (optimality of (4-205)). Corollary 4.4 is the best possible integrability of $e^{\frac{\delta}{b}} / \lambda^{2}$ for constant $\delta$. As a heuristic, assume that $\lambda \sim \sqrt{T-t}$ and $e^{\frac{1}{b}} \sim|\log \lambda| \sim|\log (T-t)|$, motivated by the $\log -\log$ rate H1.3. The integral $\int^{T} \frac{|\log (T-t)|^{\delta}}{T-t} d t$ is only finite for values of $\delta$ sufficiently negative. In our case, the maximum threshold for $\delta$ is given dynamically by (3-178).

Next, we translate hypotheses $\mathrm{H} 2.1-\mathrm{H} 2.3$ into a gain of derivative during particular $N$-dimensional Sobolev embeddings. Consider a smooth cutoff function with support on $\chi^{-1}(\{1\})$ :

$$
\tilde{\chi}(r, z, \theta)= \begin{cases}1 & \text { for }|(r, z)-(1,0)| \geq \frac{3}{4}, \\ 0 & \text { for }|(r, z)-(1,0)| \leq \frac{2}{3} .\end{cases}
$$

Lemma 4.6 (consequences of bootstrap hypotheses). Let $v=\tilde{\chi} u$. Then,

$$
\int\left|\nabla^{N-1} v\right|^{2}|v|^{2} \leq C\left(\tilde{\chi}, \alpha^{*}\right)\|v\|_{H^{N}}^{2},
$$

where $C\left(\tilde{\chi}, \alpha^{*}\right) \rightarrow 0$ as $\alpha^{*} \rightarrow 0$. Furthermore, suppose that

$$
\begin{aligned}
N-1 \geq l_{1} \geq l_{2} \geq l_{3} \geq 0 \quad \text { with } & l_{1}+l_{2}+l_{3}=N, \\
k_{1} \geq k_{2} \geq k_{3} \geq 0 \quad \text { with } & k_{1}+k_{2}+k_{3}=N-2 .
\end{aligned}
$$

Then

$$
\begin{aligned}
\int\left|\nabla^{N} v\right|\left|\nabla^{l_{1}} v\right|\left|\nabla^{l_{2}} v\right|\left|\nabla^{l_{3}} v\right|+\int\left|\nabla^{k_{1}} v\right|\left|\nabla^{k_{2}} v\right|\left|\nabla^{k_{3}} v\right|\left|\nabla^{l_{1}} v\right|\left|\nabla^{l_{2}} v\right|\left|\nabla^{l_{3}} v\right| \\
+\int\left|\nabla^{N-1} v\right|^{2}\left(|\nabla v|^{2}+|v|^{4}\right) \leq C(\tilde{\chi}) \frac{1}{\lambda^{2 N+1}} .
\end{aligned}
$$

Proof. For (4-207), apply the $N$-dimensional Sobolev embeddings $H^{1} \hookrightarrow L^{\frac{2 N}{N-2}}$ and $H^{\frac{N}{2}-1} \hookrightarrow L^{N}$ :

$$
\int\left|\nabla^{N-1} v\right|^{2}|v|^{2} \leq\left\|\nabla^{N-2} v\right\|_{L^{\frac{2 N}{N-2}}}^{2}\|v\|_{L^{N}}^{2} \lesssim\|v\|_{H^{N}}^{2}\|v\|_{H^{\frac{N}{2}-1}}^{2} .
$$

Then recall hypothesis $\mathrm{H} 2.3$. 
We consider in turn the three integrals in (4-208), applying Hölder and $N$-dimensional Sobolev embeddings in each case. For the first,

$$
\int\left|\nabla^{N} v\right|\left|\nabla^{l_{1}} v\right|\left|\nabla^{l_{2}} v\right|\left|\nabla^{l_{3}} v\right| \lesssim\|v\|_{\dot{H}^{N}} \prod_{j=1,2,3}\left\|\nabla^{l_{j}} v\right\|_{L^{\frac{2 N}{l_{j}}}} \lesssim\|v\|_{\dot{H}^{N}} \prod_{j=1,2,3}\|v\|_{H^{\frac{N}{2}}+\frac{l_{j}}{2}+\delta},
$$

where $\frac{1}{2} \gg \delta>0$ is only necessary if $l_{3}=0$. Apply hypotheses $\mathrm{H} 2.1$ and $\mathrm{H} 2.2$, interpolating if $\delta \neq 0$. The resulting bound is of the order $1 / \lambda^{2 N}$.

To deal with the second integral in (4-208), choose $r_{j}=2 N \frac{N-2}{N-2} \frac{1}{k_{j}}$ and $q_{j}=2 N \frac{N}{N+1} \frac{1}{l_{j}}$, so

$$
\sum \frac{1}{r_{j}}=\frac{N-1}{2 N} \text { and } \sum \frac{1}{q_{j}}=\frac{N+1}{2 N} .
$$

Then

$$
\begin{aligned}
\int\left|\nabla^{k_{1}} v\right|\left|\nabla^{k_{2}} v\right|\left|\nabla^{k_{3}} v\right|\left|\nabla^{l_{1}} v\right|\left|\nabla^{l_{2}} v\right|\left|\nabla^{l_{3}} v\right| & \lesssim \prod_{j=1,2,3}\left\|\nabla^{k_{j}} v\right\|_{L^{r_{j}}}\left\|\nabla^{l_{j}} v\right\|_{L^{q_{j}}} \\
& \lesssim \prod_{j=1,2,3}\|v\|_{H^{\frac{N}{2}}+\frac{k_{j}}{2} \frac{N-3}{N-2}+\delta}\|v\|_{H^{\frac{N}{2}+\frac{l_{j}}{2} \frac{N-1}{N}+\delta}}
\end{aligned}
$$

where, again, $\frac{1}{2} \gg \delta>0$ is only necessary if $k_{2}, k_{3}$ or $l_{3}=0$. Apply hypotheses $\mathrm{H} 2.1-\mathrm{H} 2.3$, interpolating where necessary. The resulting bound is of the order $1 / \lambda^{2 N-4}$.

For the third integral, we write

$$
\begin{aligned}
\int\left|\nabla^{N-1} v\right|^{2}\left(|\nabla v|^{2}+|v|^{4}\right) & \lesssim\left\|\nabla^{N-1} v\right\|_{L^{\frac{2 N}{N-2}}}^{2}\left(\|\nabla v\|_{L^{N}}^{2}+\|v\|_{L^{2 N}}^{4}\right) \\
& \lesssim\|v\|_{H^{N}}^{2}\left(\|v\|_{H^{\frac{N}{2}}}^{2}+\|v\|_{H^{\frac{N}{2}-1}}^{4}\right) .
\end{aligned}
$$

Apply hypotheses H2.1 and H2.3. The resulting bound is of the order $1 / \lambda^{2 N}$.

Finally, use hypothesis H1.3 to estimate the neglected factors of $e^{\frac{1}{b}}$ by a single factor of $1 / \lambda$.

Near the singular ring, and in particular on the support of $\nabla \chi$, we do not have the luxury of bootstrap hypotheses. However, under cylindrical symmetry this region is essentially two-dimensional. Indeed, two-dimensional type Sobolev embeddings may be applied to functions supported on this region, as we remark in the next paragraph. Coupled to the geometric decomposition, these embeddings will achieve precisely the weakest usable bounds.

Remark 4.7 (comparison of $H^{v}\left(\mathbb{R}^{2}\right)$ and $H^{v}\left(\mathbb{R}^{N}\right)$ ). Consider $\Omega_{N} \subset\{0<R<r<2 R<\infty\} \subset \mathbb{R}^{N}$, a fixed cylindrical symmetric compact domain away from the origin, as is, in particular, the support of $\nabla \chi$. Let $\Omega_{2} \subset \mathbb{R}^{2}$ denote the obvious projection, and let $f$ denote any cylindrically symmetric function supported on $\Omega_{N}$. For $v \geq 0$ we claim that

$$
\|f\|_{H^{\nu}\left(\mathbb{R}^{2}\right)} \approx_{R, N, v}\|f\|_{H^{\nu}\left(\mathbb{R}^{N}\right)} \quad \text { whenever } f \in H_{0}^{\lceil\nu\rceil}\left(\Omega_{N}\right) .
$$


The canonical linear mapping $T: f\left(x \in \mathbb{R}^{N}\right) \rightarrow f\left((r, z) \in \mathbb{R}^{2}\right)$ is seen, by explicit computation, to be continuous as a map $L_{0}^{2}\left(\Omega_{N}\right) \rightarrow L_{0}^{2}\left(\Omega_{2}\right)$ or $H_{0}^{\lceil\nu\rceil}\left(\Omega_{N}\right) \rightarrow H_{0}^{\lceil\nu\rceil}\left(\Omega_{2}\right)$. Moreover, the mapping is compact (see [Lions 1982]), so the same map between the interpolation spaces $H_{0}^{v}\left(\Omega_{N}\right), H_{0}^{v}\left(\Omega_{2}\right)$ of the interpolation pairs $L^{2}\left(\Omega_{N}\right), H^{\lceil\nu\rceil}\left(\Omega_{N}\right)$ and $L^{2}\left(\Omega_{2}\right), H^{\lceil\nu\rceil}\left(\Omega_{2}\right)$ is also compact [Persson 1964].

Lemma 4.8 (two-dimensional version of Lemma 4.6). Let $v=(1-\tilde{\chi}) u$. There exists $\sigma_{5}>0$ such that

$$
\int\left|\nabla^{N-1} v\right|^{2}|v|^{2} \leq C\left(\tilde{\chi}, \tilde{Q}_{b}\right)\left(\frac{1}{\lambda^{2 N}}+e^{-\frac{\sigma_{5}}{b}}\|u\|_{H^{N}}^{2}\right),
$$

and that

$$
\begin{aligned}
\int\left|\nabla^{N} v\right|\left|\nabla^{l_{1}} v\right|\left|\nabla^{l_{2}} v\right|\left|\nabla^{l_{3}} v\right|+\int\left|\nabla^{k_{1}} v\right|\left|\nabla^{k_{2}} v\right|\left|\nabla^{k_{3}} v\right|\left|\nabla^{l_{1}} v\right|\left|\nabla^{l_{2}} v\right|\left|\nabla^{l_{3}} v\right| \\
+\int\left|\nabla^{N-1} v\right|^{2}\left(|\nabla v|^{2}+|v|^{4}\right) \leq C\left(\tilde{\chi}, \tilde{Q}_{b}\right)\left(\frac{1}{\lambda^{2 N+2}}+\frac{e^{-\frac{\sigma_{5}}{b}}}{\lambda^{2}}\|u\|_{H^{N}}^{2}\right),
\end{aligned}
$$

where $k_{j}$ and $l_{j}$ are as in Lemma 4.6. The value of $\sigma_{5}>0$ is uniform over all $m>0$ sufficiently small. Proof. Due to the concentrated support of $\widetilde{Q}_{b}$ - see (2-55) — we have

$$
(1-\tilde{\chi}) u(r, z, \theta)=\frac{1}{\lambda} \tilde{Q}_{b}\left(\frac{(r, z)-\left(r_{0}, z_{0}\right)}{\lambda}\right) e^{-i \gamma}+(1-\tilde{\chi}) \tilde{u}(r, z),
$$

which we denote by $W+w$. Due to Lemma 2.2, the various norms of $W$ are explicit. For example, $\left\|\nabla^{N} W\right\|_{L^{\infty}} \leq C\left(\widetilde{Q}_{b}\right) / \lambda^{N+1}$, where the constant is uniform over all $b$ sufficiently small. To prove (4-212) and (4-213), we substitute $v=W+w$ and consider two cases: all factors are $W$, or, at least one factor is $w$. The first case is explicit and trivial. In the second case we will extract a factor that is a power of $\|w\|_{H^{1}}$. Assuming $m>0$ is sufficiently small, H1.2 will then yield the factor of $e^{-\sigma_{5} / b}$. Throughout this proof, we preserve the correct multiplicity of $1 / \lambda$ and $\|u\|_{H^{N}}$ by avoiding the Sobolev embedding into $L^{\infty}$.

Make the substitution $v=W+w$. To prove (4-212) we need to show the same bound for,

$$
\int\left|\nabla^{N-1} w\right|\left|\nabla^{N-1} v\right||v|^{2}+\int\left|\nabla^{N-1} v\right|^{2}|v||w|
$$

In the first case, apply the two-dimensional embedding $H^{\frac{1}{2}} \hookrightarrow L^{4}$, and interpolate:

$$
\begin{aligned}
\int\left|\nabla^{N-1} w\right|\left|\nabla^{N-1} v\right||v|^{2} & \leq\left\|\nabla^{N-1} w\right\|_{L^{4}}\left\|\nabla^{N-1} v\right\|_{L^{4}}\|v\|_{L^{4}}^{2} \\
& \lesssim\|w\|_{H^{N-\frac{1}{2}}}\|v\|_{H^{N-\frac{1}{2}}}\|v\|_{H^{\frac{1}{2}}}^{2} \\
& \lesssim\left(\|w\|_{H^{N}}^{1-\frac{1}{2(N-1)}}\|w\|_{H^{1}}^{\frac{1}{2(N-1)}}\right)\|v\|_{H^{N-\frac{1}{2}}}\|v\|_{H^{\frac{1}{2}}}^{2} .
\end{aligned}
$$

Interpolate the norms in $v$ between $\|u\|_{L^{2}}$ and $\|u\|_{H^{N}}$. The factor of $\|w\|_{H^{1}}$ provides a factor of $e^{-\frac{\sigma_{5}}{b}}$ for some $\sigma_{5}>0$, assuming that the constant $m>0$ of hypothesis H2.1 is sufficiently small. For the 
second term of (4-215) follow the same strategy, except use the interpolation $\|w\|_{H^{\frac{1}{2}}} \lesssim\|w\|_{H^{1}}^{\frac{1}{2}}\|w\|_{L^{2}}^{\frac{1}{2}}$. This completes the proof of (4-212).

Now consider the three left-hand side terms of (4-213) in turn. In each case make the substitution $v=W+w$ and assume at least one factor is $w$.

1. We need to show the same bound for

$$
\int\left|\nabla^{N} w\right|\left|\nabla^{l_{a}} W\right|\left|\nabla^{l_{b}} W\right|\left|\nabla^{l_{c}} W\right|+\int\left|\nabla^{N} v\right|\left|\nabla^{l_{a}} v\right|\left|\nabla^{l_{b}} v\right|\left|\nabla^{l_{c}} w\right|,
$$

where $N-1 \geq l_{a}, l_{b}, l_{c} \geq 0$, with $l_{a}+l_{b}+l_{c}=N$, is some permutation of $l_{1}, l_{2}, l_{3}$. Integrate the first term of (4-217) by parts, use Hölder and interpolate:

$$
\left\|\nabla^{N-1} w\right\|_{L^{2}}\left\|\nabla^{l_{a}} W \nabla^{l_{b}} W \nabla^{l_{c}} W\right\|_{H^{1}} \lesssim\|w\|_{H^{N}}^{1-\frac{1}{N-1}}\|w\|_{H^{1}}^{\frac{1}{N-1}}\left\|\nabla^{l_{a}} W \nabla^{l_{b}} W \nabla^{l_{c}} W\right\|_{H^{1}} .
$$

The norms of $W$ have explicit scaling-consistent bounds of the order $(1 / \lambda)^{\left(2+l_{a}+l_{b}+l_{c}\right)}$. Again, $\|w\|_{H^{1}}$ provides a factor of $e^{-\frac{\sigma_{5}}{b}}$ for some $\sigma_{5}>0$ and the resulting bound is of the order $1 / \lambda^{2 N+1}$.

The remaining term of (4-217) is more difficult. Choose $q_{a}, q_{b}, q_{c}>0$ such that

$$
\sum_{j=a, b, c} \frac{1}{q_{j}}=\frac{1}{2} \quad \text { with } \frac{1}{q_{j}}<\frac{l_{j}}{2} \text { if } l_{j} \neq 0 \quad \text { and } \quad \frac{1}{q_{j}}<\sigma_{4} \text { if } l_{j}=0,
$$

where $0<\sigma_{4} \ll 1$ is an arbitrary universal constant. Apply Hölder and two-dimensional Sobolev embeddings

$$
\begin{aligned}
& \int\left|\nabla^{N} v\right|\left|\nabla^{l_{a}} v\right|\left|\nabla^{l_{b}} v\right|\left|\nabla^{l_{c}} w\right| \leq\|v\|_{H^{N}}\left\|\nabla^{l_{a}} v\right\|_{L^{q_{a}}}\left\|\nabla^{l_{b}} v\right\|_{L^{q_{b}}}\left\|\nabla^{l_{c}} w\right\|_{L^{q_{c}}}
\end{aligned}
$$

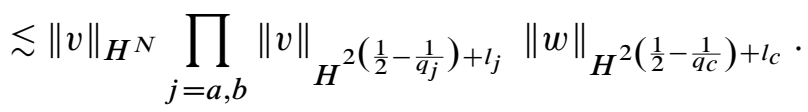

Due to the choice in (4-218), the final three norms of (4-219) may be interpolated strictly between $H^{N}$ and $H^{1}$, or strictly between $H^{1}$ and $L^{2}$, if $l_{j}=0$. We are guaranteed a factor in $\|w\|_{H^{1}}$,

$$
(4-219) \lesssim \begin{cases}\|u\|_{H^{N}}^{2}\|u\|_{H^{1}}^{2-C\left(l_{c}\right)}\|w\|_{H^{1}}^{C\left(l_{c}\right)} & \text { if all the } l_{j} \text { are nonzero, } \\ \|u\|_{H^{N}}^{2+C\left(\sigma_{4}\right)}\|u\|_{H^{1}}^{2-N C\left(\sigma_{4}\right)-C\left(l_{c}\right)}\|w\|_{H^{1}}^{C\left(l_{c}\right)} & \text { if some } l_{j} \text { is zero. }\end{cases}
$$

For $m>0$ sufficiently small (relative to $\sigma_{4}$ ), there is a spare factor of $e^{-\frac{\sigma_{5}}{b}}$, for some $\sigma_{5}>0$. This proves the bound for the first term on the left in (4-213).

2. Choose $r_{1}, r_{2}, r_{3}>0$ and $q_{1}, q_{2}, q_{3}>0$ according to the rules of (4-218). Then,

$$
\int\left|\nabla^{k_{1}} v\right|\left|\nabla^{k_{2}} v\right|\left|\nabla^{k_{3}} v\right|\left|\nabla^{l_{1}} v\right|\left|\nabla^{l_{2}} v\right|\left|\nabla^{l_{3}} v\right| \lesssim \prod_{j=1,2,3}\left\|\nabla^{k_{j}} v\right\|_{L^{r_{j}}}\left\|\nabla^{l_{j}} v\right\|_{L^{q_{j}}}
$$

Recall that at least one factor of $v$ in (4-221) is in fact $w$. Continue with two-dimensional Sobolev embeddings and interpolation as we did at Equation (4-219). This proves the bound for the second term on the left in (4-213). 
3. Apply Hölder and two-dimensional Sobolev to get

$$
\begin{aligned}
\int\left|\nabla^{N-1} w\right|\left|\nabla^{N-1} v\right||\nabla v|^{2} & \leq\left\|\nabla^{N-1} w\right\|_{L^{4}}\left\|\nabla^{N-1} v\right\|_{L^{4}}\|\nabla v\|_{L^{4}}^{2} \\
& \lesssim\|w\|_{H^{N-\frac{1}{2}}}\|v\|_{H^{N-\frac{1}{2}}\|v\|_{H^{\frac{3}{2}}}^{2}} \\
\int\left|\nabla^{N-1} w\right|\left|\nabla^{N-1} v\right||v|^{4} & \leq\left\|\nabla^{N-1} w\right\|_{L^{4}}\left\|\nabla^{N-1} v\right\|_{L^{4}}\|v\|_{L^{8}}^{4} \\
& \lesssim\|w\|_{H^{N-\frac{1}{2}}}\|v\|_{H^{N-\frac{1}{2}}}\|v\|_{H^{\frac{3}{4}}}^{4}
\end{aligned}
$$

The bound for the third term on the left-hand side of (4-213) follows from interpolation.

Lemma 4.9 $\left(H^{N}\right.$ energy identity). Denote the $N$-th order energy by

$$
E_{N}(u)=\int\left|\nabla^{N} u\right|^{2}-\left(2 \int\left|\nabla^{N-1} u\right|^{2}|u|^{2}+\operatorname{Re} \int\left(\nabla^{N-1} \bar{u}\right)^{2} u^{2}\right) .
$$

Then

$$
\begin{array}{r}
\frac{1}{C}\left|\frac{d}{d t} E_{N}(u)\right| \leq \int\left|\nabla^{N} u\right|\left|\nabla^{l_{1}} u\right|\left|\nabla^{l_{2}} u\right|\left|\nabla^{l_{3}} u\right|+\int\left|\nabla^{k_{1}} u\right|\left|\nabla^{k_{2}} u\right|\left|\nabla^{k_{3}} u\right|\left|\nabla^{l_{1}} u\right|\left|\nabla^{l_{2}} u\right|\left|\nabla^{l_{3}} u\right| \\
+\int\left|\nabla^{N-1} u\right|^{2}\left(|\nabla u|^{2}+|u|^{4}\right)
\end{array}
$$

where the right-hand side is implicitly summed over $N-1 \geq l_{1} \geq l_{2} \geq l_{3} \geq 0$ with $l_{1}+l_{2}+l_{3}=N$ and $k_{1} \geq k_{2} \geq k_{3} \geq 0$ with $k_{1}+k_{2}+k_{3}=N-2$.

Proof. We refer to the right-hand side of (4-225) as error terms of type I, II, and III respectively. By direct calculation,

$$
\begin{aligned}
\frac{1}{2} \frac{d}{d t}\left(\int\left|\nabla^{N} u\right|^{2}\right) & =-\operatorname{Im} \int \nabla^{N}\left(\Delta u+u|u|^{2}\right) \nabla^{N} \bar{u} \\
& =-2 \operatorname{Im} \int \nabla\left(\nabla^{N-1} u|u|^{2}\right) \nabla^{N} \bar{u}-\operatorname{Im} \int \nabla\left(\nabla^{N-1} \bar{u} u^{2}\right) \nabla^{N} \bar{u} \\
& + \text { terms of the form } \int \nabla^{N-2}(\nabla u \nabla u u) \nabla^{N} \bar{u} .
\end{aligned}
$$

The final terms here are errors of type I. Regarding the first term on the right in (4-226),

$$
\begin{aligned}
-2 \operatorname{Im} \int \nabla\left(\nabla^{N-1} u|u|^{2}\right) \nabla^{N} \bar{u} & =2 \operatorname{Im} \int \nabla^{N-1} u|u|^{2} \nabla^{N-1} \Delta \bar{u} \\
& =\int \frac{d}{d t}\left(\left|\nabla^{N-1} u\right|^{2}\right)|u|^{2}-2 \operatorname{Im} \int \nabla^{N-1} u|u|^{2} \nabla^{N-1}\left(\bar{u}|u|^{2}\right) .
\end{aligned}
$$

Recognize the last term of (4-227) as error of type II and III. Regarding the other term,

$$
\int \frac{d}{d t}\left(\left|\nabla^{N-1} u\right|^{2}\right)|u|^{2}=\frac{d}{d t}\left(\int\left|\nabla^{N-1} u\right|^{2}|u|^{2}\right)+2 \operatorname{Im} \int\left|\nabla^{N-1} u\right|^{2}\left(\Delta u+u|u|^{2}\right) \bar{u} .
$$


After integration by parts, we recognize the final term of (4-228) as being of type I and III. We have shown that

$$
-2 \operatorname{Im} \int \nabla\left(\nabla^{N-1} u|u|^{2}\right) \nabla^{N} \bar{u}=\frac{1}{2} \frac{d}{d t}\left(\int\left|\nabla^{N-1} u\right|^{2}|u|^{2}\right),
$$

up to error terms. It is virtually the same calculation to show that,

$$
-\operatorname{Im} \int \nabla\left(\nabla^{N-1} \bar{u} u^{2}\right) \nabla^{N} \bar{u}=\frac{1}{2} \frac{d}{d t}\left(\operatorname{Re} \int\left(\nabla^{N-1} \bar{u}\right)^{2} u^{2}\right),
$$

also up to error terms of type I, II, and III. This completes the proof of (4-225).

Now we simply combine the previous three Lemmas. Equations (4-207) and (4-212) prove that $E_{N} \approx$ $\|u\|_{H^{N}}$. Equations (4-208) and (4-213) control $d E_{N} / d t$. Integrate the bound on $d E_{N} / d t E_{N}$ using Lemma 4.3, with $\sigma_{2}<\min \left(\sigma_{5}, 2 m\right)$. Choose $m^{\prime}>0$ to be any value, $m-\sigma_{5} / 2<m^{\prime}<m$. Assuming $\alpha^{*}$ is sufficiently small (depending on the choice of $m^{\prime}$ ), we have proved statement I2.1:

Lemma 4.10 (controlled growth of $H^{N}$ ). For all $t \in\left[0, T_{\text {hyp }}\right.$ ),

$$
\|u(t)\|_{H^{N}\left(\mathbb{R}^{N}\right)}<\frac{e^{\frac{m^{\prime}}{b(t)}}}{\lambda^{N}(t)} .
$$

Behavior away from both infinity and the singularity. In this section we concentrate on the interface between the singular set and the truly $N$-dimensional region that contains the origin. On this interface, away from $r=0$, the dynamics remains essentially two-dimensional and $L^{2}$-critical

Lemma 4.11 (Two-dimensional endpoint Sobolev control away from the singularity). If $\sigma_{6}>0$ and $\varphi$ is a smooth cutoff function compactly supported away from both the singular set and the origin,

$$
\|\varphi u(t)\|_{L^{\infty}\left(\mathbb{R}^{N}\right)} \leq C\left(\sigma_{6}, \varphi\right) e^{+\frac{\sigma_{5}}{b(t)}}\left(\|\tilde{u}(t)\|_{H^{1}\left(\mathbb{R}^{N}\right)}+\frac{\Gamma_{b(t)}^{\frac{1}{2}}}{\lambda(t)}\right) .
$$

(This is a two-dimensional type of estimate due to the support of $\varphi$.)

The key feature of Lemma 4.11 is that it lets us avoid the Sobolev embedding $H^{1+\epsilon}\left(\mathbb{R}^{2}\right) \hookrightarrow L^{\infty}\left(\mathbb{R}^{2}\right)$. At the order of the blowup parameter $\lambda$, Equation (4-230) is consistent with scaling. The analogue in the case of radial symmetry, given in [Raphaël 2006; Raphaël and Szeftel 2009], is Strauss's radial embedding.

Proof of Lemma 4.11. We adapt an argument of Brezis and Gallouët. Our estimate is for a fixed time $t \in\left[0, T_{\text {hyp }}\right)$. Choose

$$
R=\|\tilde{u}(t)\|_{H^{1}}+\frac{\Gamma_{b(t)}^{\frac{1}{2}}}{\lambda(t)} \gg 0 .
$$

Consider $v=\varphi u$ as a compactly supported function of two variables, and partition phase space as follows:

$$
|v| \leq\|\hat{v}\|_{L^{1}\left(\mathbb{R}^{2}\right)}=\int_{|\xi| \leq R}|\hat{v}(\xi)| d \xi+\int_{|\xi|>R}|\hat{v}(\xi)| d \xi
$$


Let $\langle\xi\rangle$ denote $\sqrt{1+|\xi|^{2}}$ and rewrite the low frequencies:

$$
\int_{|\xi| \leq R}|\hat{v}| d \xi=\int_{|\xi| \leq R}\left(\langle\xi\rangle^{\frac{1}{2}}|\hat{v}|^{\frac{1}{2}}\right)\left(|\hat{v}|^{\frac{1}{2}}\right)\left(\langle\xi\rangle^{-\frac{1}{2}}\right) d \xi \leq\|v\|_{H^{1}}^{\frac{1}{2}}\|v\|_{L^{2}}^{\frac{1}{2}}\left(\int_{|\xi| \leq R} \frac{1}{\langle\xi\rangle} d \xi\right)^{\frac{1}{2}},
$$

Estimate the final integral of (4-231) as $\int_{|\xi| \leq R} \frac{1}{\langle\xi\rangle} d \xi \leq \int_{0}^{R} \frac{1}{\rho} \rho d \rho=R$. Apply a similar argument for high frequencies, with parameter $v\left(\sigma_{6}, m\right)>1$ to be determined:

$$
\begin{aligned}
\int_{|\xi|>R}|\hat{v}| d \xi & =\int_{|\xi|>R}\left(\langle\xi\rangle^{v}|\hat{v}|\right) \frac{1}{\langle\xi\rangle^{v}} d \xi \lesssim\|v\|_{H^{v}}\left(\int_{R}^{+\infty} \frac{1}{\langle\rho\rangle^{2 v}} \rho d \rho\right)^{\frac{1}{2}} \\
& \leq \frac{1}{2(v-1)}\|v\|_{H^{v}} \frac{1}{R^{v-1}} \lesssim \frac{1}{2(v-1)}\left(\|v\|_{H^{1}}^{2-v} R^{\nu-1}\right)\left(\frac{\|v\|_{H^{2}}^{\nu-1}}{R^{2(v-1)}}\right) .
\end{aligned}
$$

Due to hypothesis H2.1 and the $\Gamma_{b}$-estimate (3-103), the final term of (4-232) is bounded by $e^{+\frac{\sigma_{6}}{b(t)}}$ for any choice of $v>1$ sufficiently small.

Definition 4.12 (cutoffs to cover Supp $\nabla \chi$ ). Fix $N+4$ smooth cylindrically symmetric cutoff functions $\psi^{(0)}, \psi^{\left(\frac{1}{2}\right)}, \psi^{(1)}, \varphi^{\left(N-\frac{1}{2}\right)}, \varphi^{(N-1)}, \ldots, \varphi^{\left(\frac{N}{2}\right)}, \varphi^{\left(\frac{N}{2}-\frac{1}{2}\right)}$ with the following properties:

1. They cover the support of $\nabla \chi$ : Each function is 1 on $\left\{\frac{1}{3}<|(r, z)-(1,0)|<\frac{2}{3}\right\}$.

2. The tails do not overlap: The support of each cutoff is contained where the previous cutoff is 1 .

3. They are supported away from both the singularity and the origin: The largest support, that of $\psi^{(0)}$, is contained in $\left\{\frac{1}{7}<|(r, z)-(1,0)|<\frac{6}{7}\right\}$.

Lemma 4.13 (annular $H^{\frac{1}{2}}$ control: the crucial first step). For all $t \in\left[0, T_{\text {hyp }}\right.$ ),

$$
\left\|\psi^{\left(\frac{1}{2}\right)} u\right\|_{H^{\frac{1}{2}}} \lesssim \frac{1}{\lambda C\left(\alpha^{*}\right)(t)}
$$

where $C\left(\alpha^{*}\right) \rightarrow 0$ as $\alpha^{*} \rightarrow 0$.

This is the first proof that any behavior better than scaling extends beyond the support of hypotheses $\mathrm{H} 2.2$ and $\mathrm{H} 2.3$.

Remark 4.14 (analogue in [Raphaël 2006; Raphaël and Szeftel 2009]). In radial cases, one proves Lemma 4.13 for $H^{v}, v<\frac{1}{2}$. The subsequent $H^{\frac{1}{2}}$ bound - see, for example, [Raphaël 2006, Lemma 10] — should be seen as comparable to the forthcoming Lemma 4.17 .

Proof of Lemma 4.13. By direct calculation,

$$
\frac{1}{2} \frac{d}{d t}\left\|\psi^{\left(\frac{1}{2}\right)} u\right\|_{\dot{H}^{\frac{1}{2}}}^{2}=\operatorname{Im}\left(\int D^{\frac{1}{2}}\left(u \Delta \psi^{\left(\frac{1}{2}\right)}+2 \nabla \psi^{\left(\frac{1}{2}\right)} \cdot \nabla u-\psi^{\left(\frac{1}{2}\right)} u|u|^{2}\right) D^{\frac{1}{2}}\left(\psi^{\left(\frac{1}{2}\right)} \bar{u}\right)\right) .
$$

Estimate the first and second terms on the right in (4-234):

$$
\begin{aligned}
\left\|D^{\frac{1}{2}}\left(u \Delta \psi^{\left(\frac{1}{2}\right)}\right)\right\|_{L^{2}}\left\|\psi^{\left(\frac{1}{2}\right)} u\right\|_{H^{\frac{1}{2}}} & \leq C\left(\psi^{\left(\frac{1}{2}\right)}\right)\left\|\psi^{(0)} u\right\|_{H^{\frac{1}{2}}}\left\|\psi^{\left(\frac{1}{2}\right)} u\right\|_{H^{\frac{1}{2}}}, \\
\left\|\nabla \psi^{\left(\frac{1}{2}\right)} \cdot \nabla u\right\|_{L^{2}}\left\|\psi^{\left(\frac{1}{2}\right)} u\right\|_{H^{1}} & \leq C\left(\psi^{\left(\frac{1}{2}\right)}\right)\left\|\psi^{(0)} u\right\|_{H^{1}}^{2}
\end{aligned}
$$


The nonlinear term of (4-234) does not enjoy any real-valued cancellations, as the operator $D$ does not have an exact Leibniz property. Apply standard commutation estimates:

$$
\begin{aligned}
\left\|D^{\frac{1}{2}}\left(\psi^{\left(\frac{1}{2}\right)} u|u|^{2}\right)\right\|_{L^{2}} & \lesssim\left\|\psi^{\left(\frac{1}{2}\right)} u\right\|_{H^{\frac{1}{2}}}\left\|\psi^{(0)} u\right\|_{L^{\infty}\left(\mathbb{R}^{2}\right)}^{2}+\left\|\psi^{\left(\frac{1}{2}\right)} u\right\|_{L^{4}}\left\|\psi^{(0)} u\right\|_{W^{\frac{1}{2}, 4}}\left\|\psi^{(0)} u\right\|_{L^{\infty}\left(\mathbb{R}^{2}\right)} \\
& \lesssim\left\|\psi^{\left(\frac{1}{2}\right)} u\right\|_{H^{\frac{1}{2}}}\left(\left\|\psi^{(0)} u\right\|_{L^{\infty}\left(\mathbb{R}^{2}\right)}^{2}+\left\|\psi^{(0)} u\right\|_{H^{1}}\left\|\psi^{(0)} u\right\|_{L^{\infty}\left(\mathbb{R}^{2}\right)}\right)
\end{aligned}
$$

From support away from the singularity, $\psi^{(0)} u=\psi^{(0)} \tilde{u}$, and we may apply the endpoint estimate of Lemma 4.11. Denote $\left\|\psi^{\left(\frac{1}{2}\right)} u(t)\right\|_{\dot{H}^{\frac{1}{2}}}$ by $f$. We have the simple ODE $\frac{1}{2} \frac{d}{d t}\left(f^{2}\right) \leq C\left(\psi^{\left(\frac{1}{2}\right)}\right)\left(f\|\tilde{u}(t)\|_{H^{1}}^{\frac{1}{2}}\|\tilde{u}(t)\|_{L^{2}}^{\frac{1}{2}}+\|\tilde{u}(t)\|_{H^{1}}^{2}\right)+C\left(\sigma_{6}, \psi^{(0)}\right) f^{2} e^{+\frac{\sigma_{6}}{b(t)}}\left(\|\tilde{u}(t)\|_{H^{1}}^{2}+\frac{\Gamma_{b(t)}}{\lambda^{2}(t)}\right)$.

The final term is dominant. After integration, by Corollary 4.4,

$$
\left\|\psi^{\left(\frac{1}{2}\right)} u(t)\right\|_{H^{\frac{1}{2}}} \lesssim e^{\left(C\left(\alpha^{*}\right) C\left(\sigma_{6}, \psi^{(0)}\right) e^{+\frac{\sigma_{6}}{b(t)}}\right)} .
$$

To complete the proof, choose $\sigma_{6}=\frac{\pi}{10}$ and recall the $\log -\log$ rate H1.3.

Remark 4.15 (justification for Lemma 4.11). The open nature of hypothesis H1.3 is an essential feature of any modulation argument. It is for this reason that we must be free to choose $\sigma_{6}$. The standard Brezis-Gallouët estimate, $\|v\|_{L^{\infty}\left(\mathbb{R}^{2}\right)} \lesssim\|v\|_{H^{1}} \sqrt{\log \left(\|v\|_{\left.H^{2}\right)}\right.}$, would not suffice to prove Lemma 4.13.

We now reformulate the calculation of (4-234) for repeated application.

Lemma 4.16 (standard Gronwall argument). Let $\psi^{A}$ be supported where $\psi^{B} \equiv 1$, let I be any subinterval of $\left[0, T_{\mathrm{hyp}}\right)$, and let $v \geq 0$. Then

$$
\left\|\psi^{A} u\right\|_{L_{I}^{\infty} H^{\nu}} \leq C\left(\psi^{A}\right)\left(\left\|\psi^{B} u_{0}\right\|_{H^{v}}+|I|+\left\|\psi^{B} u\right\|_{L_{I}^{2} H^{v+\frac{1}{2}}}+\left\|\psi^{A} u|u|^{2}\right\|_{L_{I}^{1} H^{v}}\right) .
$$

Lemma 4.17 (annular $H^{1}$ control: propagation of Lemma 4.13). There exists $\sigma_{7}>0$, universal for all $m>0$ sufficiently small, such that for, all $t \in\left[0, T_{\mathrm{hyp}}\right)$,

$$
\left\|\psi^{(1)} u(t)\right\|_{H^{1}}<C\left(\alpha^{*}\right) \frac{e^{-\frac{\sigma_{7}}{b(t)}}}{\lambda^{\frac{1}{2}}(t)},
$$

where $C\left(\alpha^{*}\right) \rightarrow 0$ as $\alpha^{*} \rightarrow 0$.

Proof. Apply (4-237) for $v=1, I=\left[0, t<T_{\text {hyp }}\right], \psi^{A}=\psi^{(1)}$, and $\psi^{B}=\psi^{\left(\frac{1}{2}\right)}$. Note that $\psi^{(1)} u=\psi^{(1)} \tilde{u}$. Through interpolation and hypotheses $\mathrm{H} 1.2$ and $\mathrm{H} 2.1$,

$$
\left\|\psi^{(1)} u\right\|_{L_{I}^{2} H^{1+\frac{1}{2}}} \lesssim\left(\int\|\tilde{u}\|_{H^{1}}^{2-\frac{1}{N-1}}\|\tilde{u}\|_{H^{N}}^{\frac{1}{N-1}}\right)^{\frac{1}{2}} \lesssim\left(\int e^{-\left(\frac{1}{4}-\frac{m}{N-1}\right) \frac{1}{b}} \frac{1}{\lambda^{N}}\right)^{\frac{1}{2}} .
$$

Assuming $m>0$ is sufficiently small, apply integrability Lemma 4.3 for $\sigma_{2}>0$, also sufficiently small. Regarding the final term of (4-237), apply Hölder, two-dimensional Sobolev embedding, and interpolate: 


$$
\begin{aligned}
& \left\|\psi^{(1)} u|u|^{2}\right\|_{H^{1}} \lesssim\left\|\nabla\left(\psi^{\left(\frac{1}{2}\right)} u\right)\left(\psi^{\left(\frac{1}{2}\right)} u\right)^{2}\right\|_{L^{2}} \lesssim\left\|\nabla\left(\psi^{\left(\frac{1}{2}\right)} u\right)\right\|_{L^{4}}\left\|\psi^{\left(\frac{1}{2}\right)} u\right\|_{L^{8}}^{2} \lesssim\left\|\psi^{\left(\frac{1}{2}\right)} u\right\|_{H^{\frac{3}{2}}}\left\|\psi^{\left(\frac{1}{2}\right)} u\right\|_{H^{\frac{3}{4}}}^{2} \\
& \lesssim\|u\|_{H^{N}}^{\frac{3}{2} \frac{1}{N-\frac{1}{2}}}\left\|\psi\left(\frac{1}{2}\right) u\right\|_{H^{\frac{1}{2}}}^{3-\frac{3}{2} \frac{1}{N-\frac{1}{2}}} \lesssim \frac{1}{\lambda^{\frac{3}{2} \frac{N}{N-\frac{1}{2}}+C\left(\alpha^{*}\right)}(t)},
\end{aligned}
$$

where the final inequality is due to hypothesis H2.1 and Lemma 4.13. The final exponent is less than 2 for $\alpha^{*}>0$ sufficiently small and $N \geq 3$. Apply Lemma 4.1 .

Remark 4.18 (scheme for the remainder of Section 4). The proof of Lemma 4.17 may be repeated, with a shrunken cutoff and $H^{\frac{3}{2}}$ in place of $H^{1}$. However, due to the new version of Equation (4-239), iteration to higher norms will not yield more than the same $\frac{1}{2}$-derivative improvement over scaling.

Instead, we switch direction. Starting with I2.1, at each stage the previous iterate will give progressively better control on the equivalent of (4-239). Lemma 4.17 will be used to help control the equivalent of Equation (4-240).

Lemma 4.19 (Moser-type product estimate). Let $v \in H^{v+\frac{1}{2}}\left(\mathbb{R}^{d}\right)$ for some $v \geq \frac{d}{2}-\frac{1}{2}$, not necessarily an integer. Then,

$$
\left\|v^{3}\right\|_{H^{v}} \lesssim\|v\|_{H^{v+\frac{1}{2}}}\|v\|_{H^{\frac{d}{2}}}^{2}
$$

Lemma 4.20 (I2.2 and I2.3 on the support of $\nabla \chi$ ). For any $t \in\left[0, T_{\text {hyp }}\right.$ ) and any half-integer $\frac{1}{2} \leq \kappa<\frac{N}{2}$, we have

$$
\begin{gathered}
\left\|\varphi^{(N-\kappa)} u\right\|_{H^{N-\kappa}}<C\left(\alpha^{*}\right) \frac{e^{(1+\kappa) \frac{m^{\prime}}{b(t)}}}{\lambda^{N-2 \kappa}}, \\
\left\|\varphi^{\left(\frac{N}{2}\right)} u(t)\right\|_{H^{\frac{N}{2}}}<C\left(\alpha^{*}\right) e^{+\frac{2 m^{\prime}+\pi}{b(t)}} \\
\left\|\varphi^{\left(\frac{N}{2}-\frac{1}{2}\right)} u\right\|_{H^{\frac{N}{2}-\frac{1}{2}}}<C\left(\alpha^{*}\right)\left(\alpha^{*}\right)^{\frac{1}{5}}
\end{gathered}
$$

where in each case $C\left(\alpha^{*}\right) \rightarrow 0$ as $\alpha^{*} \rightarrow 0$.

Proof. We prove (4-242) by induction in $\kappa$. The base case $\kappa=0$ is Lemma 4.10. Hypothesize that (4-242) holds for $\kappa-\frac{1}{2}$, some $\kappa \geq \frac{1}{2}$. Set $v=N-\kappa$ and apply the standard Gronwall argument for $I=\left[0, t<T_{\text {hyp }}\right], \psi^{A}=\phi^{(v)}$ and $\psi^{B}=\phi^{\left(v+\frac{1}{2}\right)}$,

$$
\left\|\varphi^{(v)} u\right\|_{H^{v}} \lesssim\left\|\chi_{0} u_{0}\right\|_{H^{\nu}}+\left\|\varphi^{\left(v+\frac{1}{2}\right)} u\right\|_{L_{t}^{2} H^{\nu+\frac{1}{2}}}+\left\|\varphi^{(v)} u|u|^{2}\right\|_{L_{t}^{1} H^{v}}
$$

Apply our induction hypothesis to the second term on the right in (4-245):

$$
\left\|\varphi^{\left(\nu+\frac{1}{2}\right)} u\right\|_{L_{t}^{2} H^{\nu+\frac{1}{2}}} \lesssim\left(\int_{I}\left(\frac{e^{\left(1+\left(\kappa-\frac{1}{2}\right)\right) \frac{m^{\prime}}{b(\tau)}}}{\lambda^{N-2\left(\kappa-\frac{1}{2}\right)}(\tau)}\right)^{2} d \tau\right)^{\frac{1}{2}} \lesssim \frac{e^{(1+\kappa) \frac{m^{\prime}}{b(t)}}}{\lambda^{N-2 \kappa}(t)}\left(e^{\frac{\sigma_{2}-m^{\prime}}{b(t)}}\right)^{\frac{1}{2}} .
$$

where, since $\kappa<\frac{N}{2}$, we applied Lemma 4.3 for some $\sigma_{2}<m^{\prime}$. Examine the final term of (4-245). Note that, $\varphi^{(v)} u=\varphi^{(v)}\left(\varphi^{\left(v+\frac{1}{2}\right)} u\right)$. Recall Remark 4.7, apply Lemma 4.19 in the two-dimensional case, and 
inject both the induction hypothesis and the $H^{1}$ control of Lemma 4.17,

$$
\begin{aligned}
\left\|\varphi^{(v)} u|u|^{2}\right\|_{H^{v}} & \lesssim\left\|\varphi^{\left(v+\frac{1}{2}\right)} u\right\|_{H^{v+\frac{1}{2}}}\left\|\varphi^{\left(v+\frac{1}{2}\right)} u\right\|_{H^{1}}^{2} \\
& \lesssim \frac{e^{+\frac{\left(1+\left(\kappa-\frac{1}{2}\right)\right) m^{\prime}}{b}}}{\lambda^{N-2\left(\kappa-\frac{1}{2}\right)}} \frac{e^{-\frac{2 \sigma_{7}}{b}}}{\lambda}=\frac{e^{\frac{(\mathrm{neg})}{b}}}{\lambda^{2}} \frac{1}{\lambda^{N-2 \kappa}},
\end{aligned}
$$

where we made the assumption that $m>0$ is sufficiently small relative to $\sigma_{7}$. Finally, apply Lemma 4.3 for some $\sigma_{2}$ less than the negative exponent. This completes the proof of (4-242).

To prove (4-243) let $\kappa=\frac{N}{2}$. We proceed exactly as above, using (4-242) in place of the induction hypothesis, and applying Corollary 4.4 in place of Lemma 4.3.

To prove (4-244), let $\kappa=\frac{N}{2}+\frac{1}{2}$. We proceed exactly as above using (4-243) in place of the induction hypothesis, and applying Lemma 4.1 in place of Lemma 4.3.

Improved behavior at infinity. With Lemma 4.20 covering the support of $\nabla \chi$, we prove the corresponding result for $\chi$ by similar methods. Note the argument is now in three dimensions.

Proof of I2.2 and I2.3. We revisit the proof of the standard Gronwall argument. Let $I=\left[0, t<T_{\text {hyp }}\right]$, $v \geq 0$, and set $v=\chi u$. With (1-1), we get

$$
i v_{t}+\Delta v+v|v|^{2}=u \Delta \chi+2 \nabla \chi \cdot \nabla u+\left(\chi^{2}-1\right) \chi u|u|^{2} .
$$

Note that the terms on the right-hand side of (4-248) are localized to the support of $\nabla \chi$, a region of two-dimensional character where $\varphi^{\left(\frac{N}{2}-\frac{1}{2}\right)} \equiv 1$. By direct calculation,

$$
\begin{aligned}
\frac{1}{2}\|\chi u\|_{L_{I}^{\infty} H^{\nu}} \leq \| & \chi u_{0}\left\|_{H^{\nu}}+\right\| \chi u|\chi u|^{2} \|_{L_{I}^{1} H^{\nu}} \\
& +C(\chi)\left(\left\|\varphi^{(1)} u_{0}\right\|_{H^{\nu}}+|I|+\left\|\varphi^{(1)} u\right\|_{L_{I}^{2} H^{\nu+\frac{1}{2}}}+\left\|\varphi^{(1)} u\left|\varphi^{(1)} u\right|^{2}\right\|_{L_{I}^{1} H^{\nu}}\right) .
\end{aligned}
$$

Consider $\nu=N-\kappa$ for some $\kappa \in\left[\frac{1}{2}, \frac{N}{2}+\frac{1}{2}\right]$. Due to Definition 4.12, all the conclusions of Lemma 4.20 apply to $\varphi^{\left(\frac{N}{2}-\frac{1}{2}\right)} u$, which we use in place of an induction hypothesis to control the second line of (4-249), exactly as we did Equation (4-245). These terms will give the largest contribution.

Finally, examine the term nonlinear in $\chi u$. Apply the Moser-type estimate of Lemma 4.19, interpolate, and inject hypotheses $\mathrm{H} 2.2$,

$$
\begin{aligned}
& \left\|\chi u|\chi u|^{2}\right\|_{L_{I}^{1} H^{N-\kappa}} \lesssim\|\| \chi u\left\|_{H^{N-\left(\kappa-\frac{1}{2}\right)}}\right\| \chi u\left\|_{H^{\frac{N}{2}}}^{2}\right\|_{L_{I}^{1}} \\
& \lesssim \begin{cases}\int_{I} \frac{e^{\left(1+\left(\kappa-\frac{1}{2}\right)\right) \frac{m}{b(\tau)}} e^{2\left(\frac{2 m+\pi}{b(\tau)}\right)} d \tau}{\lambda^{N-2\left(\kappa-\frac{1}{2}\right)}(\tau)} & \text { for } \kappa \leq \frac{N}{2}, \\
\int_{I} e^{3\left(\frac{2 m+\pi}{b(\tau)}\right)} d \tau & \text { for } \kappa=\frac{N}{2}+\frac{1}{2} .\end{cases}
\end{aligned}
$$

Apply Lemma 4.1 for $\kappa \geq \frac{N}{2}$, Corollary 4.4 for $\kappa=\frac{N}{2}-\frac{1}{2}$, and Lemma 4.3 for $\frac{N}{2}-1 \geq \kappa \geq \frac{1}{2}$. Note that the result of Equation (4-250) is an entire order better than necessary.

This completes all the deferred proofs necessary to establish Proposition 2.8. 


\section{Proof of Theorem 1.6}

Proof of norm growth (1-12), (1-13). From Proposition 2.8 we have that $T_{\text {hyp }}=T_{\max }$, and from (3-143) we have blowup in finite time. By the failure of local wellposedness we have that $\lambda(t) \rightarrow 0$ as $t \rightarrow T_{\max }$. Recall the approximate dynamics of $\lambda$, Equation (3-140), which with the control on $b$ implies in particular that $\left|\lambda_{s} / \lambda\right|<1$ on $\left[s_{0}, s_{\max }\right)$, which easily integrates to

$$
|\log \lambda(s)| \lesssim 1+s
$$

Therefore $s_{\max }=+\infty$. By direct calculation and a change of variable,

$$
-\partial_{t}\left(\lambda^{2} \log |\log \lambda|\right)=-\frac{\lambda_{s}}{\lambda} \log |\log \lambda|\left(2+\frac{1}{|\log \lambda| \log |\log \lambda|}\right) .
$$

The approximate dynamics (3-140) gives

$$
\frac{b}{2} \leq-\frac{\lambda_{s}}{\lambda} \leq 2 b
$$

so with the $\log -\log$ rate $\mathrm{H} 1.3$ we have proven that, for some universal constant $C>0$ and all $t \in\left[0, T_{\max }\right)$,

$$
\frac{1}{C} \leq-\partial_{t}\left(\lambda^{2} \log |\log \lambda|\right) \leq C .
$$

For all $t \in\left[0, T_{\max }\right)$, integrate Equation (5-252). Since $\lambda$ is very small we can estimate

$$
\frac{1}{C}\left(\frac{T_{\max }-t}{\log \left|\log \left(T_{\max }-t\right)\right|}\right)^{\frac{1}{2}} \leq \lambda(t) \leq C\left(\frac{T_{\max }-t}{\log \left|\log \left(T_{\max }-t\right)\right|}\right)^{\frac{1}{2}} .
$$

We do not prove the exact value of the constant in (1-12); see [Merle and Raphaël 2006, Proposition 6]. Finally, we conclude that (1-13) follows from the log-log relationship H1.3, higher-order norm control H2.1, and from $m>0$ small. As an aside, recall that $d s / d t=1 / \lambda^{2}$, so with (5-253) one would conclude

$$
\frac{1}{C}\left|\log \left(T_{\max }-t\right)\right| \leq s(t) \leq C\left|\log \left(T_{\max }-t\right)\right| .
$$

Then from the explicit lower and upper bounds for $b$ in (3-137) and (3-177) we obtain

$$
\frac{1}{C \log \left|\log \left(T_{\max }-t\right)\right|} \leq b(t) \leq \frac{C}{\log \left|\log \left(T_{\max }-t\right)\right|} .
$$

Proof of stable locus of concentration, (1-9). The preliminary estimate (3-133) implies in particular that

$$
\left|\frac{\partial_{s}(r, z)}{\lambda}\right|<1
$$

on $\left[s_{0}, s_{1}\right)$. Then by a change of variable, (5-253) and the bound (3-143) on $T_{\max }$,

$$
\int_{0}^{T_{\max }}\left|\partial_{t}(r, z)\right| d t<\int_{0}^{T_{\max }} \frac{1}{\lambda(t)} d t<\delta\left(\alpha^{*}\right) .
$$

Equation (1-9) follows from choice of initial data C1.1. 
Proof of regularity away from singular ring, (1-11). Given $R>0$, define $\chi_{R}$ to be a suitable modification of $\chi(2-26)$, equal to 1 for $\left|(r, z)-\left(r_{\max }, z_{\max }\right)\right|>R$. Choose some $t(R) \in\left[0, T_{\max }\right)$ such that

$$
A(t) \lambda(t)+\left|(r(t), z(t))-\left(r_{\max }, z_{\max }\right)\right| \ll R \quad \text { for all } t \in\left[t(R), T_{\max }\right),
$$

and hence $\chi_{R} u=\chi_{R} \tilde{u}$ for all $t \in\left[t(R), T_{\max }\right)$. Let $t_{3} \in\left(t(R), T_{\max }\right]$ be the largest value such that

$$
\left\|\chi_{R} u(t)\right\|_{H^{1}}<2\left\|\chi_{R} u(t(R))\right\|_{H^{1}} \quad \text { for all } t \in\left[t(R), t_{3}\right) .
$$

This choice of $t_{3}>t(R)$ is possible since $u(t)$ is strongly continuous in $H^{1}$ at time $t(R)<T_{\max }$. With interpolation, (5-258) replaces the bootstrap hypotheses H2.2 and H2.3. Repeating the arguments of Section 4 proves that $t_{3}=T_{\max }$ and

$$
\|\tilde{u}(t)\|_{H^{1}\left(\left|(r, z)-\left(r_{\max }, z_{\max }\right)\right|>R\right)}<C(R) \quad \text { for all } t \in\left[0, T_{\max }\right) .
$$

This yields (1-11).

Proof of mass concentration, (1-10). Let $R>0$. To begin we will prove there exists a residual profile in $L^{2}$ away from the singular ring:

$$
\tilde{u}(t) \rightarrow u^{*} \quad \text { in } L_{x}^{2}\left(\left|(r, z)-\left(r_{\max }, z_{\max }\right)\right| \geq R\right) \quad \text { as } t \rightarrow T_{\max } .
$$

Then to establish (1-10) we will prove

$$
u^{*} \in L^{2}\left(\mathbb{R}^{N}\right) \text { and } \int\left|u^{*}\right|^{2}=\lim _{t \rightarrow T_{\max }} \int|\tilde{u}(t)|^{2} .
$$

Let $\epsilon_{0}>0$ be arbitrary. Due to (3-178), we may choose $t(R)<T_{\max }$ such that

$$
T_{\max }-t(R)<\frac{\epsilon_{0}}{1+C(R / 4)} \quad \text { and } \quad \int_{t(R)}^{T_{\max }} \int|\nabla \tilde{u}|^{2} d x d t<\epsilon_{0},
$$

where $C(R / 4)$ is the constant from Equation (5-259). We may assume that, for $t \in\left[t(R), T_{\max }\right), u(t)=\tilde{u}$ on $\left\{\left|(r, z)-\left(r_{\max }, z_{\max }\right)\right|>R / 4\right\}$. Let $\tau>0$ be a parameter to be fixed later, and define

$$
v^{\tau}(t, x)=u(t+\tau, x)-u(t, x) .
$$

Since $t(R)<T_{\max }, u(t)$ is strongly continuous in $L^{2}$ at time $t(R)$. Thus, there exists $\tau_{0}$ such that

$$
\int\left|v^{\tau}(t(R))\right|^{2} d x<\epsilon_{0} \quad \text { for all } \tau \in\left[0, \tau_{0}\right] .
$$

Denote a smooth cutoff function $\phi_{R}$ analogous to $\phi_{\infty}$ (see (3-159) and (3-160)):

$$
\phi_{R}(r, z)=\phi_{\infty}^{4}\left(\frac{(r, z)-\left(r_{\max }, z_{\max }\right)}{R}\right) .
$$

By direct calculation,

$$
\frac{1}{2} \partial_{t}\left(\int \phi_{R}\left|v^{\tau}\right|^{2}\right)=\operatorname{Im}\left(\int \nabla \phi_{R} \cdot \nabla v^{\tau} v^{\tau} d x\right)+\operatorname{Im}\left(\int \phi_{R} v^{\tau}\left(\overline{u|u|^{2}(t+\tau)-u|u|^{2}(t)}\right) d x\right) .
$$


Regarding the first term on the right in (5-266), from Hölder and our choice of $t(R)$ we have

$$
\int_{t(R)}^{T_{\max }}\left|\operatorname{Im}\left(\int \nabla \phi_{R} \cdot \nabla v^{\tau} v^{\tau} d x\right) d t\right| \leq C\left(\int_{t(R)}^{T_{\max }} 1^{2} d t\right)^{\frac{1}{2}} \epsilon_{0}^{\frac{1}{2}}<C \epsilon_{0} .
$$

Regarding the second term on the right in (5-266), by homogeneity,

$$
\left|\phi_{R} v^{\tau}\left(\overline{u|u|^{2}(t+\tau)-u|u|^{2}(t)}\right)\right| \leq C\left(\left|\phi_{R}^{\frac{1}{4}} u(t+\tau)\right|^{4}+\left|\phi_{R}^{\frac{1}{4}} u(t)\right|^{4}\right) .
$$

Then, as we did in proving estimate (3-115), apply the Sobolev embedding $H^{\frac{N}{4}} \hookrightarrow L^{4}\left(\mathbb{R}^{N}\right)$ and interpolate, obtaining

$$
\int\left|\phi_{R}^{\frac{1}{4}} u\right|^{4} \leq C\left\|\phi_{R}^{\frac{1}{4}} u\right\|_{H^{\frac{N}{2}-1}}^{2}\left\|\phi_{R}^{\frac{1}{4}} u\right\|_{H^{1}}^{2}
$$

By (5-259), the uniform control of $H^{\frac{N}{2}-1}$, and our choice of $t(R)$,

$$
\int_{t(R)}^{T_{\max }}\left|\phi_{R} v^{\tau}\left(\overline{u|u|^{2}(t+\tau)-u|u|^{2}(t)}\right)\right| d t \leq C \epsilon_{0} .
$$

Through the integration of (5-266) we have proved that

$$
\int \phi_{R}\left|v^{\tau}(t)\right|^{2} d x<C \epsilon_{0} \quad \text { for all } \tau \in\left[0, \tau_{0}\right] \text { and } t \in\left[t(R), T_{\max }-\tau\right) .
$$

This shows that $\tilde{u}$ is Cauchy, which proves (5-260).

We turn to (5-261). Denote the thickness of the toroidal support of the singular profile and radiation by

$$
R(t)=A(t) \lambda(t)
$$

Recall the definition of $A(t)$ in (3-104). By the $\log$-log rate H1.3, we have $A(t) \approx\left|\log \left(T_{\max }-t\right)\right|^{C}$; in particular, $R(t) \rightarrow 0$ with the bound $R(t) \leq\left(T_{\max }-t\right)^{\frac{1}{2}-\delta}$. Now consider

$$
\phi_{R(t), \tau}=\phi_{\infty}^{4}\left(\frac{(r, z)-(r(\tau), z(\tau))}{R(t)}\right),
$$

a family of time-variable cutoffs similar to $\phi_{R(t)}$. For fixed time $t<T_{\max }$ we calculate directly that

$$
\begin{aligned}
& \frac{1}{2} \partial_{\tau}\left(\int \phi_{R(t), \tau}|u(\tau)|^{2} d x\right) \\
& =\frac{1}{R(t)} \operatorname{Im}\left(\int \nabla_{x} \phi_{R(t), \tau} \cdot \nabla_{x} u(\tau) \overline{u(\tau)} d x\right)-\frac{1}{2 R(t)} \int \partial_{\tau}(r(\tau), z(\tau)) \cdot \nabla_{x} \phi_{R(t), \tau}|u(\tau)|^{2} d x,
\end{aligned}
$$

where we use $\nabla_{x} \phi_{R(t), \tau}$ to denote

$$
\left.\nabla_{y} \phi_{\infty}^{4}(y)\right|_{y=\frac{(r, z)-(r(\tau), z(\tau))}{R(t)}}
$$


Regarding the first term on the right in (5-272),

$$
\left|\frac{1}{R(t)} \operatorname{Im}\left(\int \nabla_{x} \phi_{R(t), \tau} \cdot \nabla_{x} u(\tau) \overline{u(\tau)} d x\right)\right| \lesssim \frac{1}{R(t)}\|u(\tau)\|_{H^{1}} \lesssim \frac{1}{A(t) \lambda(t) \lambda(\tau)} .
$$

Regarding the last term of (5-272), apply the preliminary estimate (3-133),

$$
\left.\left.\left|\frac{1}{2 R(t)} \int \partial_{\tau}(r(\tau), z(\tau)) \cdot \nabla_{x} \phi_{R(t), \tau}\right| u(\tau)\right|^{2} d x\left|\lesssim \frac{1}{A(t) \lambda(t) \lambda(\tau)} \int\right| u_{0}\right|^{2} .
$$

Integrate (5-272) in $\tau$, and apply the bounds for $A$ and $\lambda$ to obtain

$$
\begin{aligned}
\mid \int \phi_{R(t), T_{\max }} & \left|u^{*}\right|^{2} d x-\int \phi_{R(t), t}|u(t)|^{2} d x \mid \\
& \leq C \frac{1}{A(t) \lambda(t)} \int_{t}^{T_{\max }} \frac{1}{\lambda(\tau)} d \tau \\
& \leq \frac{C}{\left|\log \left(T_{\max }-t\right)\right|^{C}}\left(\frac{\log \left|\log \left(T_{\max }-t\right)\right|}{T_{\max }-t}\right)^{\frac{1}{2}} \int_{t}^{T_{\max }}\left(\frac{\log \left|\log \left(T_{\max }-\tau\right)\right|}{T_{\max }-\tau}\right)^{\frac{1}{2}} d \tau \\
& \leq \frac{1}{\left|\log \left(T_{\max }-t\right)\right|^{\frac{C}{2}}} .
\end{aligned}
$$

The final inequality relied upon $T_{\max }-t<T_{\max }<\alpha^{*}$, Equation (3-143), both to approximate the integral and then to approximate $C \log \left|\log \left(T_{\max }-t\right)\right|<\left|\log \left(T_{\max }-t\right)\right|^{\frac{C}{2}}$. Taking the $\operatorname{limit} t \rightarrow T_{\max }$ we see that

$$
\int\left|u^{*}\right|^{2}=\lim _{t \rightarrow T_{\max }} \int \phi_{R(t), t}|u(t)|^{2}
$$

From the definition of $A(t)$ and (3-110) we can bound $\int\left(1-\phi_{R(t)}\right)|\tilde{u}(t)|^{2}$ to prove that

$$
\lim _{t \rightarrow T_{\max }} \int \phi_{R(t), t}|u(t)|^{2}=\lim _{t \rightarrow T_{\max }} \int \phi_{R(t), t}|\tilde{u}(t)|^{2}=\lim _{t \rightarrow T_{\max }} \int|\tilde{u}(t)|^{2} ;
$$

this shows that the limit in (5-274) exists and establishes (5-261). This completes the proof of (1-10).

Remark 5.1 (consistency with $u^{*} \notin H^{1}$ ). By repeating the proof of I2.3, we expect that following the proof of (5-260) it could be shown that $\tilde{u}(t) \rightarrow u^{*}$ in $H^{1}\left(\left|(r, z)-\left(r_{\max }, z_{\max }\right)\right| \geq R\right)$. Nevertheless, an attempt to prove a version of (5-261) in $H^{1}$ will fail. Indeed, the last term in (5-272) would require a bound for $|\nabla u(\tau)|$ on the support of $\nabla \phi$, with nothing to take the role of mass conservation.

\section{Appendix}

Proof that $\mathscr{P}$ is nonempty. Choose $r_{0}=1, z_{0}=0, b_{0}>0$ small enough to satisfy (2-29), and $\lambda_{0}$ in the range of $\mathrm{C} 1.3$. Fix some smooth real-valued radially symmetric function $f(y)$, with support in $|y| \leq 2$ and such that $\|f\|_{H^{N}\left(\mathbb{R}^{2}\right)} \leq 1,(f, Q)=1$ and, for any $v \in \mathbb{C}$ to be determined, such that $\epsilon_{0}(y)=v f(y)$ satisfies the orthogonality conditions (2-30). One can explicitly calculate such an $f$ from $\widetilde{Q}_{b_{0}}$. With $\gamma_{0}=0$, we now find $v=v\left(b_{0}\right)$ to satisfy $\mathrm{C} 1.4$ and the small-mass requirement of $\mathrm{C} 1.2$. 
By the choice of $\lambda_{0}$, we have $|(r, z)-(1,0)|<\frac{1}{3}$ on the support of $\widetilde{Q}_{b_{0}}(y)$, which includes the support of $f(y)$. After a change of variables, we will expand $\mu_{\lambda_{0}, 1}(y)$ as $\left(1+\lambda_{0} y_{1}\right)^{N-2}$ so that

$$
\begin{aligned}
\lambda_{0}^{2}\left|E_{0}\right| & =\left.\left|\frac{1}{2} \int\right| \nabla_{y}\left(\widetilde{Q}_{b_{0}}+v f\right)\right|^{2} \mu_{\lambda_{0}, 1}(y) d y-\frac{1}{4} \int\left|\widetilde{Q}_{b_{0}}+v f\right|^{4} \mu_{\lambda_{0}, 1}(y) d y \mid \\
& \left.\lesssim\left|\frac{1}{2} \int\right| \nabla_{y}\left(\widetilde{Q}_{b_{0}}+v f\right)\right|^{2} d y-\frac{1}{4} \int\left|\widetilde{Q}_{b_{0}}+v f\right|^{4} d y \mid+O\left(\lambda_{0}\right),
\end{aligned}
$$

which is a small correction from the two-dimensional energy. Directly from (1-6) we get

$$
\left.\frac{d}{d w}\left(\frac{1}{2} \int\left|\nabla_{y}(Q+w f)\right|^{2} d y-\frac{1}{4} \int|Q+w f|^{4} d y\right)\right|_{w=0}=-\operatorname{Re}(f, Q)=-1
$$

so the left-hand side does not depend on the imaginary component of $v$. By the degenerate energy of $\widetilde{Q}_{b_{0}}$ we may choose the real part of $v$ of the order $|\nu| \leq \Gamma_{b_{0}}^{1-C \eta}$ such that $E_{0}=0$. Note the choice $v=0$ is impossible as the energy of $\widetilde{Q}_{b_{0}}$ alone is too large to satisfy C1.4.

Next we show the momentum requirement of C1.4 is satisfied. Again from the choice of $\lambda_{0}$, the support of $\widetilde{Q}_{b_{0}}+v f$ lies well within $|(r, z)-(1,0)| \leq \frac{1}{2}$, a region where $\nabla_{x} \psi^{(x)}$ is constant; see $(2-34)$. With the radial symmetry of $\widetilde{Q}_{b}$ and $f$ we have

$$
\begin{aligned}
\lambda_{0} \operatorname{Im}\left(\int \nabla_{x} \psi^{(x)} \cdot \nabla_{x} u_{0} \bar{u}_{0}\right) & =(1,1) \cdot \operatorname{Im}\left(\int \nabla_{y}\left(\widetilde{Q}_{b_{0}}+v f\right)\left(\overline{\widetilde{Q}_{b_{0}}+v f}\right) \mu_{\lambda_{0}, 1}(y) d y\right) \\
& =2 \operatorname{Im} \int v f \overline{\widetilde{Q}_{b_{0}}} d y+\mathcal{O}\left(\lambda_{0}\right),
\end{aligned}
$$

and there is a $O\left(\lambda_{0}\right)$ choice of the imaginary part of $v$ such that (5-277) is zero. Finally, we note that C1.4 is satisfied,

$$
\left\|\tilde{u}_{0}\right\|_{L^{2}\left(\mathbb{R}^{N}\right)}=|v|\left(\int|f(y)|^{2} \mu_{\lambda_{0}, 1}(y) d y\right)^{\frac{1}{2}}<\alpha^{*} .
$$

The requirements $\mathrm{C} 2.2$ and $\mathrm{C} 2.3$ are automatic from the support of $f$. The constant $C$ in $\mathrm{C} 2.1$ is due to Lemma 2.2 and the choice of $v$.

Relationship with the classic virial argument. For data $u_{0} \in H^{1}$ with finite variance, due to the classic virial identity, a sufficient condition for blowup is

$$
\left[\operatorname{Im}\left(\int x \nabla u_{0} \overline{u_{0}}\right)\right]^{2}>2\left\|x u_{0}\right\|_{L^{2}}^{2} E\left(u_{0}\right)
$$

We remark that there exists $u_{0} \in \mathscr{P}$ for which condition (5-279) fails.

\section{Acknowledgements}

The author would like to thank Pierre Raphaël and Jérémie Szeftel for correspondence and for sharing the draft of their work [Raphaël and Szeftel 2009], Justin Holmer and Svetlana Roudenko for conversations, and the referee for helpful suggestions. The author is also indebted to his advisor, Jim Colliander, for his attention and guidance. This work is part of the author's Ph.D. thesis at the University of Toronto. 


\section{References}

[Berestycki and Lions 1983] H. Berestycki and P.-L. Lions, "Nonlinear scalar field equations. I. Existence of a ground state", Arch. Rational Mech. Anal. 82:4 (1983), 313-345. MR 84h:35054a

[Cazenave 2003] T. Cazenave, Semilinear Schrödinger equations, Courant Lecture Notes in Mathematics 10, CIMS, New York, 2003. MR 2004j:35266 Zbl 1055.35003

[Coffman 1972] C. V. Coffman, "Uniqueness of the ground state solution for $\Delta u-u+u^{3}=0$ and a variational characterization of other solutions", Arch. Rational Mech. Anal. 46 (1972), 81-95. MR 48 \#11814 Zbl 0249.35029

[Duyckaerts and Merle 2009] T. Duyckaerts and F. Merle, "Dynamic of threshold solutions for energy-critical NLS", Geom. Funct. Anal. 18:6 (2009), 1787-1840. MR 2010e:35258 Zbl 05603849

[Duyckaerts and Roudenko 2010] T. Duyckaerts and S. Roudenko, "Threshold solutions for the focusing 3D cubic Schrödinger equation", Rev. Mat. Iberoam. 26:1 (2010), 1-56. MR 2011c:35533 Zbl 1195.35276

[Duyckaerts et al. 2008] T. Duyckaerts, J. Holmer, and S. Roudenko, "Scattering for the non-radial 3D cubic nonlinear Schrödinger equation”, Math. Res. Lett. 15:6 (2008), 1233-1250. MR 2010e:35257

[Fibich et al. 2006] G. Fibich, F. Merle, and P. Raphaël, "Proof of a spectral property related to the singularity formation for the $L^{2}$ critical nonlinear Schrödinger equation”, Phys. D 220:1 (2006), 1-13. MR 2007d:35254 Zbl 1100.35097

[Fibich et al. 2007] G. Fibich, N. Gavish, and X.-P. Wang, "Singular ring solutions of critical and supercritical nonlinear Schrödinger equations”, Phys. D 231:1 (2007), 55-86. MR 2008k:35440 Zbl 1118.35043

[Holmer and Roudenko 2007] J. Holmer and S. Roudenko, "On blow-up solutions to the 3D cubic nonlinear Schrödinger equation”, Appl. Math. Res. Express. AMRX 2007:1 (2007), Art. ID abm004. MR 2008i:35227

[Holmer and Roudenko 2008] J. Holmer and S. Roudenko, "A sharp condition for scattering of the radial 3D cubic nonlinear Schrödinger equation”, Comm. Math. Phys. 282:2 (2008), 435-467. MR 2009h:35403 Zbl 1155.35094

[Holmer and Roudenko 2010] J. Holmer and S. Roudenko, "Divergence of infinite-variance nonradial solutions to the 3D NLS equation”, Comm. Partial Differential Equations 35:5 (2010), 878-905. MR 2011m:35353 Zbl 1195.35277

[Holmer and Roudenko 2011] J. Holmer and S. Roudenko, "A class of solutions to the 3D cubic nonlinear Schrödinger equation that blows up on a circle”, Appl. Math. Res. Express. AMRX 2011:1 (2011), 23-94. MR 2012b:35323 Zbl 1220.35159

[Holmer et al. 2010] J. Holmer, R. Platte, and S. Roudenko, "Blow-up criteria for the 3D cubic nonlinear Schrödinger equation", Nonlinearity 23:4 (2010), 977-1030. MR 2011c:35538 Zbl 1202.37105

[Kenig and Merle 2006] C. E. Kenig and F. Merle, "Global well-posedness, scattering and blow-up for the energy-critical, focusing, non-linear Schrödinger equation in the radial case", Invent. Math. 166:3 (2006), 645-675. MR 2007g:35232

[Killip et al. 2009] R. Killip, T. Tao, and M. Visan, "The cubic nonlinear Schrödinger equation in two dimensions with radial data”, J. Eur. Math. Soc. (JEMS) 11:6 (2009), 1203-1258. MR 2010m:35487 Zbl 1187.35237

[Kwong 1989] M. K. Kwong, "Uniqueness of positive solutions of $\Delta u-u+u p=0$ in $\mathbf{R}^{n}$ ", Arch. Rational Mech. Anal. 105:3 (1989), 243-266. MR 90d:35015 Zbl 0676.35032

[Landman et al. 1988] M. J. Landman, G. C. Papanicolaou, C. Sulem, and P.-L. Sulem, "Rate of blowup for solutions of the nonlinear Schrödinger equation at critical dimension", Phys. Rev. A (3) 38:8 (1988), 3837-3843. MR 89k:35218

[Lions 1982] P.-L. Lions, "Symétrie et compacité dans les espaces de Sobolev", J. Funct. Anal. 49:3 (1982), 315-334. MR 84k:46027 Zbl 0501.46032

[Mariş 2002] M. Mariş, "Existence of nonstationary bubbles in higher dimensions", J. Math. Pures Appl. (9) 81:12 (2002), 1207-1239. MR 2004e:35213 Zbl 1040.35116

[Martel and Merle 2001] Y. Martel and F. Merle, "Instability of solitons for the critical generalized Korteweg-de Vries equation”, Geom. Funct. Anal. 11:1 (2001), 74-123. MR 2002g:35182 Zbl 0985.35071

[McLeod 1993] K. McLeod, "Uniqueness of positive radial solutions of $\Delta u+f(u)=0$ in $\mathbf{R}^{n}$. II", Trans. Amer. Math. Soc. 339:2 (1993), 495-505. MR 94b:35105 Zbl 0804.35034

[Merle 1993] F. Merle, "Determination of blow-up solutions with minimal mass for nonlinear Schrödinger equations with critical power", Duke Math. J. 69:2 (1993), 427-454. MR 94b:35262 Zbl 0808.35141 
[Merle and Raphaël 2003] F. Merle and P. Raphael, "Sharp upper bound on the blow-up rate for the critical nonlinear Schrödinger equation", Geom. Funct. Anal. 13:3 (2003), 591-642. MR 2005j:35207 Zbl 1061.35135

[Merle and Raphaël 2004] F. Merle and P. Raphael, "On universality of blow-up profile for $L^{2}$ critical nonlinear Schrödinger equation”, Invent. Math. 156:3 (2004), 565-672. MR 2006a:35283 Zbl 1067.35110

[Merle and Raphaël 2005a] F. Merle and P. Raphael, "The blow-up dynamic and upper bound on the blow-up rate for critical nonlinear Schrödinger equation”, Ann. of Math. (2) 161:1 (2005), 157-222. MR 2006k:35277 Zbl 1185.35263

[Merle and Raphaël 2005b] F. Merle and P. Raphael, "Profiles and quantization of the blow up mass for critical nonlinear Schrödinger equation”, Comm. Math. Phys. 253:3 (2005), 675-704. MR 2006m:35346 Zbl 1062.35137

[Merle and Raphä̈l 2006] F. Merle and P. Raphael, "On a sharp lower bound on the blow-up rate for the $L^{2}$ critical nonlinear Schrödinger equation”, J. Amer. Math. Soc. 19:1 (2006), 37-90. MR 2006j:35223 Zbl 1075.35077

[Nakanishi and Schlag 2010] K. Nakanishi and W. Schlag, "Global dynamics above the ground state energy for the cubic NLS equation in 3D", preprint, 2010. arXiv 1007.4025

[Ogawa and Tsutsumi 1991] T. Ogawa and Y. Tsutsumi, "Blow-up of $H^{1}$ solution for the nonlinear Schrödinger equation", $J$. Differential Equations 92:2 (1991), 317-330. MR 92k:35262 Zbl 0739.35093

[Perelman 2001] G. Perelman, "On the formation of singularities in solutions of the critical nonlinear Schrödinger equation", Ann. Henri Poincaré 2:4 (2001), 605-673. MR 2002m:35205 Zbl 1007.35087

[Persson 1964] A. Persson, "Compact linear mappings between interpolation spaces", Ark. Mat. 5 (1964), 215-219. MR 29 \#3871 Zbl 0128.35204

[Raphäl 2005] P. Raphael, "Stability of the log-log bound for blow up solutions to the critical non linear Schrödinger equation”, Math. Ann. 331:3 (2005), 577-609. MR 2006b:35303

[Raphäl 2006] P. Raphaël, "Existence and stability of a solution blowing up on a sphere for an $L^{2}$-supercritical nonlinear Schrödinger equation”, Duke Math. J. 134:2 (2006), 199-258. MR 2007k:35472 Zbl 1117.35077

[Raphä̈l and Szeftel 2009] P. Raphäl and J. Szeftel, "Standing ring blow up solutions to the $N$-dimensional quintic nonlinear Schrödinger equation”, Comm. Math. Phys. 290:3 (2009), 973-996. MR 2010k:35469 Zbl 1184.35295

[Sulem and Sulem 1999] C. Sulem and P.-L. Sulem, The nonlinear Schrödinger equation: self-focusing and wave collapse, Applied Mathematical Sciences 139, Springer, New York, 1999. MR 2000f:35139 Zbl 0928.35157

[Tao 2006] T. Tao, Nonlinear dispersive equations, CBMS Regional Conference Series in Mathematics: local and global analysis 106, Amer. Math. Soc., Providence, RI, 2006. MR 2008i:35211 Zbl 1106.35001

[Weinstein 1983] M. I. Weinstein, "Nonlinear Schrödinger equations and sharp interpolation estimates", Comm. Math. Phys. 87:4 (1983), 567-576. MR 84d:35140 Zbl 0527.35023

[Weinstein 1985] M. I. Weinstein, "Modulational stability of ground states of nonlinear Schrödinger equations", SIAM J. Math. Anal. 16:3 (1985), 472-491. MR 86i:35130 Zbl 0583.35028

Received 5 Feb 2010. Revised 27 Sep 2010. Accepted 14 Nov 2010.

IAN ZWIERS: zwiers@math.ubc.ca

Department of Mathematics, University of British Columbia, Vancouver, V6T 1Z2, Canada 


\title{
Analysis \& PDE
}

\author{
msp.berkeley.edu/apde
}

EDITORS

EDITOR-IN-CHIEF

Maciej Zworski

University of California

Berkeley, USA

BOARD OF EDITORS

\begin{tabular}{|c|c|c|c|}
\hline Michael Aizenman & $\begin{array}{l}\text { Princeton University, USA } \\
\text { aizenman@math.princeton.edu }\end{array}$ & Nicolas Burq & $\begin{array}{l}\text { Université Paris-Sud 11, France } \\
\text { nicolas.burq@math.u-psud.fr }\end{array}$ \\
\hline Luis A. Caffarelli & $\begin{array}{l}\text { University of Texas, USA } \\
\text { caffarel@ math.utexas.edu }\end{array}$ & un-Yung Alice Chang & $\begin{array}{l}\text { Princeton University, USA } \\
\text { chang@ math.princeton.edu }\end{array}$ \\
\hline Michael Christ & $\begin{array}{l}\text { University of California, Berkeley, USA } \\
\text { mchrist@math.berkeley.edu }\end{array}$ & Charles Fefferman & $\begin{array}{l}\text { Princeton University, USA } \\
\text { cf@math.princeton.edu }\end{array}$ \\
\hline Ursula Hamenstaedt & $\begin{array}{l}\text { Universität Bonn, Germany } \\
\text { ursula@math.uni-bonn.de }\end{array}$ & Nigel Higson & $\begin{array}{l}\text { Pennsylvania State Univesity, USA } \\
\text { higson@math.psu.edu }\end{array}$ \\
\hline Vaughan Jones & $\begin{array}{l}\text { University of California, Berkeley, USA } \\
\text { vfr@math.berkeley.edu }\end{array}$ & Herbert Koch & $\begin{array}{l}\text { Universität Bonn, Germany } \\
\text { koch@math.uni-bonn.de }\end{array}$ \\
\hline Izabella Laba & $\begin{array}{l}\text { University of British Columbia, Canada } \\
\text { ilaba@math.ubc.ca }\end{array}$ & Gilles Lebeau & $\begin{array}{l}\text { Université de Nice Sophia Antipolis, France } \\
\text { lebeau@unice.fr }\end{array}$ \\
\hline László Lempert & $\begin{array}{l}\text { Purdue University, USA } \\
\text { lempert@math.purdue.edu }\end{array}$ & Richard B. Melrose & $\begin{array}{l}\text { Massachussets Institute of Technology, USA } \\
\text { rbm@math.mit.edu }\end{array}$ \\
\hline Frank Merle & $\begin{array}{l}\text { Université de Cergy-Pontoise, France } \\
\text { Frank.Merle@u-cergy.fr }\end{array}$ & William Minicozzi II & $\begin{array}{l}\text { Johns Hopkins University, USA } \\
\text { minicozz@ math.jhu.edu }\end{array}$ \\
\hline Werner Müller & $\begin{array}{l}\text { Universität Bonn, Germany } \\
\text { mueller@math.uni-bonn.de }\end{array}$ & Yuval Peres & $\begin{array}{l}\text { University of California, Berkeley, USA } \\
\text { peres@stat.berkeley.edu }\end{array}$ \\
\hline Gilles Pisier & $\begin{array}{l}\text { Texas A\&M University, and Paris } 6 \\
\text { pisier@math.tamu.edu }\end{array}$ & Tristan Rivière & $\begin{array}{l}\text { ETH, Switzerland } \\
\text { riviere@math.ethz.ch }\end{array}$ \\
\hline Igor Rodnianski & $\begin{array}{l}\text { Princeton University, USA } \\
\text { irod@math.princeton.edu }\end{array}$ & Wilhelm Schlag & $\begin{array}{l}\text { University of Chicago, USA } \\
\text { schlag@math.uchicago.edu }\end{array}$ \\
\hline Sylvia Serfaty & $\begin{array}{l}\text { New York University, USA } \\
\text { serfaty@ cims.nyu.edu }\end{array}$ & Yum-Tong Siu & $\begin{array}{l}\text { Harvard University, USA } \\
\text { siu@math.harvard.edu }\end{array}$ \\
\hline Terence Tao & $\begin{array}{l}\text { University of California, Los Angeles, USA } \\
\text { tao@math.ucla.edu }\end{array}$ & Michael E. Taylor & $\begin{array}{l}\text { Univ. of North Carolina, Chapel Hill, USA } \\
\text { met@math.unc.edu }\end{array}$ \\
\hline Gunther Uhlmann & $\begin{array}{l}\text { University of Washington, USA } \\
\text { gunther@math.washington.edu }\end{array}$ & András Vasy & $\begin{array}{l}\text { Stanford University, USA } \\
\text { andras@math.stanford.edu }\end{array}$ \\
\hline an Virgil Voiculescu & $\begin{array}{l}\text { University of California, Berkeley, USA } \\
\text { dvv@ math.berkeley.edu }\end{array}$ & Steven Zelditch & $\begin{array}{l}\text { Northwestern University, USA } \\
\text { zelditch@math.northwestern.edu }\end{array}$ \\
\hline
\end{tabular}

\section{PRODUCTION}

contact@msp.org

Silvio Levy, Scientific Editor

Sheila Newbery, Senior Production Editor

See inside back cover or msp.berkeley.edu/apde for submission instructions.

The subscription price for 2011 is US \$120/year for the electronic version, and \$180/year for print and electronic. Subscriptions, requests for back issues from the last three years and changes of subscribers address should be sent to Mathematical Sciences Publishers, Department of Mathematics, University of California, Berkeley, CA 94720-3840, USA.

Analysis \& PDE, at Mathematical Sciences Publishers, Department of Mathematics, University of California, Berkeley, CA 94720-3840 is published continuously online. Periodical rate postage paid at Berkeley, CA 94704, and additional mailing offices.

APDE peer review and production are managed by EditFLOW ${ }^{\mathrm{TM}}$ from Mathematical Sciences Publishers.

PUBLISHED BY

mathematical sciences publishers

http://msp.org/

A NON-PROFIT CORPORATION

Typeset in IATEX

Copyright $(2011$ by Mathematical Sciences Publishers 


\section{ANALYSIS \& PDE}

\section{Volume $4 \quad$ No. $5 \quad 2011$}

Periodic solutions of nonlinear Schrödinger equations: a paradifferential approach JEAN-MARC DELORT

Standing ring blowup solutions for cubic nonlinear Schrödinger equations IAN ZWIERS

Non-Weyl resonance asymptotics for quantum graphs

E. BRIAN DAVIES and AlEXANDER Pushnitski

Improved lower bounds for Ginzburg-Landau energies via mass displacement 